\title{
Air-Coupled Impact-Echo Damage Detection in Reinforced Concrete Using Wavelet Transforms
}

\author{
By \\ Tyler Aaron Epp
}

A Thesis submitted to the Faculty of Graduate Studies of

The University of Manitoba

in partial fulfillment of the requirements of the degree of

\section{Master of Science}

Department of Civil Engineering

Faculty of Engineering

University of Manitoba

Winnipeg

Copyright (C 2017 by Tyler Aaron Epp 


\begin{abstract}
Decline of the state of infrastructure in North America due to aging over the past decades has spawned burgeoning interest in the detection of internal damage of reinforced concrete (RC) structures via nondestructive testing. This study proposes a new impact-echo analysis method using wavelet transforms. The signals recorded from the microphones are analyzed using percentage of energy information to detect in-situ damages. Further, an artificial neural network (ANN) was used in order to test the feasibility of increasing the automaticity of the impact-echo method and a semi-autonomous sensing setup was used to the same end. The proposed wavelet transform-based approach showed improved accuracy when covering broader areas over conventional methods. The use of an ANN removed the need for a user-defined cutoff value for the classification of intact and damaged locations when a least-squared distance approach was used. It is postulated that this may contribute significantly to testing time reduction.
\end{abstract}




\section{ACKNOWLEDGEMENTS}

I would first like to thank my advisor Dr. Young-Jin Cha, Assistant Professor of Structural Engineering in the Department of Civil Engineering at the University of Manitoba, for his continuous support and guidance. I would also like to thank him for the knowledge he has instilled in me and for setting an example of curiosity, determination, and confidence.

My appreciation also goes to my committee members, Dr. Dagmar Svecova and Dr. Nan Wu for their thoughtful insight, support, and encouragement throughout this endeavour.

Thanks also to my colleagues for their help and conversations. This work is stronger for your efforts and input.

The financial support from the Province of Manitoba through the Manitoba Graduate Scholarship and from JMBT and Vector through the JMBT - Vector Scholarship for Research in Civionics Engineering is gratefully acknowledged.

I would also like to thank Dr. Chad Klowak, PEng, Mr. Brendan Pachal, and Mr. Grant Whiteside, the technical staff at the McQuade Structural Laboratory at the University of Manitoba, for their technical assistance and advice.

Finally, I would like to acknowledge my family and friends for their unconditional love and support. In particular, I would like to thank my parents and girlfriend, Alex Cornick, without whom none of this would have been possible. 


\section{Table of Contents}

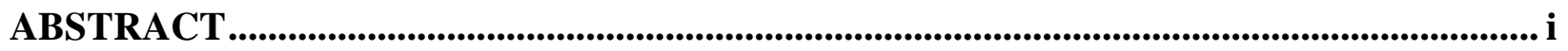

ACKNOWLEDGEMENTS ....................................................................................................

List of Tables .............................................................................................................................. vi

List of Figures..................................................................................................................................................... vii

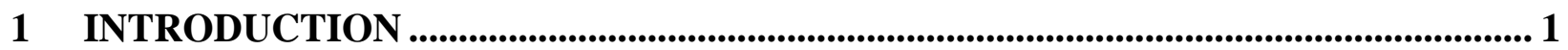

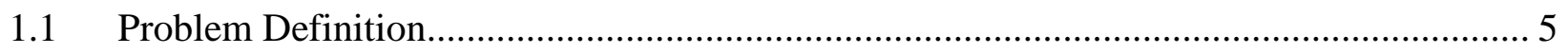

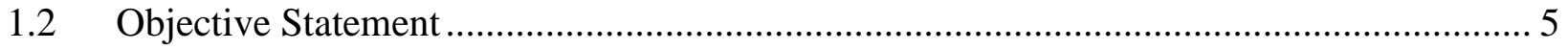

2 LITERATURE REVIEW ........................................................................................... 6

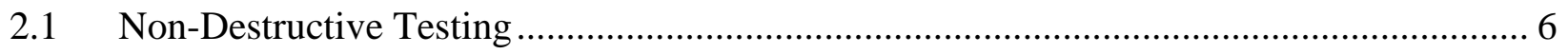

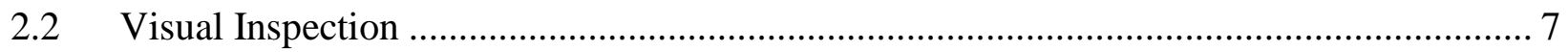

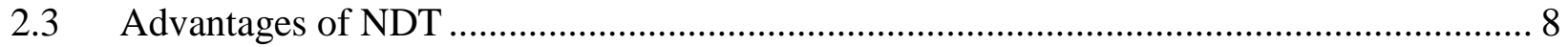

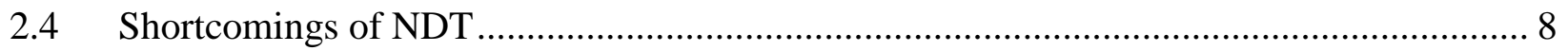

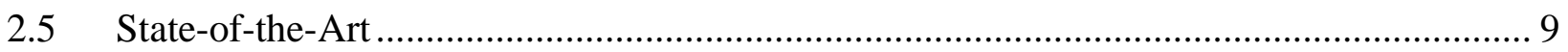

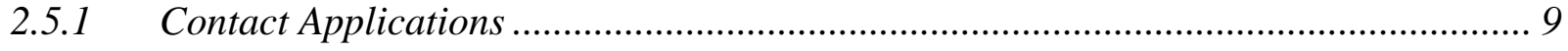

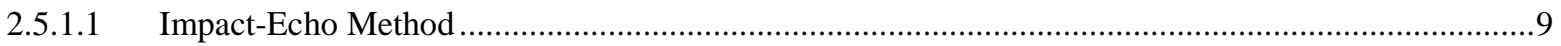

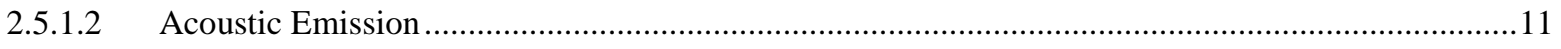

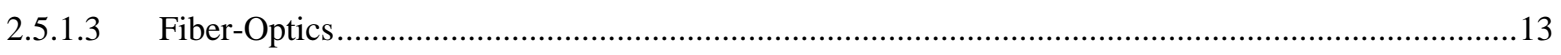

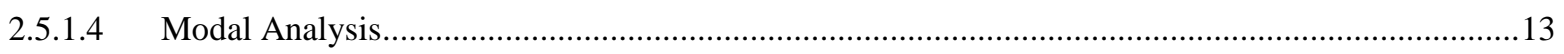

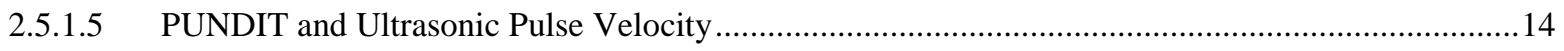

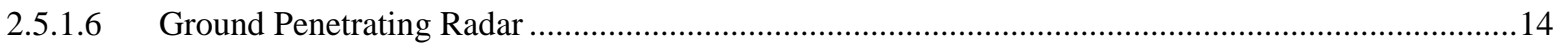

2.5.2 Non-Contact Applications ................................................................................. 16

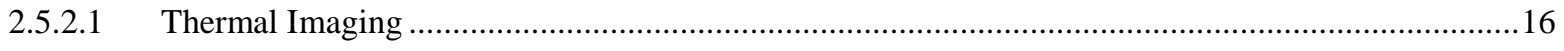

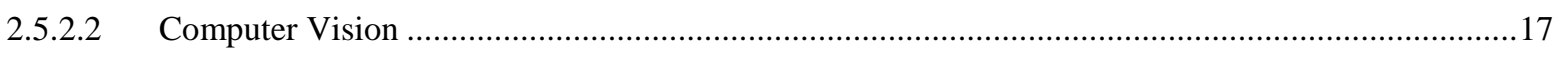

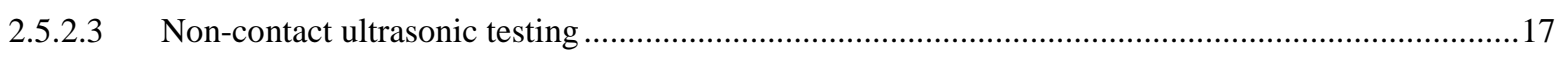

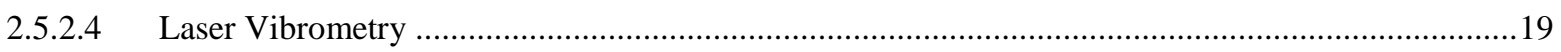

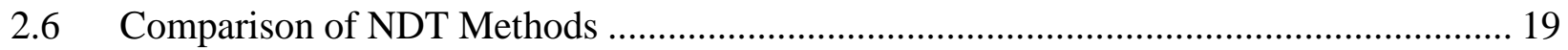

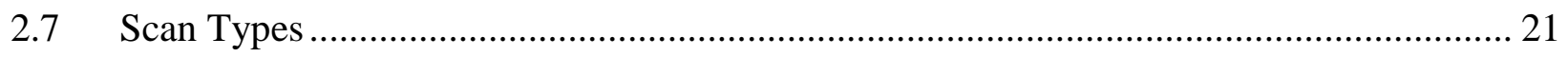

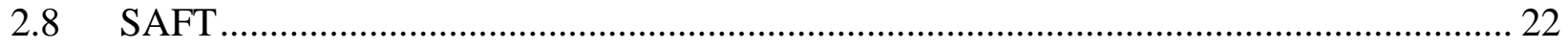

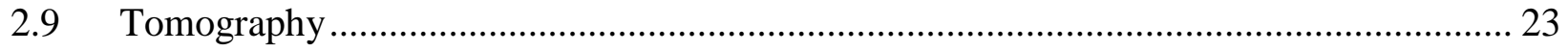




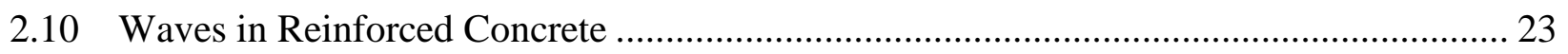

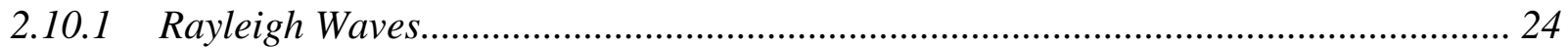

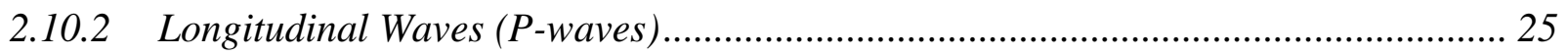

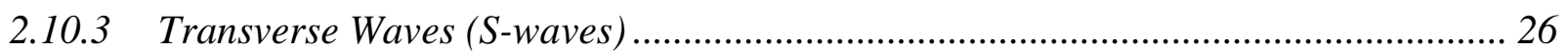

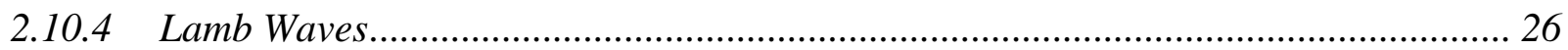

2.10.5 Snell's Law and the Second Critical Angle ............................................................. 27

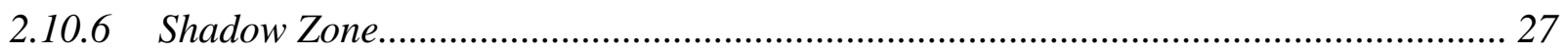

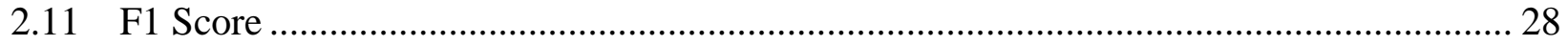

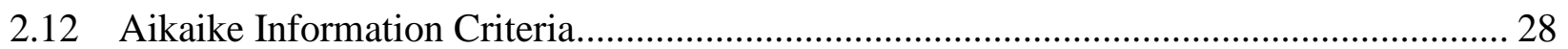

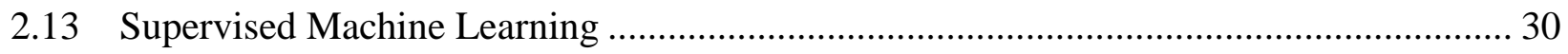

3 IMPACT-ECHO DAMAGE DETECTION......................................................................... 31

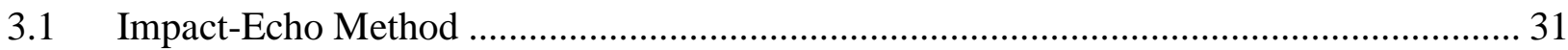

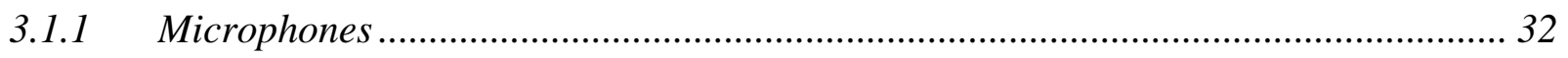

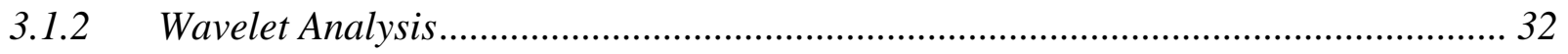

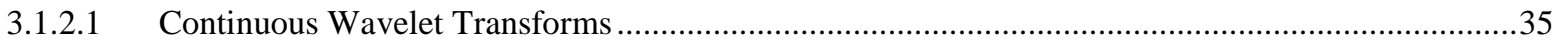

3.1.2.2 Discrete Wavelet Transforms ..................................................................................................

3.1.2.3 Wavelet Scaling.................................................................................................................. 40

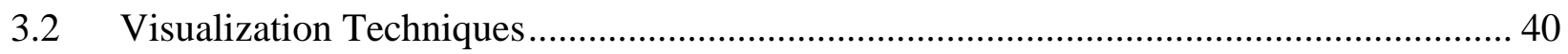

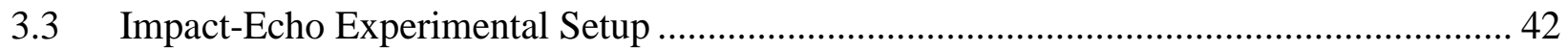

$3.4 \quad$ Impact-Echo Testing Results and Discussion................................................................. 47

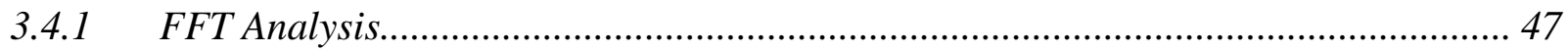

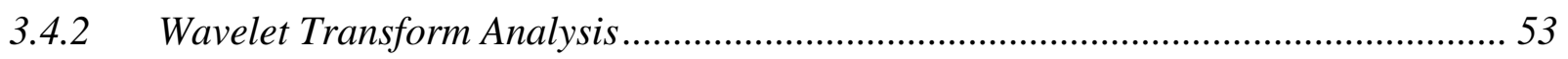

3.4.2.1 Relative Energy of Coefficients Produced from Wavelet Transforms ….........................................55

3.5 Comparison of Performance Using FFT and Wavelet Transforms ……………............... 63

4 FIELD TESTING OF IMPACT-ECHO METHOD ............................................................ 67

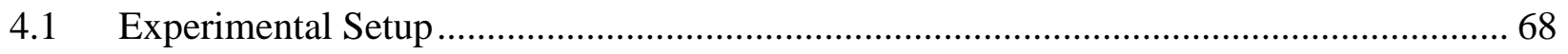

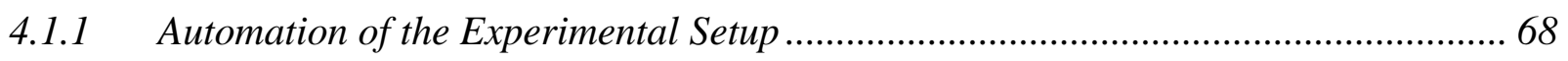

4.1.2 Condition of the Structure at Testing ……………….............................................. 70

4.2 Results and Discussion ............................................................................................. 71

5 ARTIFICIAL NEURAL NETWORK ANALYSIS............................................................. 75

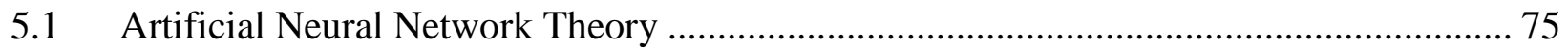




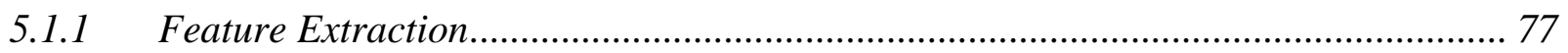

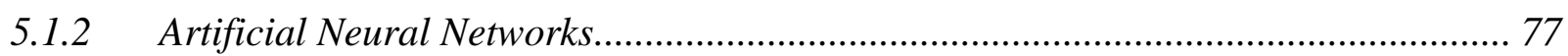

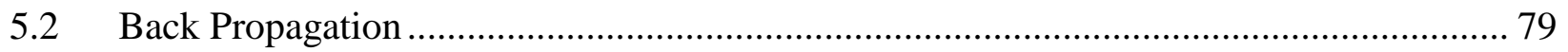

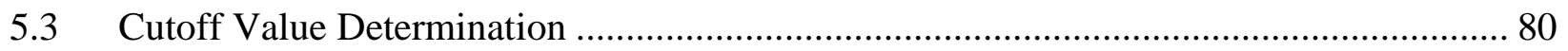

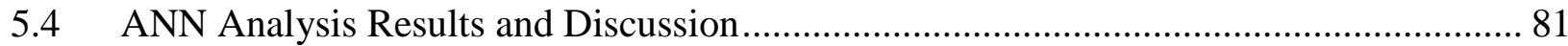

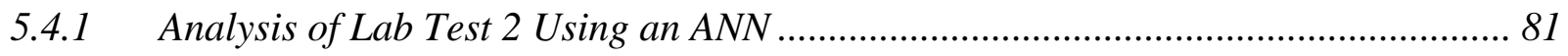

5.4.2 Analysis of Parking Garage Beam B2 Using an ANN........................................... 84

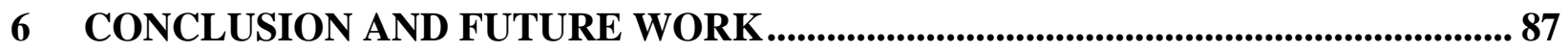

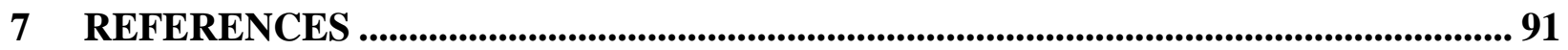

APPENDIX A: WAVELET COEFFICIENT CALCULATIONS ............................................. 1 


\section{List of Tables}

Table 1: Comparison of advantages and disadvantages of NDT methods .............................. 20

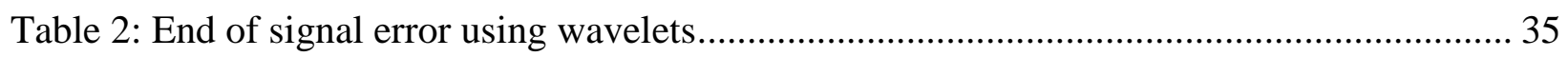

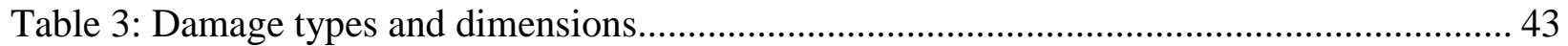

Table 4: Scale and cutoff range for EIF using wavelet transforms Test 1............................. 61

Table 5: Scale and cutoff range for EIF using wavelet transforms Test 2 ............................. 63

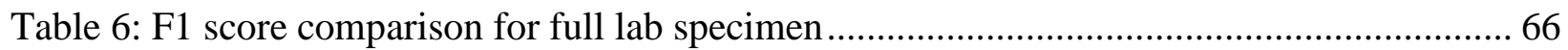

Table 7: F1 score comparison for beam B2 …............................................................ 74

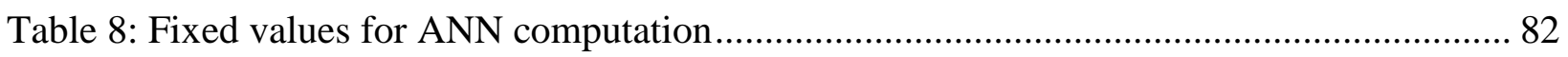

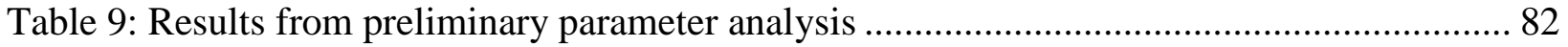

Table 10: Results from preliminary parameter analysis from 13000 data points ...................... 85

Table 11: Continuous wavelet transforms coefficient calculations using linear integration .......... 2

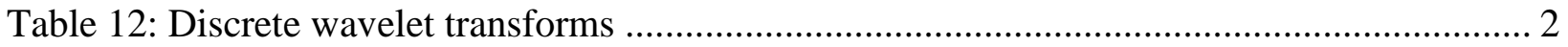




\section{List of Figures}

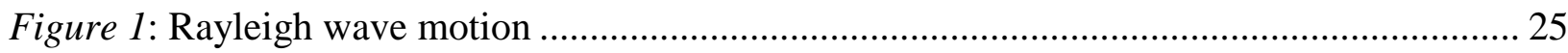

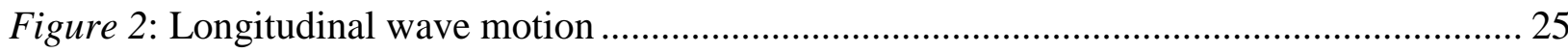

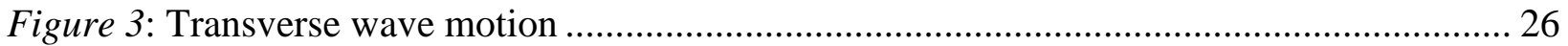

Figure 4: AIC from Impact-Echo Test 2 a) Point 4 (Intact) and b) Point 124 (Damaged) ......... 29

Figure 5: Wavelets used for wavelet analysis (a) Morlet Wavelet (b) DB 4 Wavelet (c) DB 6

Wavelet (d) DB 7 Wavelet (e) Mexican Hat Wavelet...................................................... 33

Figure 6: Implementing continuous wavelet transforms for arbitrary scale ............................. 36

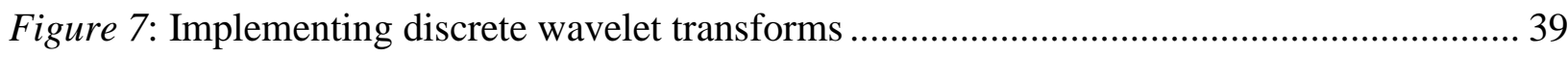

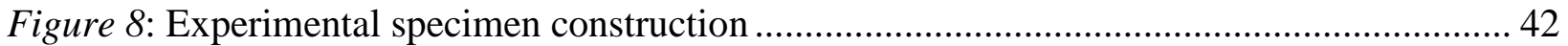

Figure 9: (a) Simply supported $4000 \times 360 \times 300 \mathrm{~mm}$ specimen and (b) Plan view (top) and elevation view (bottom) of the damages and damage locations in the reinforced concrete

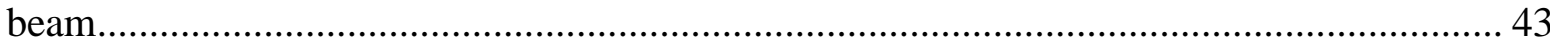

Figure 10: Example of microphone setup and impact location ............................................. 45

Figure 11: Regions of testing not accessible to sensors for (a) Test 1 and (b) Test $2 \ldots \ldots \ldots \ldots \ldots . . . .47$

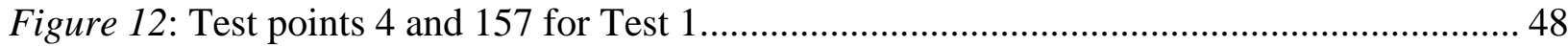

Figure 13: FFT for intact and damaged points of Test 1 .................................................... 49

Figure 14: Spectral comparison for test point 4 and 157 from Test 1 .................................... 49

Figure 15: Imaging of damage using FFT for Test 1 data (MIC 1 cutoff value - MIC 2 cutoff

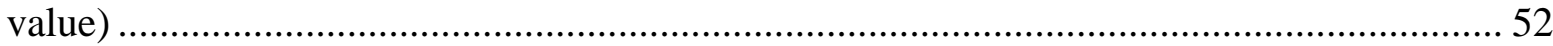

Figure 16: Imaging of damage using FFT and cutoff criteria for Test 2 (MIC 1 cutoff - MIC 2

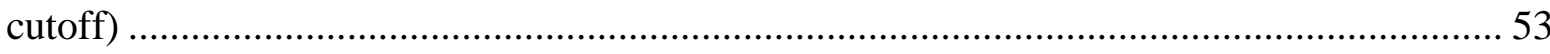

Figure 17: Corresponding frequency and scales for the Morlet wavelet................................. 54

Figure 18: Corresponding frequency and scales of wavelets .............................................. 54

Figure 19: Morlet wavelet transform for Point 157 MIC 1 Test 1 ........................................ 55

Figure 20: DB 4 (left), Morlet (center), and Mexican Hat (right) wavelet scalogram for scales 0.5:0.5:500 56

Figure 21: Focused view of DB4 wavelet with a scale range of 0.5:0.5:40 for MIC 2 point 4

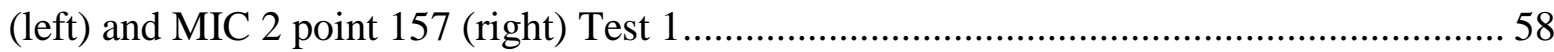

Figure 22: Flow chart of EIF implementation to visualize internal structural state 59 
Figure 23: Imaging of damage using EIF from wavelet transforms for Test 1 ...................... 61

Figure 24: Imaging of damage using EIF from wavelet transforms for Test 2 ....................... 62

Figure 25: Comparison of performance between FFT and Mexican Hat wavelet analysis for (a)

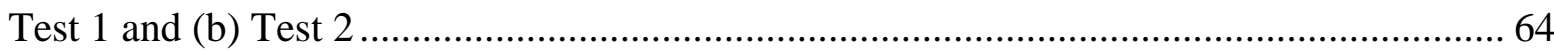

Figure 26: Comparison of performance between FFT and DB4 wavelet analysis for (a) Test 1 and (b) Test 2

Figure 27: Comparison of performance between FFT and DB6 wavelet analysis for (a) Test 1 and (b) Test 2 65

Figure 28: Comparison of performance between FFT and DB7 wavelet analysis for (a) Test 1 and (b) Test 2 .....

Figure 29: Comparison of performance between FFT and Morlet wavelet analysis for (a) Test 1 and (b) Test 2 66

Figure 30: Parking garage test setup (a) bottom view (b) side view ...................................... 68

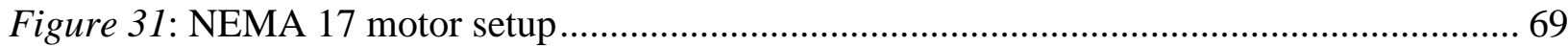

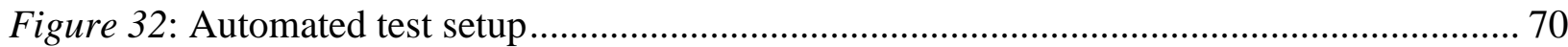

Figure 33: Condition of beam (a) comprehensive view and (b) compilation of test area images

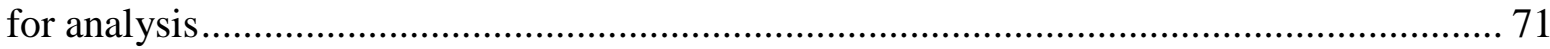

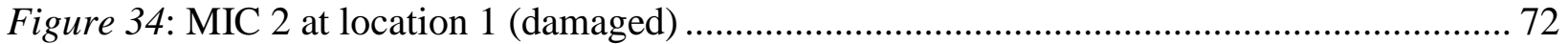

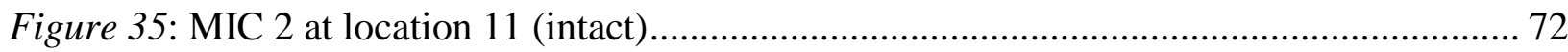

Figure 36: Percentage of energy comparison of MIC 2 at location 1 (damaged) (a) and 11 (intact) (b) 73

Figure 37: Parking garage delamination and cracking using Mexican Hat wavelet with cutoff of 0.12 74

Figure 38: Parking garage delamination and cracking using Mexican Hat wavelet with cutoff of 0.15 74

Figure 39: Parking garage delamination and cracking using Mexican Hat wavelet with cutoff of 0.18 74

Figure 40: Schematic view of semi-automated damage detection using air-coupled impact-echo

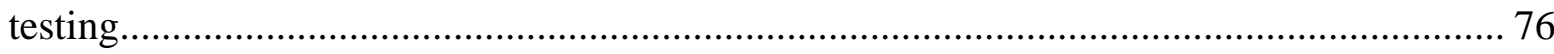

Figure 41: Back propagation algorithm for output and hidden layer ..................................... 79

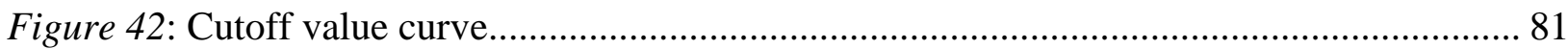


Figure 43: Damage detection with 10 training points, 40 hidden layer neurons, and 1000

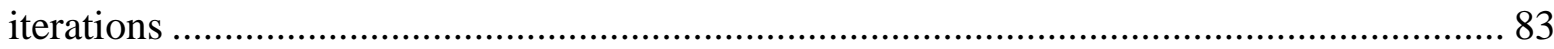

Figure 44: Damage detection with solely EIF and automated cutoff value ........................... 83

Figure 45: Damage detection with 34 training points, 40 hidden layer neurons, and 1000 ........ 84

Figure 46: Damage detection with 10 training points, 20 hidden layer neurons, and $100 \ldots \ldots \ldots . . .85$

Figure 47: Damage detection with solely EIF and automated cutoff value ............................. 86

Figure 48: Comparison between damaged area of the beam (a) and the results of the damage

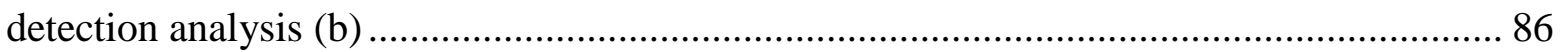

Figure 49: Damage detection with 34 training points, 40 hidden layer neurons, and $100 \ldots \ldots \ldots . .86$ 


\section{INTRODUCTION}

Infrastructure in North America, including bridges, is in dire need of upgrading with the average Canadian bridge exceeding $49 \%$ of its design life and the average American bridge exceeding $56 \%$ of its design life based on a 75-year design life $[69,70]$. The impending maintenance and replacement costs of these structures are estimated in the trillions of dollars in the coming years [70]. Addressing the current state of infrastructure in Canada and the U.S. is important because of the scale of its deterioration, the cost to repair and replace infrastructure, the reliance on infrastructure by the public and industry, and the public safety concerns inherent with aging infrastructure. Structural health monitoring (SHM) has become multi-facetted in response to the complexity of the problem, with numerous approaches available to investigate the different problems. Infrastructure is currently facing many systemic issues, but it is also an area of great improvement and innovation. Some of the infrastructure in Canada and the U.S. is part of the post-World War II economic boom that took place. The increased production over this period has led to a large number of bridges and roadways that are currently reaching the end of their life expectancy. Overseeing bodies must therefore determine where measures must be taken to improve infrastructure and how to best use the limited resources available in doing so.

One approach in solving the problem of aging infrastructure and public safety is improving the way infrastructure is monitored; namely, non-destructive testing (NDT). NDT allows structures to be analyzed without damaging the structure. Many methods of NDT exist, including but not limited to various scanning techniques, visual inspection, chain dragging, fiberoptic sensors [51], computer vision $[31,32,35,71,72]$, acoustic emission sensors [44, 60, 64, 66, 81], and air-coupled and contact impact-echo techniques [5, 27, 39, 41, 46, 47, 49, 62, 77]. 
Detection of internal damage in concrete structures is of particular interest because traditional visual inspection falls short of being able to assess for internal damage in many instances and internal damage is difficult to detect even when using alternative testing methods. Recent research has therefore been focused on identifying several types of internal damage in RC structures. Among these damages are breathing cracks [1, 2], micro cracking [3], delamination [4], and voids and inclusions [13]. Voids may be present from conception of new structures if the workability of the concrete is poor or the concrete is not vibrated properly, leading to air pockets in the structure. Delamination occurs when steel reinforcement corrodes, thus expanding and creating a horizontal crack in the structure running along the plane of the reinforcement and concrete cover. Alternatively, corrosion of reinforcement can cause vertical cracking through the concrete that radiates from the reinforcement. Cracking can also occur because of thermal effects of changing temperature on the structure and shrinking during curing. Cracks that propagate to the surface can be found by visual inspection, although depth of cracks require sensor use. Further, crack initiation often starts from the reinforcement or the aggregate component of reinforced concrete and so can be hidden from sight at conception. Corrosion is a critical consideration in Canada because of the use of de-icing salts during the winter months. These salts can penetrate the concrete cover and accelerate the corrosion process in the reinforcing members. The internal damages discussed are important safety considerations because they are not readily identifiable and can have detrimental impacts on the strength and long-term durability of the structure.

Research on the internal condition of RC structures has been predominantly carried out by contact methods. However, implementation of these contact-based approaches is hindered by 
the need for surface preparation and adhesion and the potential need for a large number of sensors, especially in the case of large RC structures such as bridges or dams.

Another difficulty in using NDT on reinforced concrete structures lies in the use of concrete as one of the main mediums. Concrete is a non-homogeneous material made up of cement and various aggregates. The use of concrete in large civil-structures that are critical to the safety of public and economic stability predicates that the status of the structure through analysis of the condition of the concrete be readily available.

Air-coupled and contact impact-echo methods can detect in-situ damage, but can be limited in the location and types of damage the methods can detect depending on the resolution, number of sensors used, and the post-processing techniques employed [17, 18, 39, 46, 47, 49, 50, 58, 62, 67]. Oh and Popovics (2014) carried out testing on concrete slabs in order to present an imaging technique called C-scan stack images that clearly define near-surface damages using a Fast Fourier Transform (FFT) [62]. Oh and Popovics were able to successfully identify nearsurface voids and delaminations of depths between 25 and $65 \mathrm{~mm}$ in various concrete specimens [62]. Groschup and Grosse (2015) used micro-electro-mechanical sensor (MEMS) microphones in an array in order to identify Lamb wave modes and provide improved signal resolution and reduced the noise in the signal [46].

The impact-echo methods used to date are inefficient in the case of large structures, as the impact is applied at the sensor location leading to the need for a high number of data points for good resolution of the state of the structure. Thus, for the purposes of this research a wavelet transform-based approach for movable air-coupled impact-echo testing using sensors placed away from the impact location is proposed. The impact-echo method was chosen to investigate damage sensitive features of data processing techniques because of its capacity to detect in-situ 
damage in structures. Further, the impact-echo method has promising real time or pseudo-real time monitoring applications.

Machine learning has become increasingly prevalent in many different fields. It has been shown to be capable of low-level decision-making and has recently been used in increasingly complex and diverse applications. Machine learning is an exceptional tool for analyzing vast amounts of data and deducing patterns within the data. SHM is a field that stands to benefit greatly from the development of machine learning tools. SHM has moved to data-dense systems, utilizing numerous sensor types in order to continuously monitor infrastructure such as bridges and dams. These monitoring systems have benefited from increased computing power and analysis software in recent decades.

An artificial neural network (ANN) will be used in order to continue the work on the efficacy of machine learning for NDT methods. This study proposes an ANN application that automatically determines intact and damaged locations from a small training sample of impactecho data from a reinforced concrete beam. Further, an autonomous post-processing method will be developed, removing the need for user input following the initial setup of the system.

The outline of this thesis is as follows. Chapter 1 introduces the topic and outlines the problem and objective of the research. Chapter 2 gives an overview of the field of structural health monitoring and the current areas of research. Chapter 3 and 4 report testing carried out using the impact-echo method in laboratory and field settings, respectively. Chapter 4 also discusses the semi-automation of the method to reduce testing time and improve measurement location accuracy. The use of an ANN with a squared-distance algorithm to classify impact-echo results as damaged or intact is discussed in Chapter 5. Finally, Chapter 6 concludes the thesis with a summary of results and findings and a discussion of future research. 


\subsection{Problem Definition}

NDT methods have progressed markedly over the past decades because of increased attention from researchers. However, locating internal damage in RC structures in an efficient and conclusive manner remains a challenge. No one method is capable of determining all common defects and in many cases the testing method is cumbersome when applied over the potentially massive RC structure. Therefore, this research seeks to improve the efficiency and precision of impact-echo testing and to study the feasibility of using semi-automated testing for the discovery of internal damages in RC. The use of an ANN in order to aid in the classification of intact and damaged regions of the structure will also be looked at.

\subsection{Objective Statement}

The objective of this research is to help improve the assessment process of large reinforced concrete civil structures. This research aims to aid in the development of methods of discovery of internal defects in large reinforced concrete civil structures and by extension provide improved decision making tools regarding the maintenance requirements and safety of the structure. This investigation attempts to accomplish these outcomes by:

1) Applying the impact-echo technique on a specimen with in situ damages. The resulting data from the experiments are used to further develop post-processing of data from the impact-echo method in general. The post-processing is found to improve damage detection while also allowing for more expedient testing via increased impact-to-sensor spacing over what is reasonable using the FFT on the same data set. 
2) Reducing the need for user interaction with the analysis of the impact-echo method in order to stream-line the implementation of the method, remove subjective decisions by the user, and aid in developing a robust SHM system that becomes increasingly capable as information from subsequent tests and test sites is gathered.

3) Validate the post-processing method and artificial neural network efficacy through fieldtesting of a parking garage. Mechanical control of the test setup is pursued in order to further automate the process and improve accuracy of the testing.

\section{LITERATURE REVIEW}

While infrastructure has continued to age, technology and understanding of the physical world has advanced. The development of computers has enabled means of measurement, calculation, and experiment that were not possible prior to their introduction. As well, material sciences have taken great strides in understanding how materials react under different conditions and what materials are best for different applications.

An area of SHM that continues to advance are the methods of discovery of internal damages in RC structures. Because of the scale and location of many RC structures, testing is difficult to carry out, time consuming, and is subject to varying levels of accuracy and areas of application depending on the tool used.

\subsection{Non-Destructive Testing}

Non-destructive testing (NDT) allows structures to be analyzed without damaging the structure. Several methods of NDT exist, including but not limited to: various scanning techniques, visual 
inspection, chain dragging, fiber-optic sensors, computer vision, acoustic emission sensors, ultrasonic testing, and the focus of this research, impact-echo testing.

Traditionally, internal conditions could only be determined by methods such as chain dragging, sounding, or coring. Over the last several decades, the range of methods for internal damage detection of concrete structures has expanded significantly.

\subsection{Visual Inspection}

Visual inspection is the current practice in many situations when the objective is determining the health of a structure. Visual inspection relies on highly trained individuals to carry out the inspections, both a benefit and drawback of the method.

Visual inspection is expensive and infrequent in many cases, with inspection occurring every year or every other year. While this schedule of inspection may be appropriate for maintenance purposes, safety and prevention of catastrophic failures would improve with increased inspection frequency.

The reliance on visually inspecting structures also leaves questions about the condition of the structure, especially with reinforced concrete. Cracks and evidence of corrosion of steel reinforcement in reinforced concrete can be detected with only a view of the external face of the structure. The extent of the damage, however, is difficult to judge. Further, damage such as debonding, delamination, and voids are generally undetectable through visual inspection. It is therefore clear that visual inspection can be improved upon as the standard monitoring technique. Sensor-based monitoring has the potential to fill the gaps in data collection and accident prevention left by reliance on visually inspecting structures. 


\subsection{Advantages of NDT}

One advantage of NDT is that the structural capacity of the structure is not compromised. Further, many methods of NDT can be carried out with higher frequency than the current practice of visual inspection of structures such as bridges. Approaches such as modal analysis, fiber-optics, acoustic emission sensing, and strain gauges can all provide timely information that serves as confirmation of structure performance in-between scheduled visual inspection.

If NDT methods such as impact-echo, ground-penetrating radar (GPR), and computervision can be automated, they could be carried out as often as would be practical or needed, increasing the amount of information about the structure and improving safety.

\subsection{Shortcomings of NDT}

Implementation of sensor-based approaches to SHM can be expensive. For example, large quantities of data are gathered using the impact-echo and pitch-catch techniques, requiring powerful computing to analyze, and substantial storage space. The cost of the system can be justified if the life of infrastructure can be improved as a result of implementation. The onus therefore lies, in part, with research such as this to create systems that are reliable and effective enough to warrant the cost of the technology.

Another problem with NDT is that no one method can gather all the information needed to do a fully comprehensive analysis of the structure in question. The need for multiple sensor types increases cost, and increases the complexity of the analysis. The lack of viable automated options in NDT is another reason the applications have not historically gained widespread use. The NDT field is rapidly expanding in general, however, and several promising papers have been published. For example, Schickert (2012) proposed an ultrasonic sensing system coupled with a SAFT system in order to create three-dimensional images of concrete structures [19], and Ham 
and Popovics (2015) proposed an automated contactless sensing system using ultrasonic sensors for concrete structures [49].

\subsection{State-of-the-Art}

Non-destructive testing of RC structures has made major advances in the last several decades, with sensor-based approaches becoming increasingly viable and more comprehensive than former methods such as chain dragging and visual inspection. All of the advances made reflect efforts to improve safety and reliability of infrastructure as well as attempt to limit the financial burden of aging infrastructure, as mentioned in the Chapter 1. Some of the areas of critical investigation in the field are fiber-optic sensors, acoustic emission sensors, pitch-catch techniques, including use of ultrasonic sensors, and impact echo techniques. Imaging techniques have also been improved, as researchers attempt to link data to interpretable results. Background information on many of the possible methods is subsequently provided, with particular attention paid to the methods leveraged for this research.

\subsubsection{Contact Applications}

NDT methods can be grouped into contact and non-contact types. Several contact methods of damage detection are subsequently discussed.

\subsubsection{Impact-Echo Method}

Impact-echo is a method of NDT involving an excitation source and a receiver. An instrument to provide excitation strikes the specimen and a receiver on the same side of the structure then measures the waves created in the structure. The collected signals are further processed to determine the state of the structure. The receiver can be contact or non-contact in nature and gather data in one of many different forms, including acceleration or sound pressure. The data is 
generally gathered in the time domain and post processed to retrieve the necessary information about state of the structure at that location. It is common to observe information in the frequency domain through a form of the Fourier Transformation after collection $[46,49,50]$. The frequency domain is of interest because the response in the frequency domain changes significantly depending on the internal conditions of the $\mathrm{RC}$ structure and is more easily understood and visible than the raw data response, whether acceleration, sound pressure, or another data type is used. The position of the excitation source, receiver, and damage identified have all been shown to have an effect on the data gathered by the receiver [62].

Impact hammers are one of the most common tools for impact-echo testing. The main feature of an impact hammer is the head that contains a moveable part that is used to impact the structure and a force sensor so that the amplitude of the impact force is known. The impact hammer is particularly useful because the force applied by it is tabulated over the course of an impact. Access to the force of impact is important because the amplitude of the resulting wave will depend on the magnitude of the force. If the force of impact is available, it is a simple matter of scaling the data using the magnitude of the force in order to more easily compare the postprocessed results at different locations.

As an alternative to impact hammers, excitation can be produced manually. A common means of manual excitation is a steel ball and rod configuration [39, 49, 67]. The drawback of manual excitation is that the force applied to the structure is not known. This can be overcome by normalizing the obtained data to one of the distinguishable features in the received signal; generally, the early arriving longitudinal waves are used for this purpose [41, 62].

Kee and Zhu (2009) used impact-echo testing to determine the depth of breathing cracks in concrete structures [55]. The findings by Kee and Zhu showed that far-field measurements 
should be used in order to measure breathing crack depth and the crack depths found were in agreement with the theoretical values calculated [55]. The work by Kee and Zhu also found that air-coupled sensors could be used to find the breathing cracks as an alternative to accelerometers [55]. Oh and Popovics (2014) used the impact-echo method in order to test for in-situ damage in a concrete slab [62]. It was shown that flexural vibration plays a large role in the dominant frequencies found in the signals [62]. They also found that small damages with respect to sensor spacing are poorly detected and that deep damages through the cross-section of the specimen have higher frequency responses than shallower damages [62]. Equation 1 gives a relationship between the velocity of the wave in the structure, the depth of the surface of reflection, and the frequency of the wave [46]:

$$
d=\frac{\beta C_{p}}{2 f}
$$

Equation 1

where $\beta$ is a coefficient, generally taken as 0.96 for concrete, $C_{p}$ is the velocity of the longitudinal wave (P-wave), $d$ is the depth of the reflective surface, and $f$ is frequency of the wave [46].

\subsubsection{Acoustic Emission}

There are many applications of acoustic emission sensors in FRP reinforced concrete structures. These applications include determining propagation of cracks in the concrete medium and debonding damage at the FRP/concrete interface and several more $[40,44,54,59,60,64,66$, 81].

Acoustic emission (AE) sensors pick up waves created when energy is released in a medium [37, 81]. Events that result in a damaged state of the structure release energy and therefore can be picked up by AE sensors. AE sensors output a voltage based on the input 
received and can be used in real-time monitoring applications [14]. The response output by the AE sensor can be used to determine if a damage event takes place and to determine the extent of the damage. The frequency of the AE sensor used is highly dependent on the material of interest, with an upper range of $60 \mathrm{kHz}$ for concrete, $150 \mathrm{kHz}$ for fiber reinforced polymer reinforcement, and $300 \mathrm{kHz}$ for steel [14].

The use of AE sensors can be holistic, monitoring the health of the structure overall, or more specifically, providing information on particular damage features such as cracks in concrete structures [14]. Further, the application of AE sensors can be used to monitor the crack phase of the concrete [14]. Concrete moves from tensile cracking to shear mode cracking as the structure moves towards failure [14]. Secondary features of cracking such as intensity and the level of health can be drawn from the AE signals [14].

AE sensors can be placed on reinforcement to measure damage events in the reinforcing members, which works particularly well in fiber reinforced polymer reinforcing bars because of the fibrous nature of the material that leads to multiple high-energy events before failure of the material $[12,81]$. AE sensors can also be placed on the exterior structures to detect damage [14].

AE sensors are generally implemented with the construction of a structure, but can also be added to pre-existing structures as long as the element being monitored can be accessed. AE sensors, like fiber-optic sensors, are only capable of observing changes in the condition of a structure. AE sensors cannot detect damage that precedes the implementation of the sensor because of the nature of the sensors; detecting waves from energy that is released from damage events $[14,37]$. This feature of AE sensors dictates that AE sensors be used for monitoring over time and not for maintenance checks because of the cumulative nature of the damage detection. 


\subsubsection{Fiber-Optics}

Fiber-optic sensors are another option for use in structural health monitoring. Fiber-optic sensors employ light as a means of conveying signals and, as a result, are highly efficient, suffering from very low attenuation of signal over the course of transmission compared to other methods [51]. Fiber-optic sensors are generally used to measure strain in reinforced concrete structures and work well for this application because the entire length of the cable can be used to detect strain [51]. These features are advantageous over conventional strain gauges that only read strain at the location of the head of the sensor.

The drawback of $\mathrm{AE}$ and fiber-optic sensors is that they must be connected to data collection systems by wires and numerous sensors are required to monitor large structures, thus increasing the costs incurred on sensors and the equipment needed to support them.

\subsubsection{Modal Analysis}

Modal analysis is another common method of SHM. This method often views the structure holistically and compares the mode shapes across time to detect damages. Some of the problems modal analysis applications have are their susceptibility to environmental conditions, difficulty in pinpointing damage location, and the need for large numbers of sensors in certain instances. This leads to cumbersome datasets, depending, of course, on the application and scale of the structure being observed [57].

Recently, cameras have been employed to carry out modal analysis [75]. For example, Yang et al. (2017) developed an output-only algorithm capable of pulling out mode shapes from the captured images [75]. The information acquired can be used to calculate the modal frequency and damping ratio of the structure. Further, the method opens the door for modal analysis at a 
much higher resolution than previously possible using a solely sensor based approach because each pixel can act as a virtual sensor [75].

\subsubsection{PUNDIT and Ultrasonic Pulse Velocity}

One of the earliest applications of the PUNDIT for NDT was the measurement of the time of flight of a known ultrasonic wave. The time of flight of a wave measured between two ultrasonic transducers coupled to concrete can be used to make approximations of the modulus of elasticity and compressive strength of the concrete [21]. This is desirable because it avoids damaging additional cylinders in the case of compressive strength testing, as well as Young's modulus testing, and the negates necessity of an apparatus to measure explicitly the strain in the cylinder under load.

Mohammed and Mahmood (2016) observed the effect of aggregate size and brick aggregate on the results from the PUNDIT [21]. It was found that aggregate size has a noticeable effect on the velocity of the wave in the concrete and therefore causes variation in the calculated compressive strength of the concrete tested [21]. Mirmiran and Wei (2001) used ultrasonic pulse velocity (UPV) to interrogate fiber reinforced polymer retrofitted concrete [26]. It was found that the UPV increased by $6 \%$ under high axial loads [26]. Further, the UPV damage index performed better than the axial strain energy under $50 \%$ of the ultimate strength of the concrete [26].

\subsubsection{Ground Penetrating Radar}

Ground penetrating radar (GPR) has been used extensively and is especially well suited for reinforcement detection in concrete structures. GPR uses ultrasonic waves and the time of flight of the waves to determine reflective surfaces within the concrete structure. The velocity along with flight time of the wave can be used to determine the depth of the reflective feature; 
generally reinforcement, flaws in the concrete, or the back wall of the structure. GPR is often used with an array of transducers that act in sending and receiving modes, giving GPR outputs high spatial resolution. This allows individual reinforcing members, void features, and concrete cover and slab depth to be identified [22-25].

The application of GPR for SHM is an ongoing area of research, as are many of the sensor-based applications in the field. Hugenshmidt and Roman (2006) carried out an extensive test on five bridges [22]. It was found that the concrete cover over the steel reinforcing bars was accurately determined in $77 \%$ of the test cases and the concrete thickness of the bridge was accurately found in 95\% of the test cases [22]. Saarenketo and Scullion (2000) give an overview of GPR application on roadways, showing the comprehensive test conditions in which GPR can be used and the breadth of infrastructure that can be tested by the method [23]. Varnavina et al. (2015) used a ground-coupled GPR antenna to investigate the condition of 11 bridges in Missouri, USA [24]. Varnavina et al. reviewed several parameters of GPR testing including the number of samples per scan, the range of the travel time, and traverse spacing, among others [24]. These parameters were compared by imaging the internal state of the bridge decks [24]. It was found that a range of $15 \mathrm{~ns}$ was reasonable and would necessitate a minimum of 256 samples per scan for adequate resolution of the data [24]. Traverse spacing dictates the resolution of the image and the detail of the imaging with respect to size and location of the damages, and so should be chosen with the level of resolution needed in mind. Cassidy et al. (2011) performed a comparison of ultrasonic pulse-echo and GPR on a 0.3 meter-thick reinforced concrete slab and determined both had reasonable performance in finding a target void in the specimen [25].

GPR is effective for detection of reinforcement in the structure and the thickness of the concrete. However, because only about 50 percent of the energy in the initial wave is present in 
the reflected wave from the air-concrete interface, delaminations and voids can be difficult to detect [67].

\subsubsection{Non-Contact Applications}

One of the pursuits of researchers in the area of SHM is that of a non-contact method of testing $[1-3,38,49]$. The benefits of non-contact testing include increased efficiency in testing as the procedure circumvents the need for adhering sensors to the surface of the structure and variation in surface conditions do not effect results to the same degree as contact methods $[1-3,38,49]$. Four non-contact methods are described in further detail in this section.

\subsubsection{Thermal Imaging}

Thermal imaging has become more common as technology improves and as the cost of the equipment becomes less prohibitive. Thermal imaging uses heat signatures in the form of infrared rays and allows visualization by humans by converting the infrared information to the RGB spectrum. The basis of the use of thermal imaging on concrete structures, and specifically bridges, is that the structures will heat up and cool down over the course of the day and may do so at different rates if the internal state of the structure is not uniform. Thermal imaging is highly sensitive, with cameras able to detect changes of temperature as low as $0.08{ }^{\circ} \mathrm{C}$ [30]. Clark et al. (2003) used an Agema Thermovision 900 Camera outfitted with a 20 by $10^{\circ}$ lens to image a concrete bridge as well as a masonry bridge [30]. The researchers concluded that delaminations could be found in the concrete bridge, with all but two of the known delaminated areas correctly identified [30]. The delaminations found were as small as $20 \mathrm{~cm}$ in diameter [30]. The nature of the masonry bridge made it more difficult to quantify the results, with two possible damages found that were attributed to possible moisture content in the fill between the masonry blocks [30]. 


\subsubsection{Computer Vision}

One of the most rapidly advancing areas in SHM involve the use of computer vision-based applications. Computer vision refers to the use of images collected via camera to determine areas of damage with further processing. The processing power of computers available today, along with improved image processing algorithms, has led to numerous investigations into the use of computer vision to detect damage in structures.

Computer vision has been used to look at damage by identifying surface damages that are visible to cameras as well as by applying algorithms to draw out damage-sensitive features that may be due to internal damage [75]. Damages such as cracks, corrosion, and loosened bolts are identifiable using computer vision [31-33]. Some of the issues that are encountered by computer vision-based approaches are different lighting from image to image, limited data available for training sets, and the need for damage-specific algorithms [34, 35].

\subsubsection{Non-contact ultrasonic testing}

Ultrasonic sensors can also be used for structural health monitoring. These sensors take many forms, as the governing feature is that they are able to detect waves with frequencies higher than $20 \mathrm{kHz}$, which is higher than audible frequencies for human hearing.

The nature of ultrasonic NDT requires a sender and receiver, both of which must be able to work in the ultrasonic frequency range. Some sensors are capable of acting as both the sender and receiver of the signal $[42,58]$. Ultrasonic testing is generally carried out using a pitch-catch configuration. The setup involves sending a wave from a transducer that is collected by a receiver. This can be done either in contact with the structure or without contact. Pitch-catch sensing can be done using the electrostatic ultrasonic transducers [49]. Electrostatic transducers send waves when excited by an input voltage [49]. The input voltage can be uniform, in which 
case the frequency of the waves sent by the transducer is also uniform. The input voltage can also vary in order to send a signal with variable frequency [49].

Ham and Popovics (2015a) used non-contact ultrasonic transducers to determine damage in rail ties and scatter in concrete structures [49]. The research showed that the amplitude of detected surface waves is lowered by the presence of damage and that the sensor setup used delivers highly replicable testing owing to the non-contact nature of the setup when compared to contact testing using instrumentation such as accelerometers [49]. The MEMS sensors showed a SNR of $34.4 \mathrm{~dB}$ compared to $61.2 \mathrm{~dB}$ for the accelerometers used [49]. The energy of the recovered signals was evaluated using Equation 2 in order to give a measure of the wave scatter in the concrete [49]:

$$
S_{E}=\frac{1}{t_{1}-t_{0}} \int_{t_{0}}^{t_{1}}\left|v_{i}(t)\right|^{2}
$$

where $S_{E}$ is the signal energy, $i$, is the signal path used in the calculation, and $v$ is the signal from the receiver [49].

Berriman et al. (2006) used ultrasonic chirp signals over a frequency range of $150 \mathrm{kHz}$ to $650 \mathrm{kHz}$ to find reinforcement in concrete [38]. They found that time-frequency analysis with wavelets, the Hough transform, the Wigner-Ville representation, or short-term Fourier transform made it possible to identify reinforcement from signals that had a very low SNR [38]. Testing was performed with a double-sided approach with the sender and receiver on opposite sides of the specimen [38]. The research was carried out on $10 \mathrm{~mm}$ thick concrete specimens with $10 \mathrm{~mm}$ steel reinforcement running parallel to the $300 \mathrm{~mm}$ by $300 \mathrm{~mm}$ plan surface [38].

The benefits of non-contact sensing with ultrasonic waves are that the surface of the structure does not have to be prepared and the waves are robust against rough surface conditions. Further, the sensors do not have to be attached to the structure or the structure does not have to 
be contacted for each test point. This makes testing more efficient and improves the results compared to contact sensors when determining the fiber content of the structure because of the removal of the need to provide physical coupling to the structure [49]. The drawbacks that are associated with non-contact testing are that the waves undergo attenuation at the interface of the concrete in air, which can affect the signal-to-noise ratio in the recovered signal [49]. The depth of penetration of the wave to recover information about the internal condition of the structure is in part a function of the wavelength of the wave used [49]. The findings by Ham and Popovics (2015a) did show, however, that a reasonable SNR is possible with non-contact ultrasonic testing [49].

\subsubsection{Laser Vibrometry}

Lasers are beginning to be used more frequently in SHM. They have the advantage of being very precise and accurate as well as working over long ranges [73]. They can be prohibitively costly, however. Lasers are used for purposes similar to those of accelerometers, except they are noncontact and are used to find vibrational velocity instead of acceleration of the structure [48]. One particular study of interest uses laser vibrometry coupled with acoustic excitation to determine the flexural vibration modes present in a concrete structure at intact and internal damage locations [48]. The acoustic signal was sent over a spectrum of frequencies and the scanning laser Doppler vibrometer (SLDV) took measurements in two-dimensions from the surface of the concrete structure [48]. The method was successful and found near surface damages in the structure at a high degree of certainty [48].

\subsection{Comparison of NDT Methods}

The NDT methods mentioned above provide numerous options, each with strengths and weaknesses. Systemically, sensor-based applications face many of the same obstacles. However, 
the positive attributes and shortcomings of the different applications vary from method to method and help to explain the need for varied approaches. Table 1 shows a comparison of the advantages and disadvantages of some of the most common NDT methods.

Table 1: Comparison of advantages and disadvantages of NDT methods

\begin{tabular}{|c|c|c|}
\hline Method & Advantages & Disadvantages \\
\hline $\begin{array}{l}\text { Visual } \\
\text { inspection }\end{array}$ & - No instrumentation required & $\begin{array}{l}\text { - Subjective } \\
\text { - Internal condition of the } \\
\text { structure is unknown }\end{array}$ \\
\hline Chain dragging & $\begin{array}{l}\text { - Allows insight into internal } \\
\text { condition of the structure } \\
\text { - Cost effective }\end{array}$ & $\begin{array}{l}\text { - Subjective } \\
\text { - Not as precise as impact-echo }\end{array}$ \\
\hline Impact-echo & $\begin{array}{l}\text { - Detects in-situ damage } \\
\text { - Can detect multiple damage types } \\
\text { and locations through the cross } \\
\text { section of the structure }\end{array}$ & $\begin{array}{l}\text { - Time consuming } \\
\text { - Post-processing is necessary }\end{array}$ \\
\hline $\begin{array}{l}\text { Acoustic } \\
\text { emission } \\
\text { sensors }\end{array}$ & $\begin{array}{l}\text { - Real-time applications [64] } \\
\text { - Accurate crack detection [64] }\end{array}$ & $\begin{array}{l}\text { - Expensive to implement and } \\
\text { maintain } \\
\text { - In-situ damage is not } \\
\text { detectable } \\
\text { - May need replacement during } \\
\text { lifespan of structure }\end{array}$ \\
\hline $\begin{array}{l}\text { Fiber-optic } \\
\text { sensors }\end{array}$ & $\begin{array}{l}\text { - Quasi-real-time applications [51] } \\
\text { - Accurate crack detection [51] }\end{array}$ & $\begin{array}{l}\text { - Expensive to implement and } \\
\text { maintain } \\
\text { - In-situ damage is not } \\
\text { detectable } \\
\text { - May need replacement during } \\
\text { lifespan of structure }\end{array}$ \\
\hline Modal methods & - Gives holistic view of structure & $\begin{array}{l}\text { - Difficult to determine exact } \\
\text { locations of damage in the } \\
\text { structure } \\
\text { - May require large numbers of } \\
\text { sensors } \\
\text { - Sensitive to environmental } \\
\text { conditions }\end{array}$ \\
\hline PUNDIT & $\begin{array}{l}\text { - Gives estimation of Young's } \\
\text { modulus and compressive strength } \\
\text { - Provides indication of overall } \\
\text { condition of concrete [26] } \\
\text { - Crack detection }\end{array}$ & $\begin{array}{l}\text { - Double-sided access required } \\
\text { - Gives time-of-flight and not } \\
\text { location of damages }\end{array}$ \\
\hline
\end{tabular}




\begin{tabular}{|c|c|c|}
\hline $\begin{array}{l}\text { Ground } \\
\text { penetrating } \\
\text { radar }\end{array}$ & $\begin{array}{l}\text { - Continuous image instead of point- } \\
\text { by-point inspection like impact-echo } \\
\text { [23] } \\
\text { - Detects multiple types of in-situ } \\
\text { damage [22] }\end{array}$ & $\begin{array}{l}\text { - Expensive } \\
\text { - Detection of deep damages } \\
\text { can be difficult [22] }\end{array}$ \\
\hline $\begin{array}{l}\text { Thermal } \\
\text { imaging }\end{array}$ & $\begin{array}{l}\text { - Small internal damages can be found } \\
\text { [30] } \\
\text { - Entire structure surface can be } \\
\text { observed in a single image } \\
\text { (depending on the size of structure } \\
\text { and resolution required) } \\
\text { - Non-contact }\end{array}$ & $\begin{array}{l}\text { - Different materials exhibit } \\
\text { different emissivity [30] } \\
\text { - Attenuation of radiation can } \\
\text { occur between the surface of } \\
\text { the structure and the camera } \\
\text { [30] }\end{array}$ \\
\hline $\begin{array}{l}\text { Computer } \\
\text { vision }\end{array}$ & $\begin{array}{l}\text { - Provide explicit images of externally } \\
\text { visible damage [4-5] } \\
\text { - Quasi-real-time applications for } \\
\text { complicated systems }[5,31,35] \\
\text { - Can be used for extensive areas and } \\
\text { at a distance from the structure }\end{array}$ & $\begin{array}{l}\text { - Internal in-situ damage is not } \\
\text { detectable } \\
\text { - Can be expensive to } \\
\text { implement and maintain }\end{array}$ \\
\hline $\begin{array}{l}\text { Contactless } \\
\text { ultrasonic } \\
\text { methods }\end{array}$ & $\begin{array}{l}\text { - Non-contact } \\
\text { - Works above general noise } \\
\text { frequency }\end{array}$ & $\begin{array}{l}\text { - Wavelength dictates depth } \\
\text { reasonable results [49] } \\
\text { - Attenuation of signal at air- } \\
\text { concrete interface }\end{array}$ \\
\hline $\begin{array}{l}\text { Laser } \\
\text { vibrometry }\end{array}$ & $\begin{array}{l}\text { - Non-contact } \\
\text { - Long-range sensor ability } \\
\text { - Highly accurate }\end{array}$ & - Costly instrumentation \\
\hline
\end{tabular}

\subsection{Scan Types}

Three common scan types are used to visualize internal damage when signal data is available.

These are the A-, B-, and C-scan. A-scans are plots of energy as a function of time [43]. The location in one dimension can be substituted for time if the location is known at a particular testing time, or the testing is carried out at discrete points. B-scans allow the location of damage to be seen through the cross-section [43]. GPR uses B-scans in order to determine the location of reinforcement or damage in the structure. C-scans show the plan view features of the structure [43]. These are useful to visualize where the damage is in structures with large surface areas, such as bridge decks. 
Further, the scan types can be combined in order to observe different features. For example, B-scan images can be combined and given transparent and opaque features and used to in plan view in a similar way to a C-scan. Imaging techniques such as SAFT and tomography are also ways to create planar images of internal damages that often have the same final form as A-, B-, or C-scans. SAFT and tomography are introduced in the subsequent sections.

\subsection{SAFT}

The SAFT method is capable of testing concrete structures with single-side access and the use of ultrasonic waves or acoustic signals from impact-echo testing. SAFT methods use signals and superimpose them to produce images of the element [15-18]. The method was originally implemented using the geometry of the setup and was later validated using inverse scattering theory $[19,20]$.

The SAFT method proposed by Schickert (2012) utilizes a transducer array to create a three dimensional image of the concrete structure [15]. The method was able to define back wall depths when damage did not impede the signal path and it was also able to determine small voids in the concrete specimen with a high level of detail [15]. One of the important considerations in the work by Schickert (2012) was the automation of the measurement system in order to reduce measurement time and produce consistent increments of sensor travel [15]. The system used a FLEXUS transducer array that has 48 transducers and was able to scan a $240 \mathrm{~mm} \times 720 \mathrm{~mm}$ area in 16 minutes using a $20 \times 10 \mathrm{~mm}$ grid and 2 minutes with a $20 \mathrm{~mm} \times 20 \mathrm{~mm}$ grid [15]. The time to carry out SAFT testing is critical because of the high density of signals that are collected, with up to 10000 measurements taken for every square meter of surface area [15]. Beniwal and Ganguli (2015a) extended the application of SAFT to account for the condition of the reinforcement interface at the concrete using the polarity of the rebar image [16]. 
Ganguli et al. (2012) demonstrated that SAFT can theoretically be used to determine horizontal crack location and depth in concrete structures [16]. Ganguli et al. expanded the use of waveforms from solely observing compression wave arrival to shear wave arrival information in order to focus the wave front information and suppress ghost images that can occur using SAFT [16]. The theoretical sensors used in the model were contact in nature but the formulation of the compression wave part of the method could be setup to include travel time of the wave in the air for non-contact testing. Shear waves do not travel through air and therefore only the compression-to-compression wave front can be manipulated in order to determine the crack location [16].

\subsection{Tomography}

Tomography is a method of determining damage from pertinent data and has been applied to impact-echo and ultrasonic testing data [27]. Tomography-based images are created with depth spectra information from the collected signals represented at a particular plane in the structure [27]. Liu and Yeh (2011) used impact-echo data in order to create depth images in concrete derived from frequency information in the data [27]. The method allows depth to be explicitly viewed as distance from the surface of the specimen as an alternative to viewing the damage location only in terms of frequency [27]. The method was used to successfully show internal cracks in the concrete specimens [27].

\subsection{Waves in Reinforced Concrete}

Waves are a large part of many areas of engineering because of the information that can be ascribed to them, the information that they can intrinsically carry, and the damage they can cause. Waves can exist in the solid specimen or interact at the interface of the specimen and air 
and leak into the air, making non-contact sensing possible [67], an attribute that was leveraged in this work.

Longitudinal, transverse, Rayleigh, and Lamb waves are four of the wave types present in excited structures. An important consideration for the use of waves for NDT is the material being tested. For RC structures, it is suggested that waves with a frequency of less than $100 \mathrm{kHz}$ be used in order to prevent excess scattering in the signal [36]. The scattering is prevented in concrete at lower frequencies because waves are most sensitive to damages greater than one-half the wavelength [29]. Wavelength decreases with increased frequency, evident from Equation 3, and depending on the nominal size of the aggregate in the concrete, the waves will become sensitive to the aggregate when the frequency is around $100 \mathrm{kHz}$.

$$
\lambda=\frac{C_{R}}{f}
$$

where $\lambda$ is the wavelength, $C_{R}$ is the velocity of the Rayleigh wave, and $f$ is the frequency of the wave. The subsequent sections provide background information on the waves noted above and how they pertain to the testing carried out in this research.

\subsubsection{Rayleigh Waves}

One of the most common waves used for NDT is the Rayleigh wave. This is due, in part, to the fact that only a single side of the structure is needed in order to send and receive Rayleigh waves [49]. The depth that Rayleigh waves can penetrate is also flexible because it is dependent on the wavelength of the wave, which is affected by the frequency of excitation [49, 52, 53]. Equation 3, found in Section 2.10, can be used to find the wavelength of the wave for a given frequency. Rayleigh waves are characterized by elliptical motion of the particle, shown in Figure 1. 


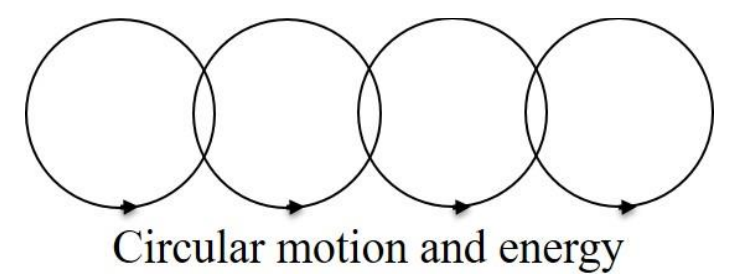

Figure 1: Rayleigh wave motion

The bulk of the energy in Rayleigh waves is contained in a depth below the surface of concrete equal to one wavelength [49].

\subsubsection{Longitudinal Waves (P-waves)}

Longitudinal waves (also known as compression or P-waves) are identified by particle motion parallel to the direction of the wave, visualized in Figure 2 [74].

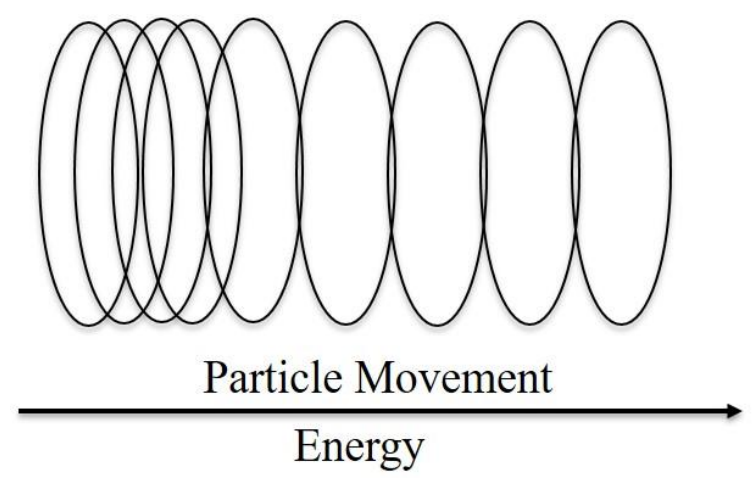

Figure 2: Longitudinal wave motion

Longitudinal waves, along with transverse waves, make up bulk waves [36]. Bulk waves exhibit large attenuation over long distances, making longitudinal and transverse waves poorly suited for damage detection over great distances, but appropriate for close-range applications [36]. The benefit of longitudinal waves over transverse waves is that longitudinal waves can 
transfer into liquid or gaseous mediums from solid mediums. This important feature allows noncontact readings to be taken.

\subsubsection{Transverse Waves (S-waves)}

Transverse waves (also known as shear or S-waves) are identified by particle motion orthogonal to the direction of the wave as displayed in Figure 3 [74].

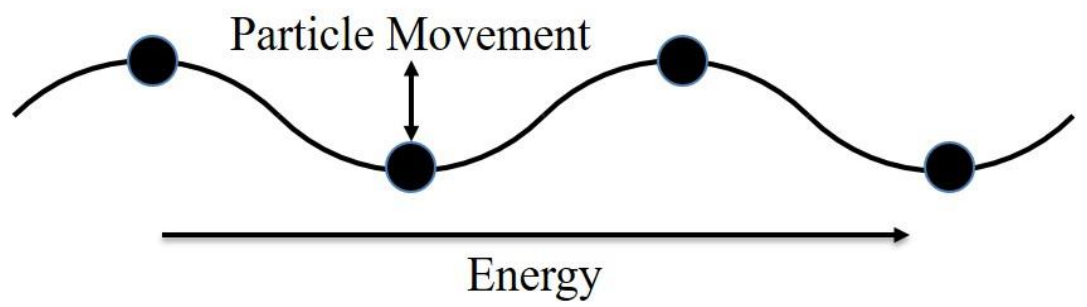

Figure 3: Transverse wave motion

Transverse waves are not used in non-contact applications because they do not propagate through air, mentioned above. Transverse waves are however suitable for applications using sensors such as accelerometers.

\subsubsection{Lamb Waves}

Lamb waves (pressure waves) that have an infinite number of modes are used in SHM applications [36]. Lamb waves exhibit different velocities with different frequencies and are, therefore, dispersive in nature [36]. In particular, the first symmetric $\left(\mathrm{S}_{0}\right)$ and asymmetric $\left(\mathrm{A}_{0}\right)$ modes of Lamb waves are used in damage detection because they experience less dispersion than higher modes [36]. The $\mathrm{A}_{0}$ mode is particularly useful in detecting damage in concrete structures because of the degree of displacement normal to the tested surface [29]. The $A_{0}$ mode is also known as the flexural mode [29]. 


\subsubsection{Snell's Law and the Second Critical Angle}

An important aspect of using waves as a means of damage detection in structures is the reflection of wave energy at interfaces. This interaction is governed by Snell's Law, which is given in one form by Equation 4:

$$
\frac{\sin \theta_{1}}{\sin \theta_{2}}=\frac{v_{1}}{v_{2}}=\frac{\lambda_{1}}{\lambda_{2}}
$$

Equation 4

From Snell's Law, several characteristics of the wave interaction at the interface of different mediums can be determined. For example, the critical angle and the second critical angle can be found. The second critical angle is of note because Rayleigh waves can be isolated at angles larger than this angle. At angles of incidence larger than the second critical angle, transverse waves (S-waves) and longitudinal waves (P-waves) do not refract forward in the concrete medium [49]. The second critical angle is given by Equation 5:

$$
\theta_{C 2}=\theta_{R}=\arcsin \left(\frac{v_{f}}{v_{R}}\right)
$$

Equation 5

where $v_{f}$ is the velocity of the wave in the fluid medium and $v_{R}$ is the Rayleigh wave velocity [49]. The Rayleigh waves that are produced by refraction moving from the fluid to concrete medium produce leaky longitudinal waves that return to the fluid at an angle of $\theta_{R}[49,68]$. It is important to note that the angle is taken from the vertical to the interface.

\subsubsection{Shadow Zone}

The shadow zone is an area where leaky surface waves are not produced [67]. The formula for the calculation of the shadow zone is given as:

$$
\mathrm{L}<\mathrm{h} \tan \theta
$$

Equation 6

where $\theta$ is the leaky angle of concrete and is assumed to be between 8 and 10 degrees [67]. 


\subsection{F1 Score}

The F1 score is a measure of the performance of a binary-type classification such as the result of processing the impact-echo data. The higher the F1 score, the better the performance of the method is for properly identifying the expected binary values. The F1 score is a function of precision and recall of the analysis. The F1 score can be calculated using Equations 7-9:

$$
\begin{aligned}
\text { precision }=\frac{\text { true positives }}{\text { true positives }+ \text { false positives }} & \text { Equation } 7 \\
\text { recall }=\frac{\text { true positives }}{\text { true positives }+ \text { false negatives }} & \text { Equation } 8 \\
F 1=\frac{2 \times \text { precision } \times \text { recall }}{\text { precision }+ \text { recall }} & \text { Equation } 9
\end{aligned}
$$

\subsection{Aikaike Information Criteria}

The Aikaike information criteria (AIC) can be used in order to simplify the interval used for the signals in both impact-echo and ultrasonic data analysis. This is especially useful for impactecho data where the useful information in the signal may be at different locations for different test point, especially if triggering is not used. A common window could be more easily found with the use of an impact hammer that triggers the start of the data collection. The AIC provides a global minimum that corresponds to P-wave arrival in the signal $[62,63]$. The AIC equation is given as:

$$
A I C(k)=k \times \log (\operatorname{var}(x[1, k]))+(N-k-1) \times \log (\operatorname{var}(x[k+1, N])) \quad \text { Equation } 10
$$

where $k$ is the index of the signal, $x$, and $N$ is the total number of samples in the signal [63].

The AIC value can be used in conjunction with wavelet analysis in order to determine the presence of the arrival of the P-wave [63]. In this case, the global minimum will be found to be 
at a similar location throughout analysis by several wavelet scales, corresponding to a likely Pwave arrival [63]. The AIC performs poorly when the signal-to-noise ratio (SNR) is low [63]. The utility of the AIC method for finding a consistent comparison point for signals taken without triggering is seen in Figure 4.

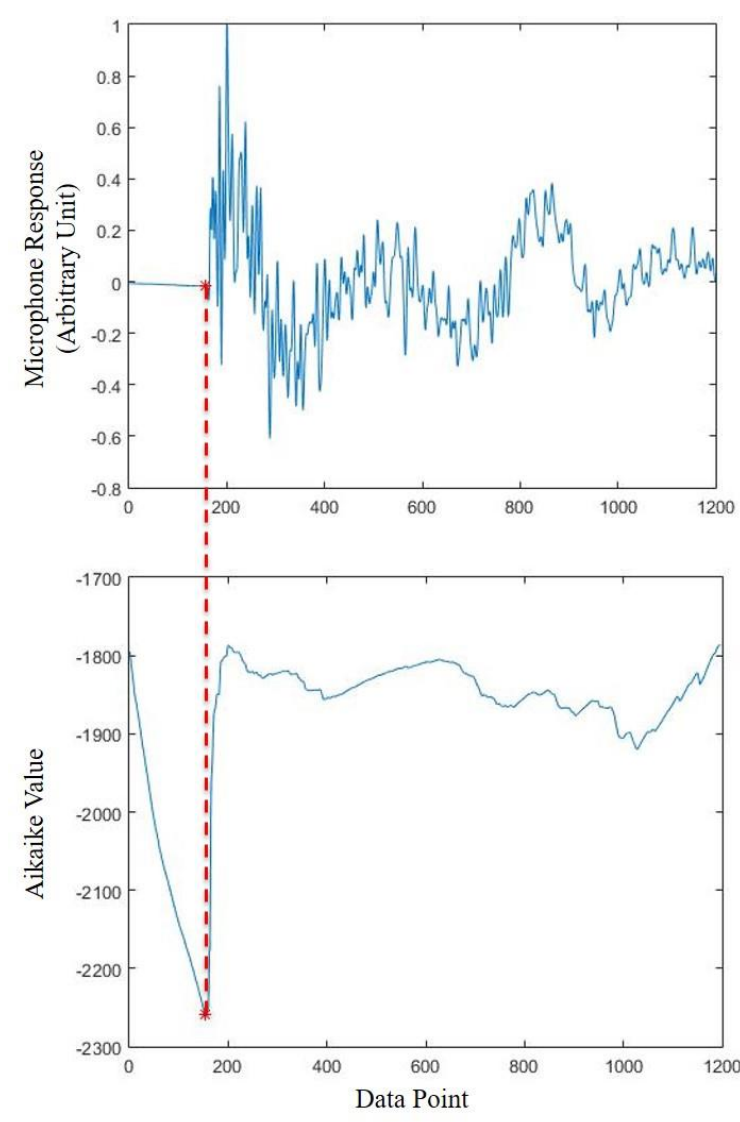

(a)

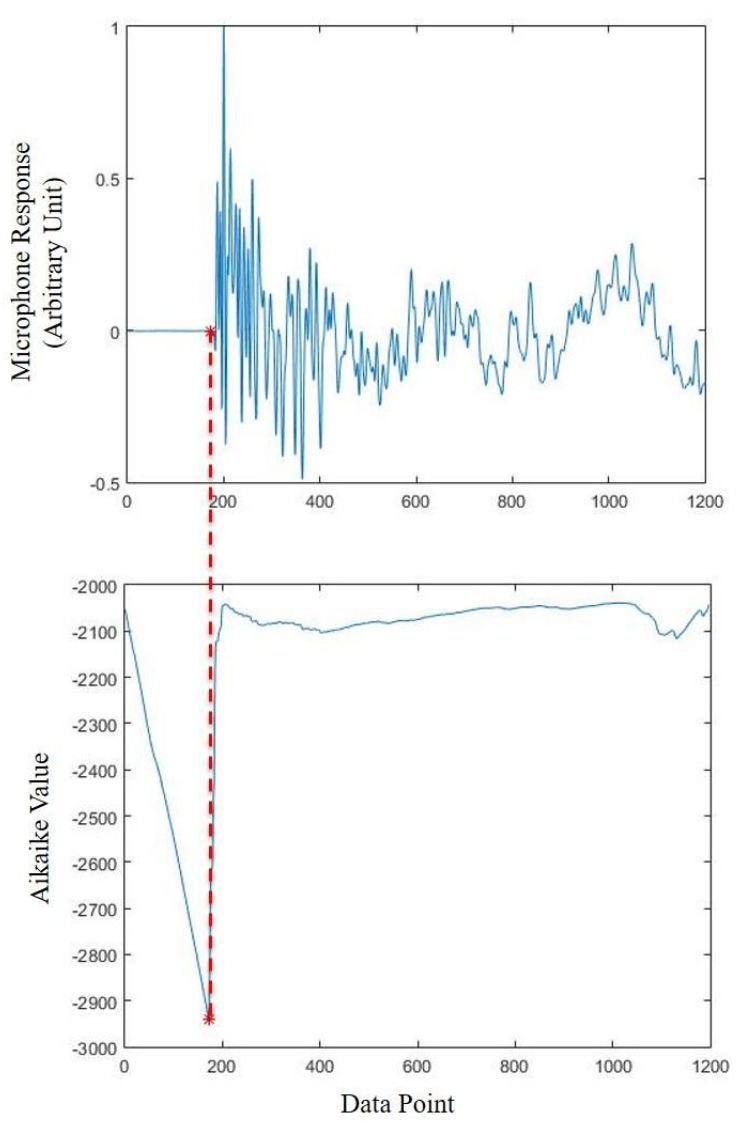

(b)

Figure 4: AIC from Impact-Echo Test 2 a) Point 4 (Intact) and b) Point 124 (Damaged)

From the figure the P-wave arrival is clearly identified as the minimum Aikaike value returned. This allows a consistent starting point of the signal to be used in analysis as long as there are is only one such feature in the signal. 


\subsection{Supervised Machine Learning}

Machine learning has become a larger part of SHM in recent years as the benefits of its implementation progress and knowledge of the algorithms becomes more widespread. Several types of machine learning exist. A non-exhaustive list of machine learning approaches includes previously mentioned ANNs, support vector machines (SVMs), genetic algorithms, deep learning, clustering methods, and Bayesian networks.

Machine learning can be used in order to make binary decisions about data outputs. For the case of impact-echo data, machine learning can be used in order to classify damaged and intact areas based on the collected data. The impact-echo method has been used with ANNs previously. Stavroulakis (1999) produced numerical examples of impact-echo testing with the application of an ANN [5]. The work focused on the effects of interlaying cracks in an arbitrary elastic material [5]. Xiang and Tso (2002) addressed delaminations and voids in concrete slabs in work [6]. A contact transducer was used to collect the impact signals and ANNs were used successfully to classify the damage types [6]. Even with high levels of noise, the ANN was able to correctly classify the intact, void, and delamination condition 92,88 , and 87 percent of the time, respectively [6]. Paulraj et al. (2013) used an artificial neural network (ANN) to determine damage sites on a steel plate [7]. The study employed statistical features such as Kurtosis, Root Mean Square, change in energy, and the number of slope changes in the signal [7]. The plate was excited using an impact and accelerometers that collected the signals for processing [7]. The features were then processed with an $\mathrm{ANN}$ and tested for the size of the training set relative to the number of test points available [7]. It was found that increasing the training set was beneficial to the performance in general, and that the largest training set tested $(80 \%$ of size of original data) gave the most accurate classification accuracy of $95 \%$ [7]. 
Non-destructive ultrasonic waves used for damage detection are another area ANNs have been used successfully as an alternative to impact-echo testing [8,9]. Legendre et al. (2001) successfully interrogated weld locations using ultrasonic waves [8]. A $1.5-\mathrm{MHz}$ contact transducer was used in order to excite the plate and the transducer in receiver mode captured the reflected waves [8]. Wavelet transform information was used as the features for the ANN training, resulting in correct identification of weld class in $90 \%$ of the signals [8].

\section{IMPACT-ECHO DAMAGE DETECTION}

In order to map internal damage in an RC beam, air coupled impact-echo testing was proposed. The signals obtained from the sensors were processed using wavelet transforms and relative energy in order to compare the results to those found from the traditional approach of using a FFT. The background and underlying theories of the impact-echo method are introduced in the following section.

\subsection{Impact-Echo Method}

Impact-echo is a method of NDT involving an excitation source and receiver(s). An instrument to induce waves in the structure strikes the specimen and a receiver measures the waves generated by the excitation. The data are generally gathered in the time domain and postprocessed to retrieve the necessary information about the health of the structure at the excited location. It is common to observe information in the frequency domain through a process of the FFT after collection $[46,49,50]$. The frequency domain is of interest because the response in the frequency domain changes significantly depending on the internal conditions of the RC structure, and is more easily understood and visible than the unprocessed signal retrieved by the receiver. 


\subsubsection{Microphones}

Various microphones are available as receiver options in the field of NDT. Microphones are used as a non-contact receiver option in either an air-coupled pitch-catch or impact-echo setup because of their ability to pick up waves that refract from concrete to air. There are several parameters of interest that may be different depending on the microphone. These parameters include sensitivity, frequency range, directional capabilities, and cost [49].

Two key factors in the selection of the type of microphone to be used are the environmental conditions the microphone will be used in and the data that is required. In applications of high ambient noise, directional microphones may be preferred, as they are designed to collect waves from only one direction. Devices are available commercially or can be built to increase microphone directionality [46].

Sensitivity is an attribute of great significance. Because of the attenuation of the signal as it passes from medium to medium, air-coupled damage detection in concrete structures often has signals of low amplitude. The higher the sensitivity, the less excitation needed to illicit a detectable response from the structure. High sensitivity is also useful in NDT because the magnitude of force of the impacts may be small. The two microphones used in this work are capable of detecting $20 \mathrm{kHz}$ frequencies with sensitivities of 34.4 and $41.7 \mathrm{mV} / \mathrm{Pa}$ at $250 \mathrm{~Hz}$, respectively, although the microphones are both PCB ICP Microphones.

\subsubsection{Wavelet Analysis}

Wavelets are used in signal processing for a multitude of reasons. Four of the most important are the retention of temporal signal information while spectral information is analyzed, de-noising capability, comparison across frequencies via scale, and the recovered coefficient of the applied wavelet. Using wavelet transforms for analysis is akin to using pass-band filters [57]. The filter 
can be shifted to a high- or low-pass filter depending on the scale of the wavelet used and the frequencies present in the signal.

This research employs the use of five different wavelets: Daubechies (DB) 4, DB 6, and DB 7 wavelet, the Mexican Hat wavelet, and the Morlet wavelet. The general form of the five wavelets is shown in Figure 5.

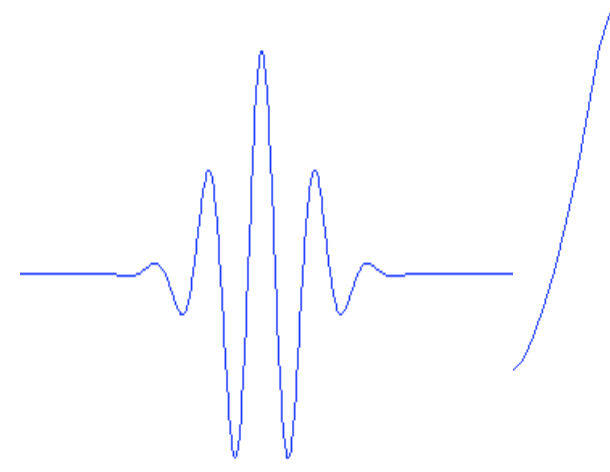

(a)

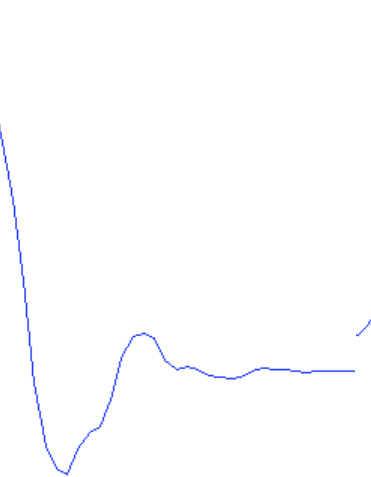

(b)

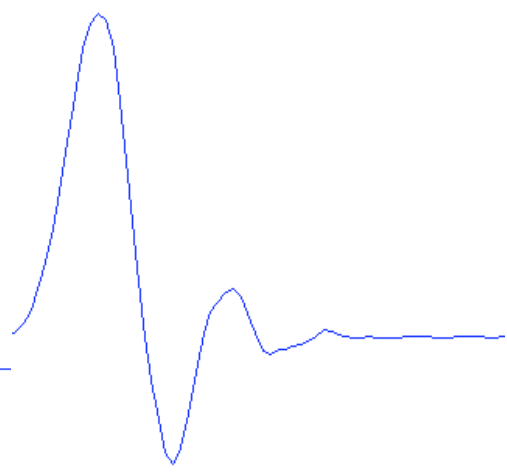

(c)

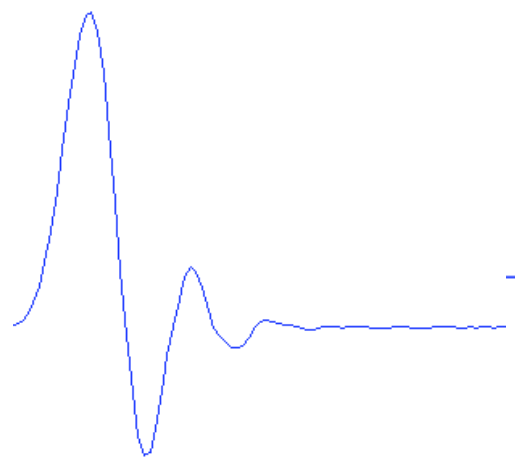

(d)

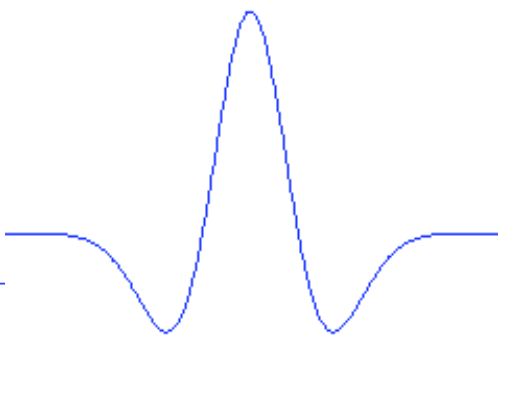

(e)

Figure 5: Wavelets used for wavelet analysis (a) Morlet Wavelet (b) DB 4 Wavelet (c) DB 6 Wavelet (d) DB 7 Wavelet (e) Mexican Hat Wavelet

The three DB wavelets are very similar in form. It can also be observed that the Morlet and Mexican Hat wavelet are similar in their symmetry but have different numbers of perturbations in their base form, causing them to behave differently in analysis. 
The main advantage of wavelet transforms over the FFT is that they give information about the frequency in relation to time, providing information about the temporal nature of spectral data instead of simple detection of the frequency in the signal. This feature of wavelet transforms was used in order to refine the scope of the imaging process and to investigate characteristics at specific instances in the signal.

Further, the parameters used for the wavelet dictate which features in the signal the wavelet will be sensitive to. Knowledge of the nature of the spectral data is therefore important, as the parameters set for the wavelet determine the effectiveness for the given application. When the frequencies embedded in a signal are unknown, the use of a FFT to determine the spectral makeup of a signal prior to the application of wavelet transforms is a beneficial coupling of the two processing methods. The way the wavelet is used, whether continuously or discretely, the type and condition of the signal, and the scale used all impact the effectiveness of the chosen wavelet.

Because the analysis was not time sensitive, the appropriate scale was more important than the particular wavelet that was used. For more time sensitive applications it may be prudent to thoroughly investigate wavelet types to optimize the application further.

Several factors must be accounted for when analyzing high-frequency signals. These factors include the Nyquist sampling theorem, the sampling rate of the recorded data, the frequency of interest, and signal length [65]. The Nyquist sampling theorem says that in order to properly identify a present frequency, the sampling rate of the given method of data recording must be at least twice the frequency of interest. In practice, it is prudent to have a sampling rate of 6-10 times the desired frequency if possible [65]. 
Errors at the end of the signal must also be taken into consideration [65]. It is possible to avoid them by analyzing a signal of length greater than that of interest and truncating the signal to the length needed for analysis. Quek et al. (2001) showed that a wavelet of scale p will have errors described in Table 2 when harmonic signals are analyzed [65].

Table 2: End of signal error using wavelets

\begin{tabular}{cc}
\hline Distance from end of signal (seconds) & Error (\%) \\
\hline $2^{-p}$ & 10 \\
$1.4^{*} 2^{-p}$ & 5 \\
$1.6^{*} 2^{-p}$ & 2 \\
\hline
\end{tabular}

Therefore, it is suggested that the minimum length of signal be $2^{1-p}$ seconds longer than the region of interest at the beginning and end of the signal [65].

\subsubsection{Continuous Wavelet Transforms}

The continuous wavelet transform (CWT) is a signal processing method available in damage detection. The name of the continuous wavelet transform is misleading because calculations must be carried out at discrete steps. The continuous nature of the method is derived from the fact that a wavelet coefficient is calculated at each step in a signal. As an example, a wavelet coefficient calculation is carried out using a mother wavelet given as $\left[\begin{array}{lll}1 & 0 & -1\end{array}\right]$ and a signal given

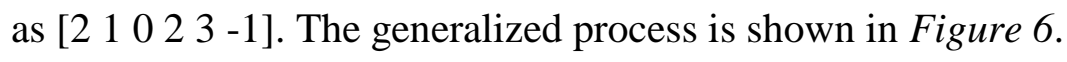




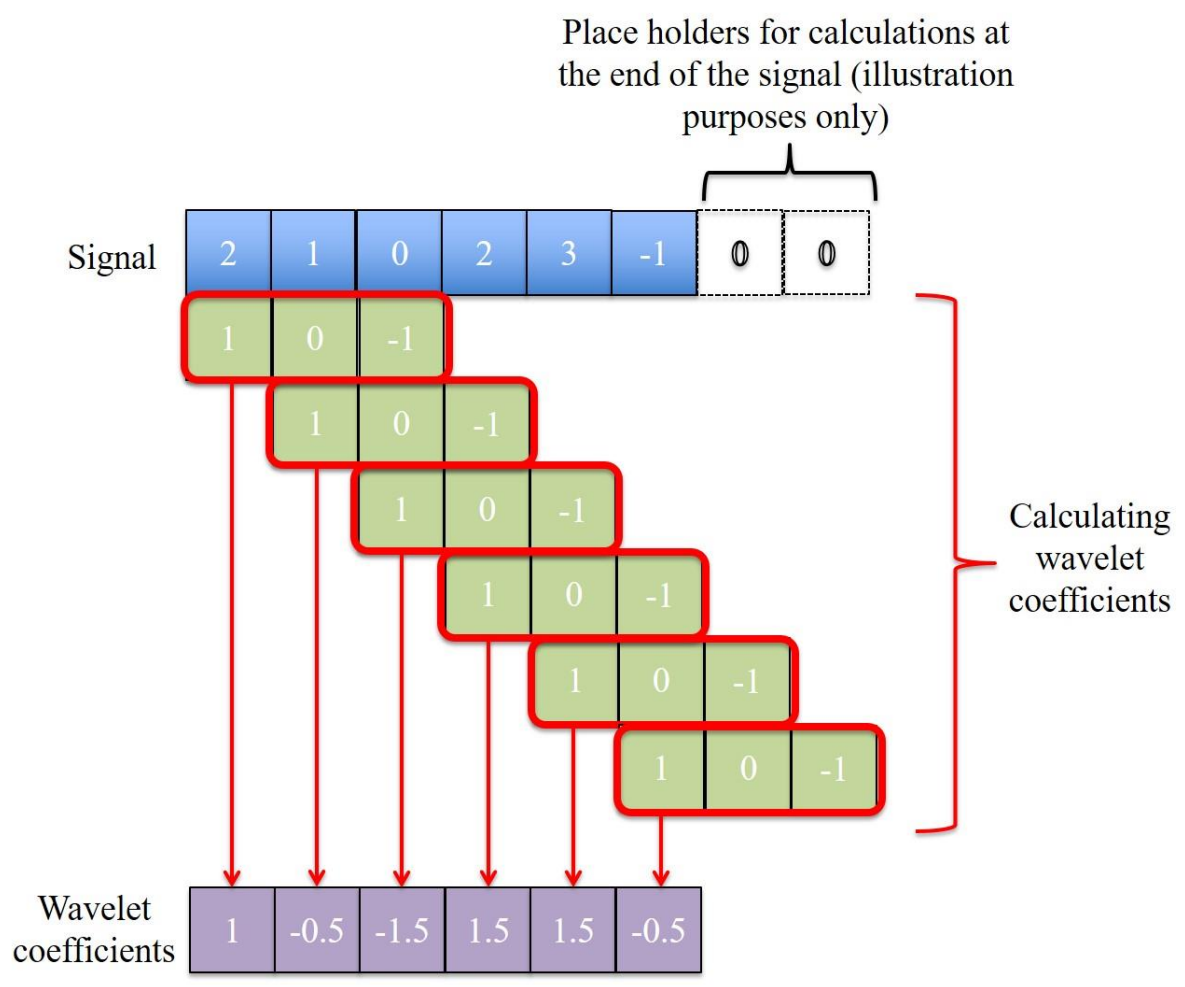

Figure 6: Implementing continuous wavelet transforms for arbitrary scale

The calculation of the coefficients shown in Figure 6 sums the areas under the curve of products from the wavelet and signal.

Two criteria must be satisfied when constructing mother wavelets for use in CWTs [57]. The integration of the mother wavelet should be zero and the square of the mother wavelet has a finite amount of energy [57]. Equations 11 and 12 represent these conditions, respectively [57]:

$$
\begin{gathered}
\int_{-\infty}^{\infty} \psi(t) d t=0 \\
\int_{-\infty}^{\infty}|\psi(t)|^{2} d t<\infty
\end{gathered}
$$

where $\psi(t)$ is the mother wavelet. 
One of the wavelets used in this study is the Mexican Hat wavelet. The Mexican Hat mother wavelet, $\psi(t)$, is shown as an example of mother wavelet construction in Equation 13:

$$
\psi(t)=\frac{2}{\sqrt{3} \pi^{\frac{1}{4}}}\left(1-t^{2}\right) e^{\frac{-t^{2}}{2}}
$$

CWTs return coefficients that correspond to the integral of the product of the wavelet and the signal to be analyzed, given in general form by $[45,57,61,65]$ :

$$
C_{a, b}=\int_{-\infty}^{\infty} s(t) \frac{1}{\sqrt{a}} \psi\left(\frac{t-b}{a}\right) d t
$$

where $s(t)$ is the function being analyzed and $\psi(t)$ is the mother wavelet. The variables $a, b$, and $t$ dictate the scale, shift, and time, respectively. The use of wavelet transforms allows similarities in the two functions (the wavelet and the signal being analyzed) to be determined, and is represented by the magnitude of the coefficient found from Equation 14.

\subsubsection{Discrete Wavelet Transforms}

Discrete wavelet transforms (DWTs) are also taken at specific time steps. Unlike CWTs, however, the transform is not performed at each discrete time step. DWTs use a scaling function that is not present in continuous wavelet applications in addition to the mother wavelet [57]. This scaling function acts as a low pass filter while the mother wavelet is akin to a high pass filter [57]. The conditions of wavelets for use in DWTs are also modified from those described for CWT wavelets. The mother wavelets must still satisfy the above criteria with respect to area and energy and must also have the integral of the scaling factor and the energy of the scaling factor equal to unity [57].

$$
\int_{-\infty}^{\infty} \phi(t) d t=1
$$




$$
\int_{-\infty}^{\infty}\left|\phi(t)^{2}\right| d t=1
$$

where $\phi(t)$ is the scaling function for the DWT [57]. The scaling function at level $j$ is found as:

$$
\phi(n)=\sum_{j=0}^{N-1} c_{j} \phi(2 n-j)
$$

Considering a signal of length $\mathrm{N}$ equal to 1000, the signal would first need to be increased to a length of $1024\left(2^{10}\right)$ with zero elements in order to carry out a DWT to the $10^{\text {th }}$ level. Application of the DWT gives an output at the lowest level of 512 coefficients (half the length of signal). Subsequent levels of DWT application further reduce the number of coefficients by a factor of two. Further, as the level of DWT application increases, the amount of signal information included in each coefficient increases as well. This phenomenon is explained by the Heisenberg uncertainty principle. The Heisenberg uncertainty principle relates time and frequency and shows the tradeoff between the two [57]. This can be seen in Equations 18 and 19 [57]:

$$
\begin{gathered}
\Delta t \Delta f \geq \frac{1}{4 \pi} \\
\frac{\Delta f}{f}=C
\end{gathered}
$$

where $C$ is a constant from the investigation of the proportionality of the change in frequency, $\Delta f$, and frequency, $f[57]$. Because of the relationships defined by the Heisenberg uncertainty principle, the time resolution increases as the frequency of observation decreases and the frequency resolution increases as the frequency of observation decreases [57]. An example of DWT calculation is shown in Figure 7. 


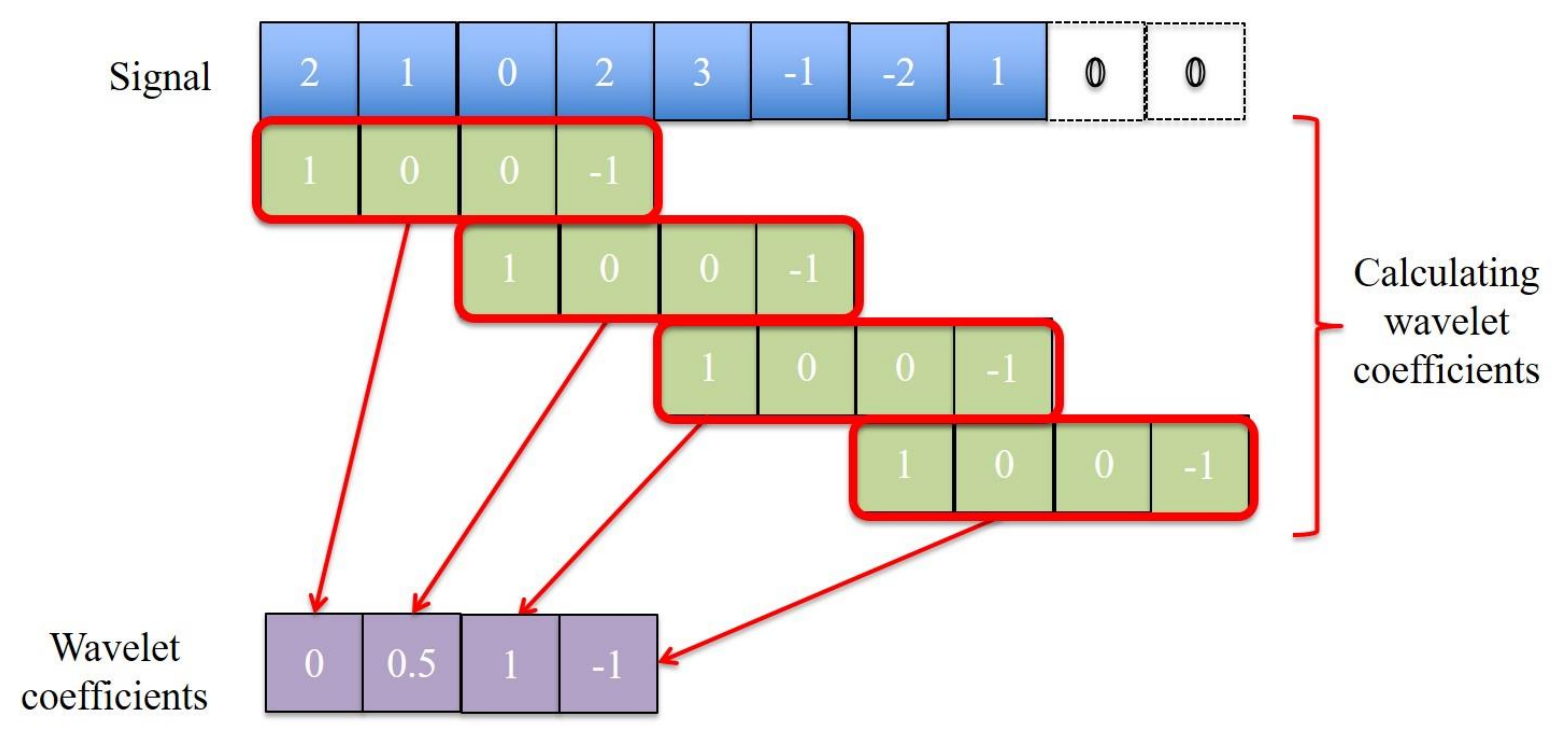

Figure 7: Implementing discrete wavelet transforms

The coefficients in Figure 7 are again found using the summation of area under the curve generated from the product of the mother wavelet and signal. The implementation of DWTs results in an approximate and detailed solution because of the implementation of the low and high pass filters and how they relate to the Heisenberg uncertainty principle. The first level of the discrete wavelet transform produces two sets of coefficients, each of length N/2. Subsequent levels of deconstruction occur on the coefficients from the low pass filter. A second and third level deconstruction applied to the signal in Figure 7 would produce results of length N/4 and N/8, respectively. Equations 20 and 21 give the detailed and approximate solutions of the application of DWTs:

$$
\begin{gathered}
D_{j}=\sum_{k \in Z} a_{j, k} \psi_{j, k}(t) \\
A_{J}=\sum_{j>J} D_{J}
\end{gathered}
$$


where $j$ and $J$ are the particular levels of the approximate wavelet deconstruction for Equations 20 and 21, respectively [57]. The signal is therefore represented by a combination of the approximate and detailed solutions and can be reconstructed using Equation 22 [56, 57]:

$$
f(t)=A_{J}+\sum_{j \leq J} D_{j}
$$

Equation 22

\subsubsection{Wavelet Scaling}

The necessity of scaling wavelets is a deterrent when considering the use of wavelets as a tool in damage detection. The scale used when calculating the wavelet coefficients changes the frequencies the wavelet is sensitive to. The smaller the scale used, the smaller the breadth of the signal analyzed. This leads to higher frequencies being identified and increased sensitivity to temporal changes in the signal. When the scale used is large, the wavelet will be wider relative to the signal being analyzed and less sensitive to high frequency data within the signal. The method presented in this study allows scaling to be simplified. Multiple scales are viewed together to draw out information from the signal, removing the need to test each wavelet scale individually.

\subsection{Visualization Techniques}

Several different methods of using spectral data in order to visualize the health of structures have been proposed previously. Oh and Popovics [62], Kundu et al. [58], Zhu [67], Hoegh and Khazanovich [13], Beniwal and Ganguli [18], Beniwal and Ganguli [76], and Zhang et al. [77], have all postulated different spectral-based visualization methods for damage detection in concrete structures. The methods available vary in complexity, and are at least partially dependent on the damage of interest. Oh and Popovics (2014) proposed a variation of the C-scan method, a horizontal plane in the spectral data that focuses on certain values that are taken to represent damage sensitive features [62]. The C-scan involves an excitation source and 
receiver(s) that are moved around the specimen in the same array [62]. Hoegh and Khazanovich used SAFT to study the condition of the concrete-reinforcement interface [13]. The A-scan carried out by Beniwal and Ganguli (2015b) used one stationary transducer and a roaming receiver that was placed at each of the pre-defined grid points [76]. This was done to create an array of data. Once the transducer was applied at all of the points, a post-processing method was applied to produce a B-scan of the scattering of field focus [76]. Zhang et al. (2012) carried out impact-echo testing in order to recover spectral data about delaminated zones and the bottom surface of the specimen in order to create spectral ratios that improve boundary definition of the delamination [77].

Kundu et al. (1999) describes a C-scan as a single transducer-receiver setup where one sensor acts as both the sender and receiver [58]. The Lamb wave scanning technique (L-scan) described in the work by Kundu et al. is similar to the C-scan method outlined by Oh and Popovics (2014) in that it uses an independent transducer and receiver with a space between the two $[58,62]$. The methods differ, however, in the type of waves that are analyzed and the types of damage that are present.

In the subsequent work, damage is visualized by a C-scan using spectral analysis of signals received from an impact-echo setup with microphones $100 \mathrm{~mm}$ and $200 \mathrm{~mm}$ from the impact and $5 \mathrm{~mm}$ from the surface of the reinforced concrete structure. The second test employs both microphones at $200 \mathrm{~mm}$ from the impact. The C-scans obtained from the relative energy of coefficients extracted from the application of scaled wavelet transforms are compared to the more conventional FFT C-scan. The two methods are subsequently compared for suitability of the given sensor setup. 


\subsection{Impact-Echo Experimental Setup}

The experimental setup for this work consisted of a $4000 \times 360 \times 300 \mathrm{~mm}$ simply supported reinforced concrete beam with in-situ damages as shown in Figure 9 (a). The strength of concrete at 28 days was $40.4 \mathrm{MPa}$. The damages in the specimen included voids, delaminations, and cracks in the concrete. Figure 8 depicts the construction of the specimen. Figure 8 (a) shows the reinforcement setup with $15 \mathrm{M}$ steel reinforcement on the left, $10 \mathrm{M}$ carbon fiber reinforced polymer in the middle, and $15 \mathrm{M}$ glass fiber reinforced polymer on right. The different reinforcement were used to be able to test for the detection of different reinforcement types in future work. Figure 8 (b) shows the general location of the damage in the plan view of the structure. The smaller damages were ultimately staggered for easier individual detection. The $100 \times 100 \mathrm{~mm}$ void and $300 \times 140 \mathrm{~mm}$ delamination were also switched to accommodate detection and the location of the lifting hooks placed in the specimen.

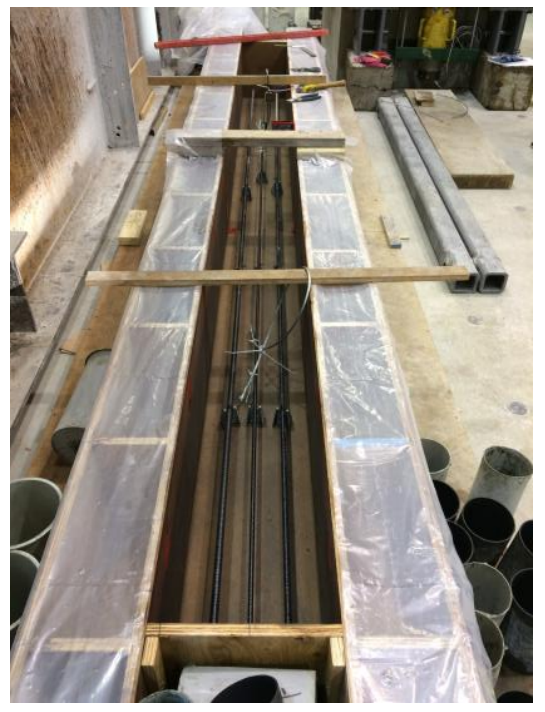

(a)

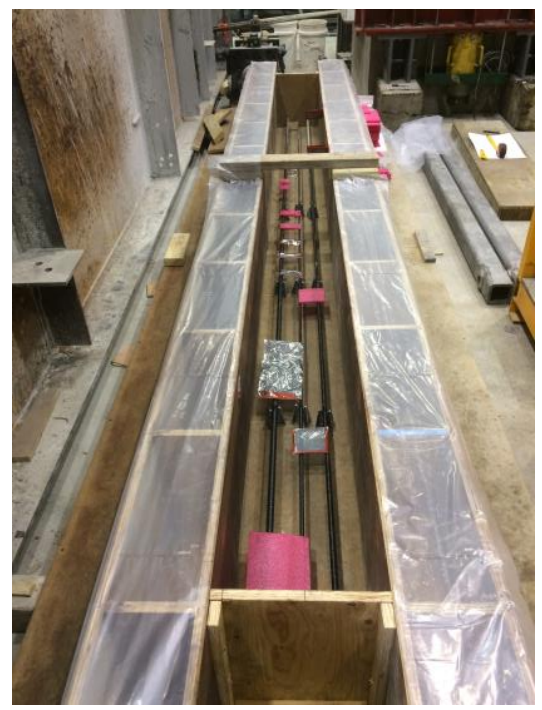

(b)

Figure 8: Experimental specimen construction 
Figure 9 shows the simply supported lab specimen and all damage scenarios generated for experimental studies; details of the damage scenarios are presented in the Table 1 . The damages vary greatly in size and were dispersed vertically through the cross section of the structure. This was done so the performance of the wavelet transformations could be compared to the performance of FFT for several applications that have been both successfully and unsuccessfully carried out in past work [62].

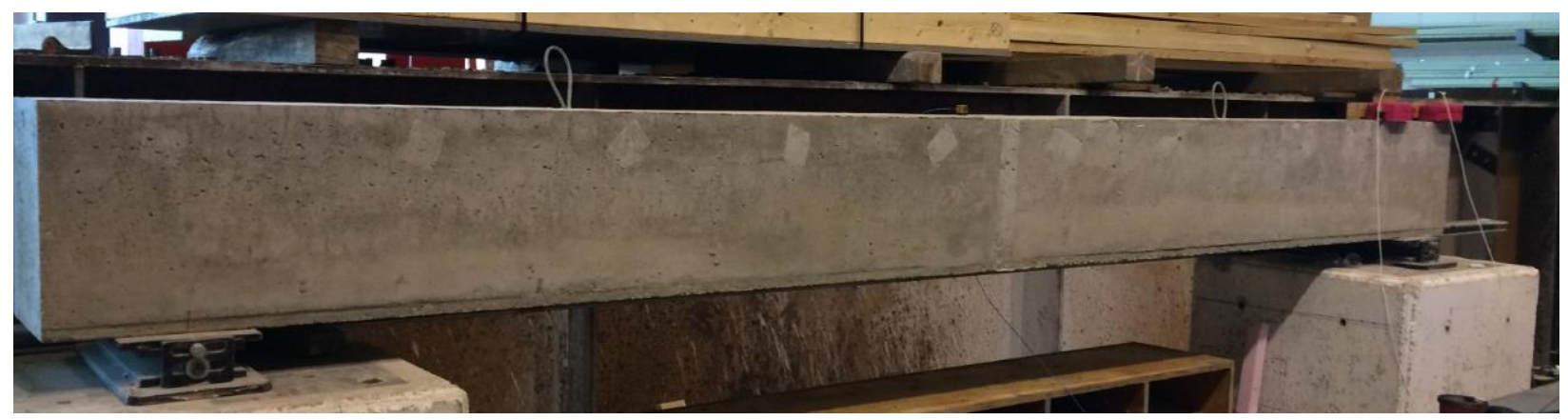

(a)
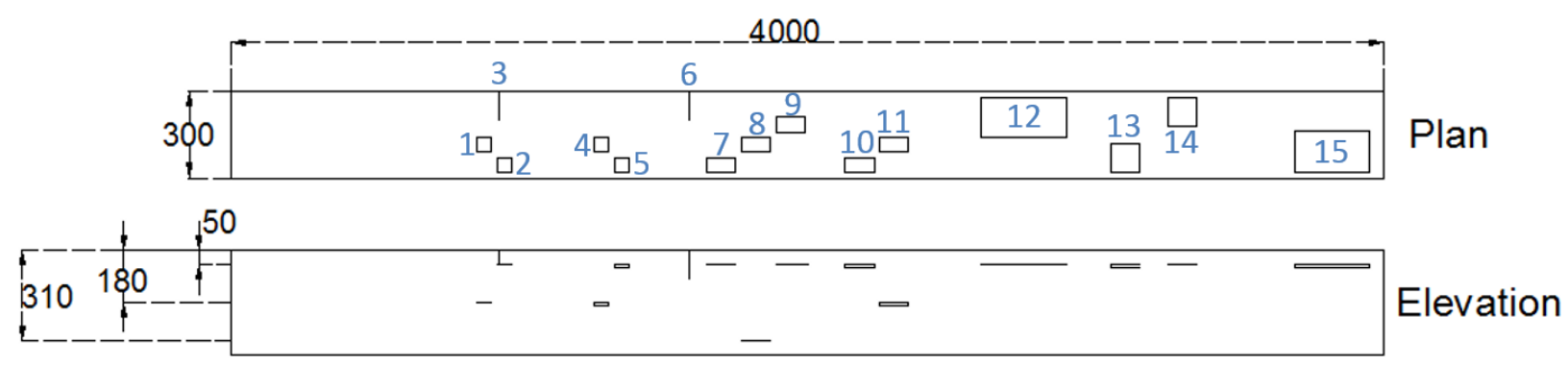

(b)

Figure 9: (a) Simply supported $4000 \times 360 \times 300 \mathrm{~mm}$ specimen and (b) Plan view (top) and elevation view (bottom) of the damages and damage locations in the reinforced concrete beam

Table 3: Damage types and dimensions

\begin{tabular}{llll}
\hline Damage scenario & Type of damage & Dimension $(\mathbf{m m})$ & Depth $(\mathbf{m m})$ \\
\hline 1 & Delamination & $50 \times 50$ & 180 \\
2 & Delamination & $50 \times 50$ & 50 \\
3 & Crack & $100 \times 50 \times(<1)$ & \\
4 & Void & $50 \times 50 \times 10$ & 180 \\
5 & Void & $50 \times 50 \times 10$ & 50
\end{tabular}




\begin{tabular}{llll}
6 & Crack & $100 \times 100 \times(<1)$ & \\
7 & Delamination & $100 \times 50$ & 50 \\
8 & Delamination & $100 \times 50$ & 310 \\
9 & Delamination & $100 \times 50$ & 50 \\
10 & Void & $100 \times 50 \times 10$ & 50 \\
11 & Void & $100 \times 50 \times 10$ & 180 \\
12 & Delamination & $300 \times 140$ & 50 \\
13 & Void & $100 \times 100 \times 10$ & 50 \\
14 & Delamination & $100 \times 100$ & 50 \\
15 & Void & $260 \times 140 \times 10$ & 50 \\
\hline
\end{tabular}

The longitudinal (P-waves), transverse (S-waves), and Rayleigh wave velocities were calculated based on the material properties of the concrete in the specimen. The transverse and Rayleigh wave velocities were calculated using Young's Modulus and Poisson's Ratio of the concrete, which were found to be $16559 \mathrm{MPa}$ and 0.12 , respectively. The Young's Modulus and Poisson's Ratio were found following the American Society for Testing and Materials (ASTM) C 469 standard [78]. The longitudinal wave velocity was found following the ASTM C 597 standard [79]. The equations for transverse and Rayleigh wave velocity in solids are given below [74]:

$$
\begin{gathered}
C_{s}=\sqrt{\frac{E}{2 \rho(1+v)}} \\
C_{R}=\frac{(0.87+1.12 v)}{1+v} \sqrt{\frac{E}{2 \rho(1+v)}}
\end{gathered}
$$

where $C_{s}$ is transverse wave velocity, and $C_{R}$ is Rayleigh wave velocity. The $v$ is the Poisson's Ratio of the concrete, $E$ is Young's modulus of elasticity, and $\rho$ is the density of the concrete. The longitudinal, shear, and Rayleigh wave velocities were found to be $4927 \mathrm{~m} / \mathrm{s}, 1785 \mathrm{~m} / \mathrm{s}$, and $2028 \mathrm{~m} / \mathrm{s}$, respectively. 
A simple rod with a ball bearing attached to the end was used as the excitation source and is a common means of the manual excitation of reinforced concrete structures $[39,49,67]$. The drawback of manual excitation is that the force applied to the structure is not known. This can be overcome by normalizing the obtained data to one of the distinguishable features in the received signal; generally the largest value in the signal or the early arriving wave (either longitudinal or shear waves) is used for this purpose [49, 62]. For this work the normalization was carried out using the largest value within each received signal. Two microphones were used to record the response of the specimen to excitation.

Two tests were carried out in order to compare the performance of FFT and wavelet transforms. Test 1 had the first microphone (MIC 1) positioned $100 \mathrm{~mm}$ from the excitation source and the second microphone (MIC 2) positioned $200 \mathrm{~mm}$ from the excitation source. The ambient noise in the laboratory where the specimen was located was high and so foam was used in an attempt to insulate the microphones as shown in Figure 2. Test 2 had both MIC 1 and MIC 2 positioned $200 \mathrm{~mm}$ from the excitation point. The microphones have sensitivities of 34.4 and $41.7 \mathrm{mV} / \mathrm{Pa}$, respectively. The microphone setup is shown in Figure 10.

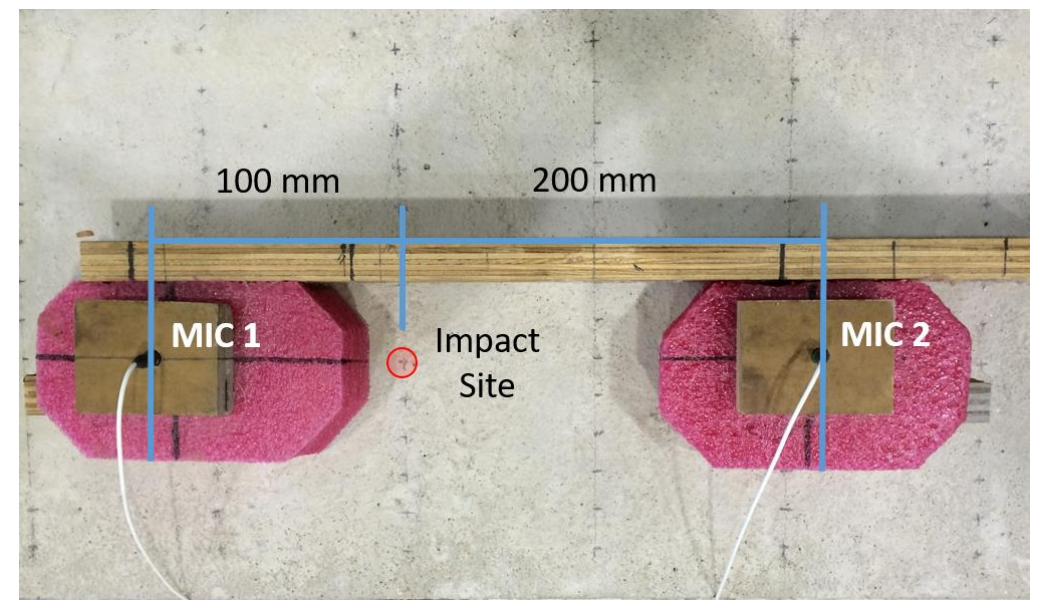

Figure 10: Example of microphone setup and impact location 
A total of 160 points were tested with the first test setup in order to determine the internal damage locations of the beam. Test points were taken at $300 \mathrm{~mm}$ increments over the longitudinal direction $(4000 \mathrm{~mm})$ of the beam and at $20 \mathrm{~mm}$ increments over the transverse direction $(300 \mathrm{~mm})$ of the specimen. The spacing was chosen in order to maintain the resolution of damage while illustrating damages can be found over distance, thus reducing the number of test points needed. The first row of data points was taken at $150 \mathrm{~mm}$ and the final row was taken at $3750 \mathrm{~mm}$. The second test setup allows for 126 and 140 points to be tested for MIC 1 and 2, respectively. The first row of data points was taken at $350 \mathrm{~mm}$ and the final row was taken at $3750 \mathrm{~mm}$. The same methodology was used as in Test 1, with the exception that the total sensor spacing was increased to $400 \mathrm{~mm}$.

The location of the test points meant that the first and last $50 \mathrm{~mm}$ of the specimen for Test 1 and the first $150 \mathrm{~mm}$ and last $50 \mathrm{~mm}$ for Test 2 were not directly measured, although reflected waves from those regions were contained in the measured signal. The sensor apparatus was obstructed from its testing position near the lift hooks in the specimen, and so several points were untestable. In Test 1 these points include the $80-180 \mathrm{~mm}$ points of the rows located at 750 and $1050 \mathrm{~mm}$ as well as $100-180 \mathrm{~mm}$ points in the rows at 2850 and $3150 \mathrm{~mm}$, seen in Figure 11(a). The range of test points $80-180 \mathrm{~mm}$ for the row at $750 \mathrm{~mm}, 100-180$ for the row at 1150 mm, and 180-200 for the row at $3150 \mathrm{~mm}$ were inaccessible for Test 2, seen in Figure 11(b).

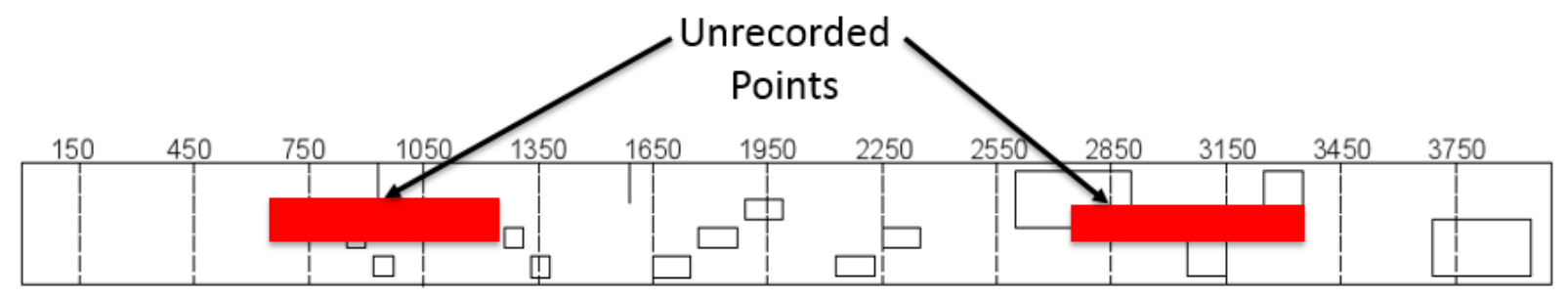

(a) 


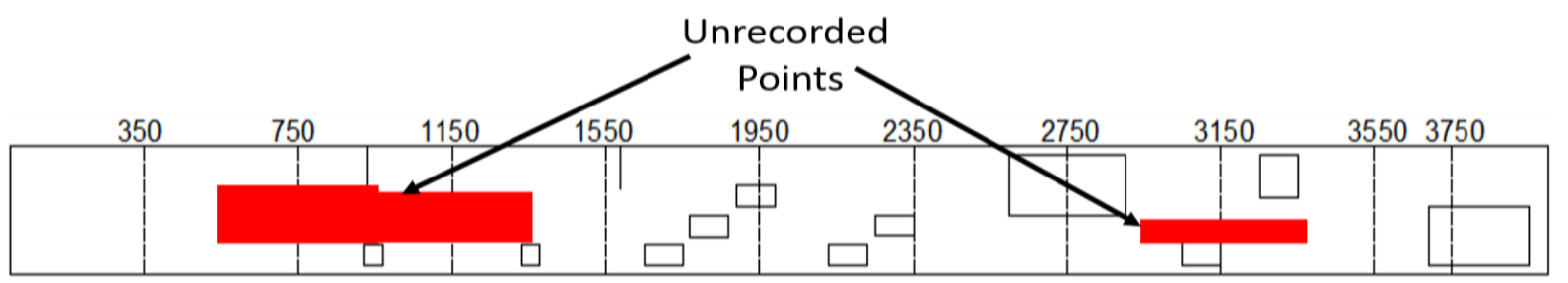

(b)

Figure 11: Regions of testing not accessible to sensors for (a) Test 1 and (b) Test 2

Figure 11 shows the location of damages with reference to the impact sites. The beam was excited along the dashed lines at increments of $20 \mathrm{~mm}$ with the microphone apparatus perpendicular to the dashed lines.

The DAQ used acquired signals from the $20 \mathrm{kHz}$ microphones with a sampling rate of 200 $\mathrm{kHz}$. The recorded signals were gathered for a five-second duration in LabVIEW. The signals were then truncated to 6 milliseconds, or 1200 data points, before data analysis was carried out. The truncated signal included a short period before the maximum positive value in the signal and is assumed to include the early arriving longitudinal wave.

\subsection{Impact-Echo Testing Results and Discussion}

Using the measured signals, FFT analysis and wavelet transforms were carried out in order to compare the performance of the damage detection with various types of damage scenarios presented previously in Figure 9(b) and Table 3.

\subsubsection{FFT Analysis}

Analyzing the frequency of signals in response to excitation of concrete structures is a proven method of damage detection $[46,49,50,62]$. The FFT was used in order to decompose the signals into their frequency components. For an initial step to map overall beam damage distribution, the desired locations were tested, and the measured data was processed using a FFT 
to find a range of damage sensitive frequencies by comparing points expected to have intact concrete characteristics and damaged concrete characteristics. Point 4 and 157 represent intact and damaged sites respectively, and are shown in Figure 12.

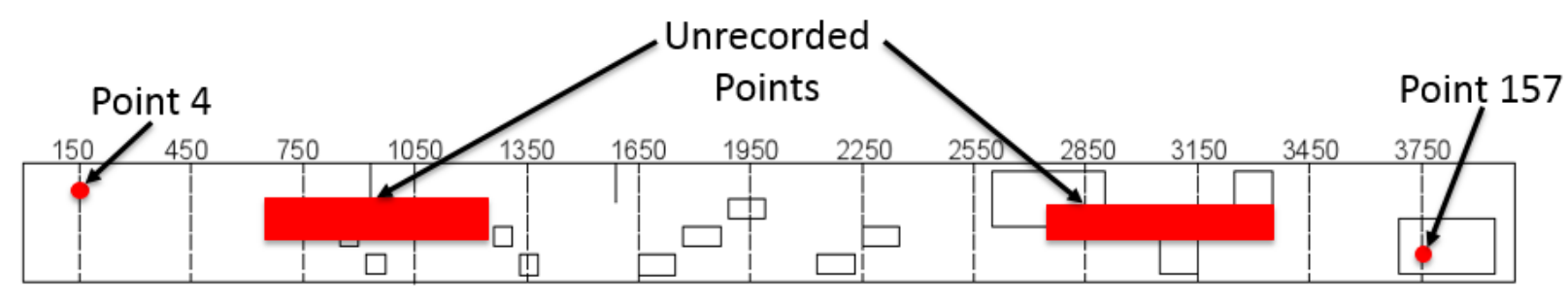

Figure 12: Test points 4 and 157 for Test 1

Figure 13 and Figure 14 shows the frequency responses of both microphones at Point 4 and Point 157 in Test 1 . From the results of the FFT analysis, it was concluded that frequency responses in the range of 4 to $7 \mathrm{kHz}$ are sensitive to damage, as the magnitude of the frequency responses in that range was far greater than at the intact point. This response was used as the criterion for damage detection throughout the collected signals.

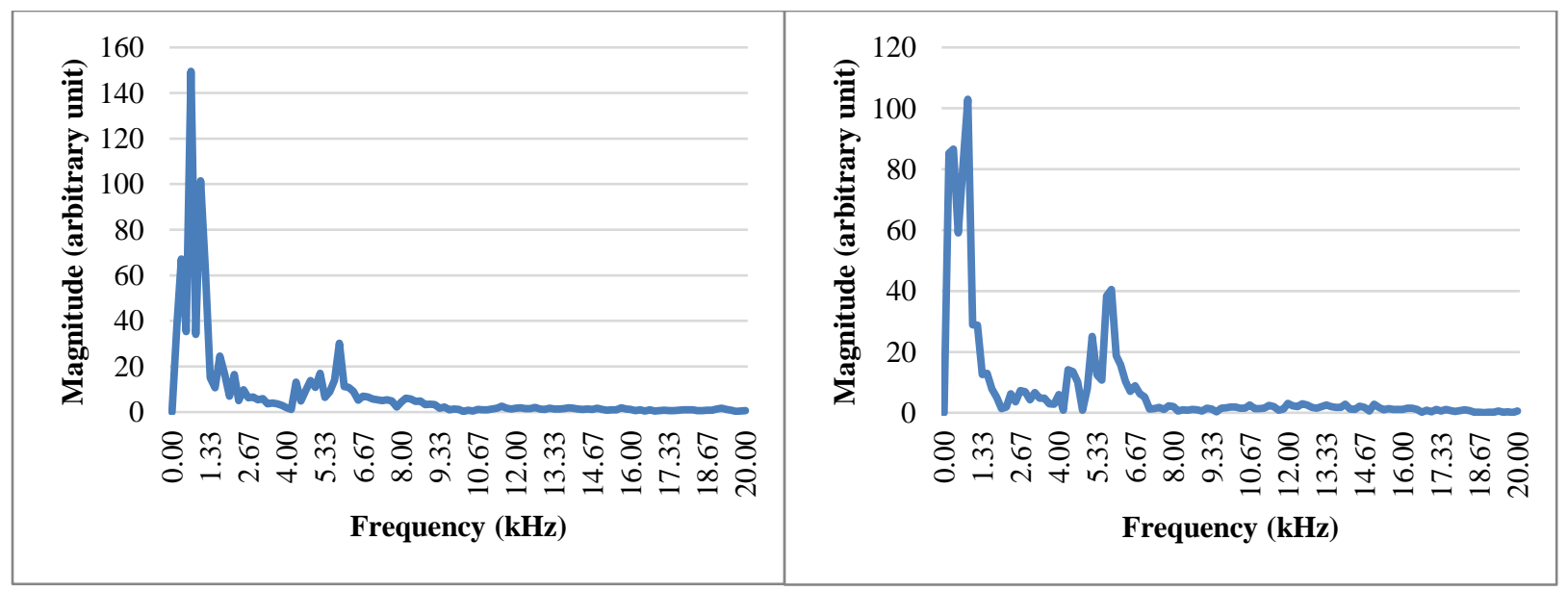

(a) MIC 1 intact

(b) MIC 2 intact 


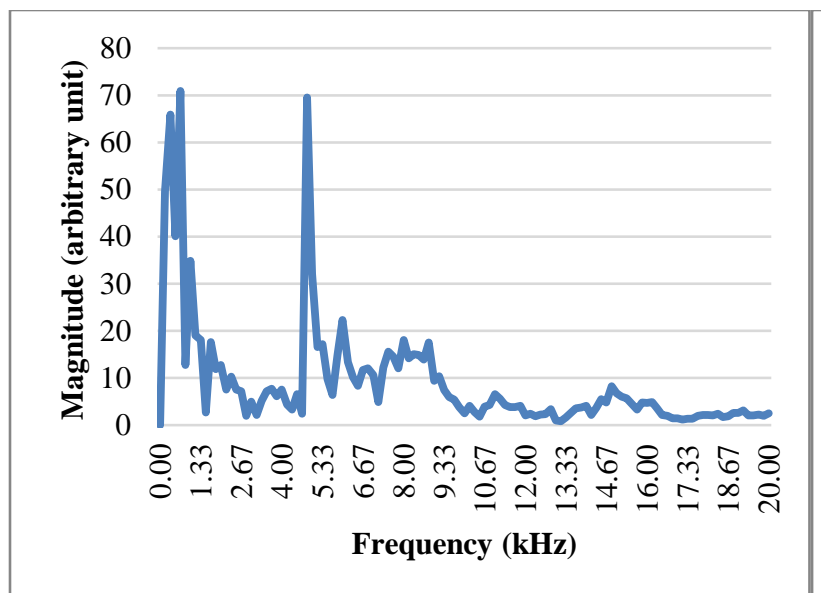

(c) MIC 1 damaged

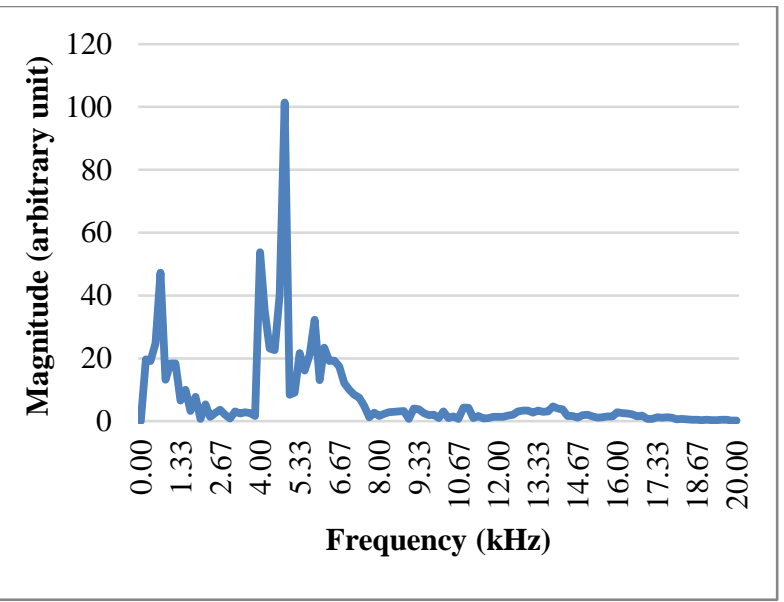

(d) MIC 2 damaged

Figure 13: FFT for intact and damaged points of Test 1

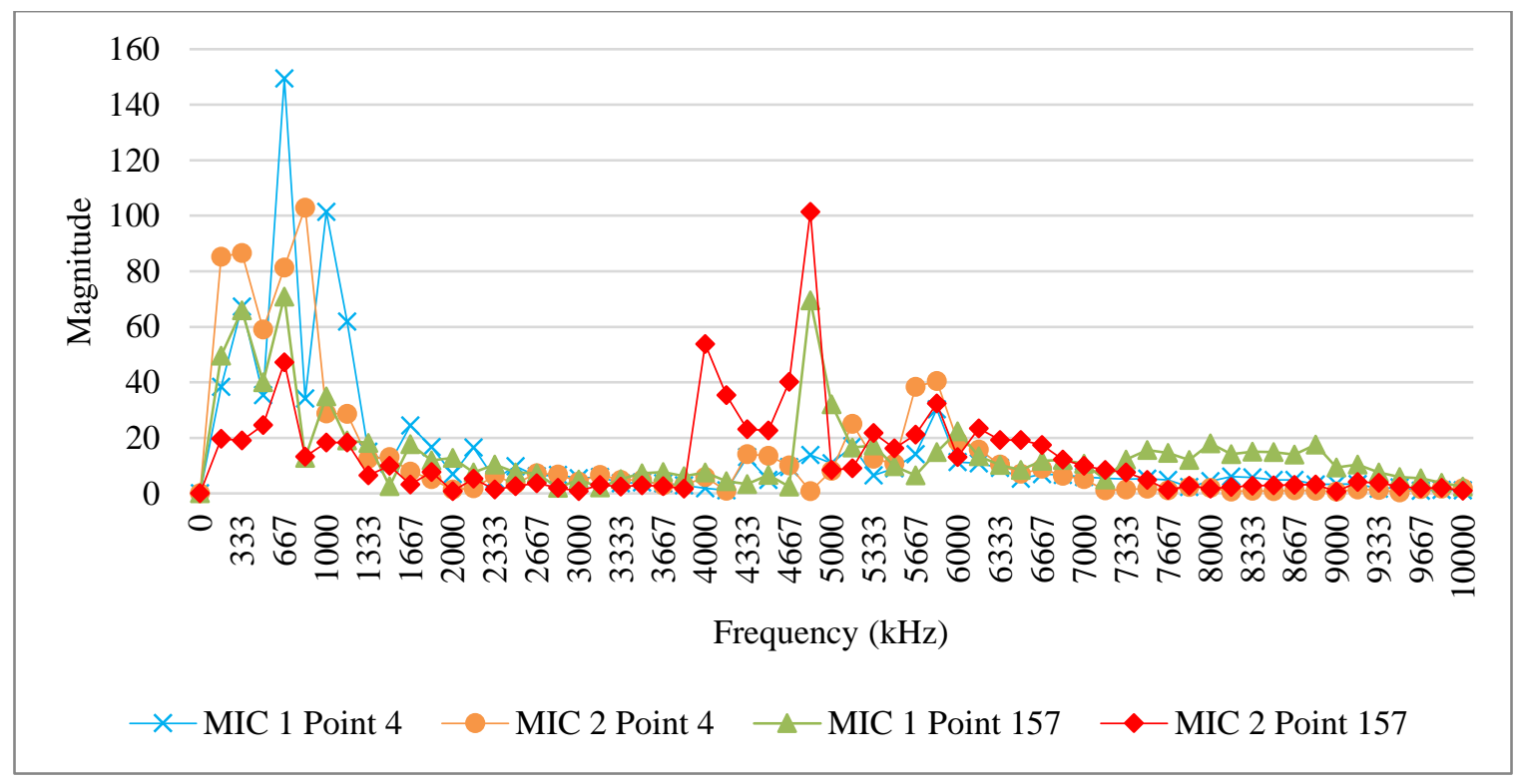

Figure 14: Spectral comparison for test point 4 and 157 from Test 1

There are two dominant peaks observed when damaged locations are analyzed using the FFT observed in Figure 13 and Figure 14 . The lower frequency peak $(<1 \mathrm{kHz})$ was taken as the direct acoustic signal from the impact. The higher frequency peak is a result of the reflection of 
longitudinal waves within the structure, either at a defect or from the edges of the specimen, and the flexural vibration of the structure or location above damaged regions. Intact test locations exhibit a low frequency peak because of a direct acoustic signal, similar to the peak seen at damaged sites. The second peak at the intact site was taken to be a reflection of the bottom of the beam and was present at the damaged locations, but has a much lower magnitude compared to the peak corresponding to damage detection. The intact and damaged locations are distinguished by the peak caused by the reflection of the longitudinal wave off the internal damage, which was not observed at the intact location. While the unit of acquisition was voltage, the graphical representation is taken as an arbitrary unit, as it is used mainly to compare results, and the normalization of the signal causes the signal to be unit-less.

After the spectral data are collected, cutoff values must be determined based on known information about the damage location of the structure and sensor spacing. The strength of the spectral response was found to vary between the responses from MIC 1 and MIC 2 for Test 1 because of the varying distance from the impact site. This resulted in the use of different cutoff criteria for the magnitude of the spectral response from each microphone. The cutoff value sets the threshold for definition of a damaged or intact region of the structure between the impact site and the microphone. An inherent problem of the technique, however, stems from the use of cutoff criteria of spectral results, as each signal contains information in the range of interest. The cutoff values used must be refined to accurately represent the state of the structure. This poses a problem when the state of the structure is unknown at the time of testing. One possible solution when investigating RC structures is to use chain dragging or sounding to audibly distinguish several damaged and intact regions so that signals gathered from those locations can be classified accordingly and used as references for the rest of the data. 
Figure 15 gives a damage map of the beam using several different cutoffs for both MIC 1 and 2 with the sensor configuration for Test 1 . The areas in yellow are defined as intact and the areas in red and orange are defined as damaged. The colour scale defined in MATLAB® was accentuated for clarity by setting the value of data points below the cutoff value to zero. This increases the contrast of the intact and damaged areas and allows the areas identified as damaged to be clearly visible. The general equation for the classification of the spectral data found using FFT was found from Equation 25:

$$
\begin{aligned}
& \text { Maximum spectral amplitude }(4-7 \mathrm{kHz})= \\
& 0, \text { amplitude }<\text { cutoff } \\
& \left\{\begin{array}{r}
\text { amplitude, amplitude } \geq \text { cutoff } \\
100, \text { amplitude } \geq 100
\end{array}\right.
\end{aligned}
$$

Given the knowledge of damage locations in the specimen used in this study, the data collected from MIC 1 has better agreement with the expected damage locations with a cutoff value of 65 as shown in Figure 15(d). Data collected from MIC 2 has better agreement with the expected damage locations with a cutoff value of 55-60, although in general the results from signals collected from MIC 2 perform poorly. Even with the optimized cutoff value, data from both microphones have several areas where the FFT data analysis gives false positives for damage identification based on in-situ damages.

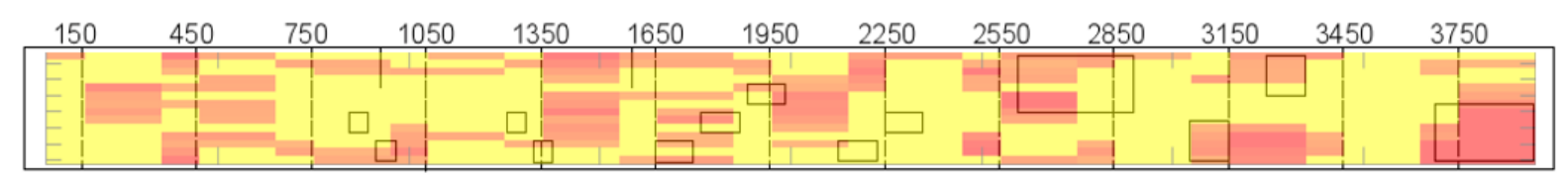

(a) $55-55$

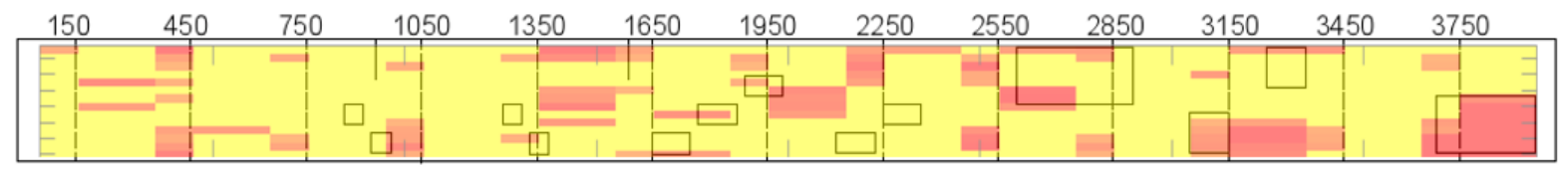

(b) $55-70$ 


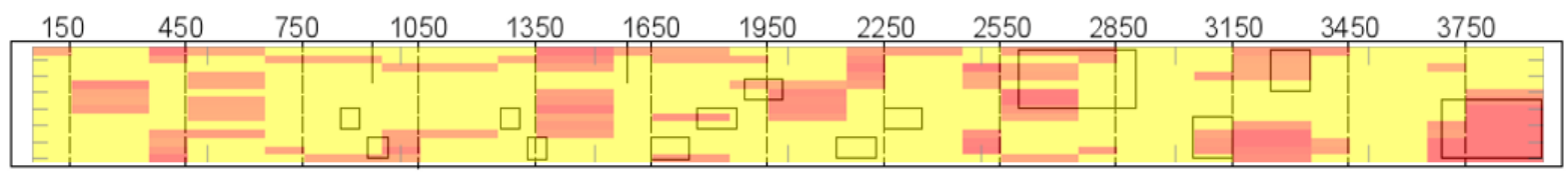

(c) $60-60$

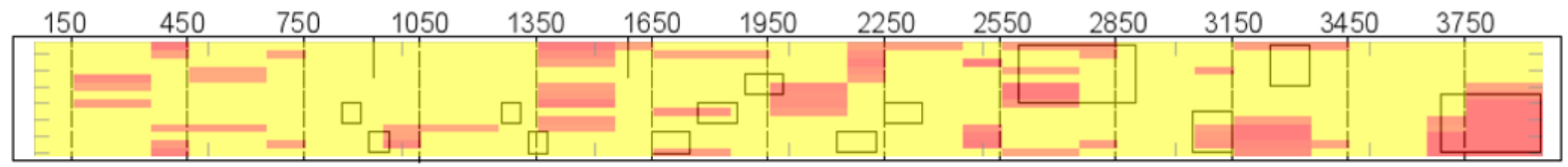

(d) $65-65$

Figure 15: Imaging of damage using FFT for Test 1 data (MIC 1 cutoff value - MIC 2 cutoff value)

The same method of FFT analysis was carried out for Test 2. It is seen from Figure 16 that the ability of FFT analysis to correctly identify in-situ damage diminishes with increased sensor spacing from the point of excitation. The instances of large regions of false positives are particularly troublesome because the repercussions these findings can have on maintenance and safety decisions. Further, the smaller damages, overall, were not detected. This was expected due to their positioning and the size of the damages $(50-100 \mathrm{~mm})$ relative to the spacing of the sensors from the point of excitation [62]. The reflected wave from the edges of the specimen was part of the spectral data collected as well, and may create false identification of damage. Wavelet transformation has therefore been employed to analyze the data because wavelet transforms can analyze spectral information without losing temporal information and have the added benefit of filtering properties.

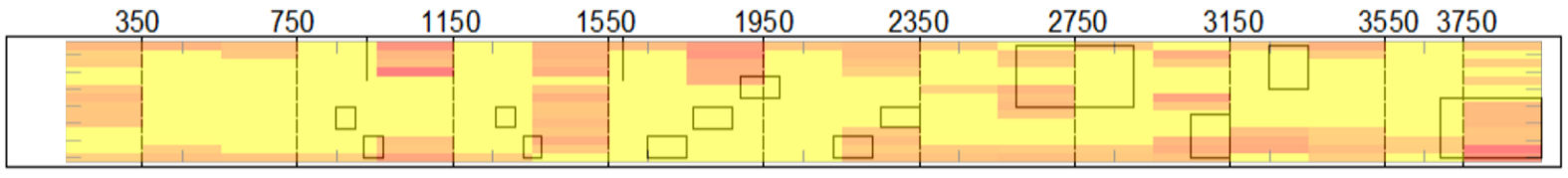

(a) $38-38$ 


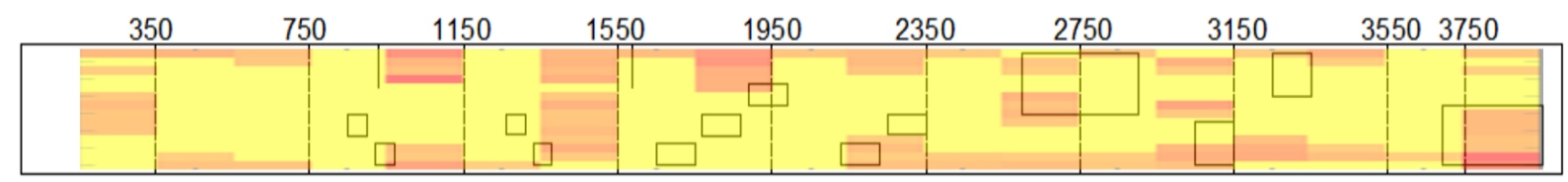

(b) $40-40$

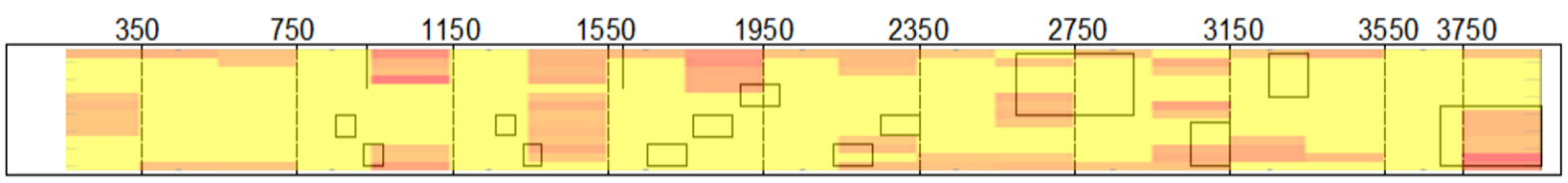

(c) $42-42$

Figure 16: Imaging of damage using FFT and cutoff criteria for Test 2 (MIC 1 cutoff MIC 2 cutoff)

The results indicate that the small damages and cracks were not readily identified simply using the FFT analysis. The large near surface damages were identified but resolution is lost in an attempt to limit the false positives. Wavelet transforms will subsequently be used in an attempt to improve upon the results found using the FFT method.

\subsubsection{Wavelet Transform Analysis}

Wavelet transforms were used to decompose the signal as an alternative to the FFT in an attempt to better isolate pertinent spectral data corresponding to damages in the structure. Wavelet scales, discussed in Section 2.4.1, were used to dilate or compress the wavelet form in order to better observe certain spectral characteristics of the signal. Figure 17 provides an indication as to the relationship between wavelet scale and the frequency that is most readily identified by that scale. Figure 18 presents a focused view of the scale-to-frequency relationship for three of the wavelets studied. 


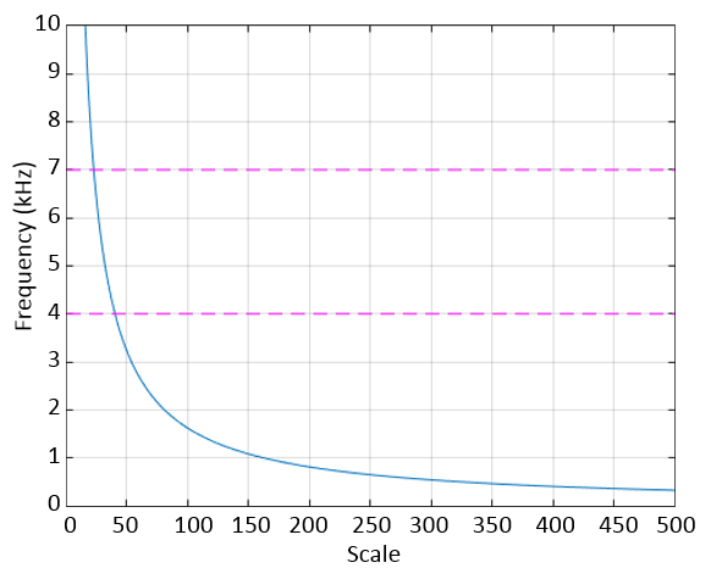

Figure 17: Corresponding frequency and scales for the Morlet wavelet

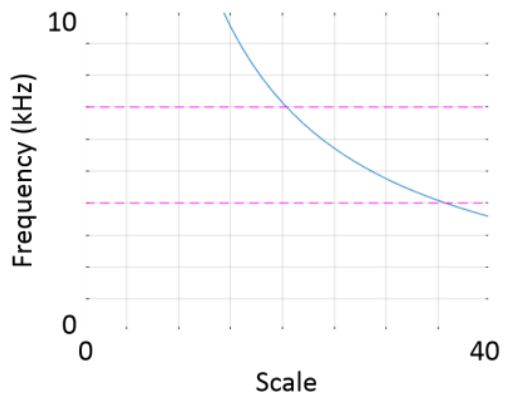

(a) DB 4

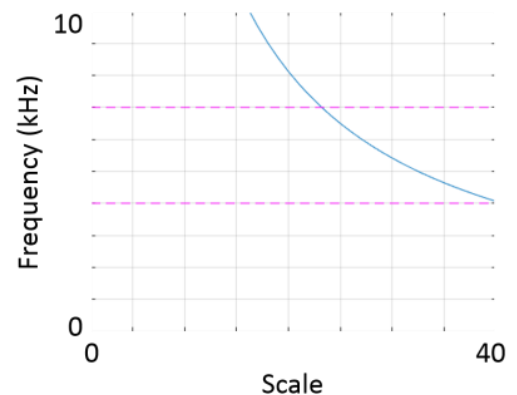

(b) Morlet

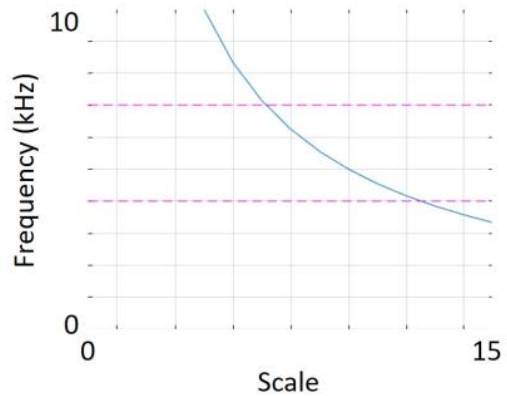

(c) Mexican Hat

Figure 18: Corresponding frequency and scales of wavelets

It follows that the scale range of interest is below 40 for the Morlet wavelet, and is, in fact, the range where the spectral information for the damage sensitive feature was found for all five of the analyzed wavelets. Figure 19 shows the Morlet wavelet at various scales for damaged point 157. The maximum coefficient value occurs using a scale of 21 . 


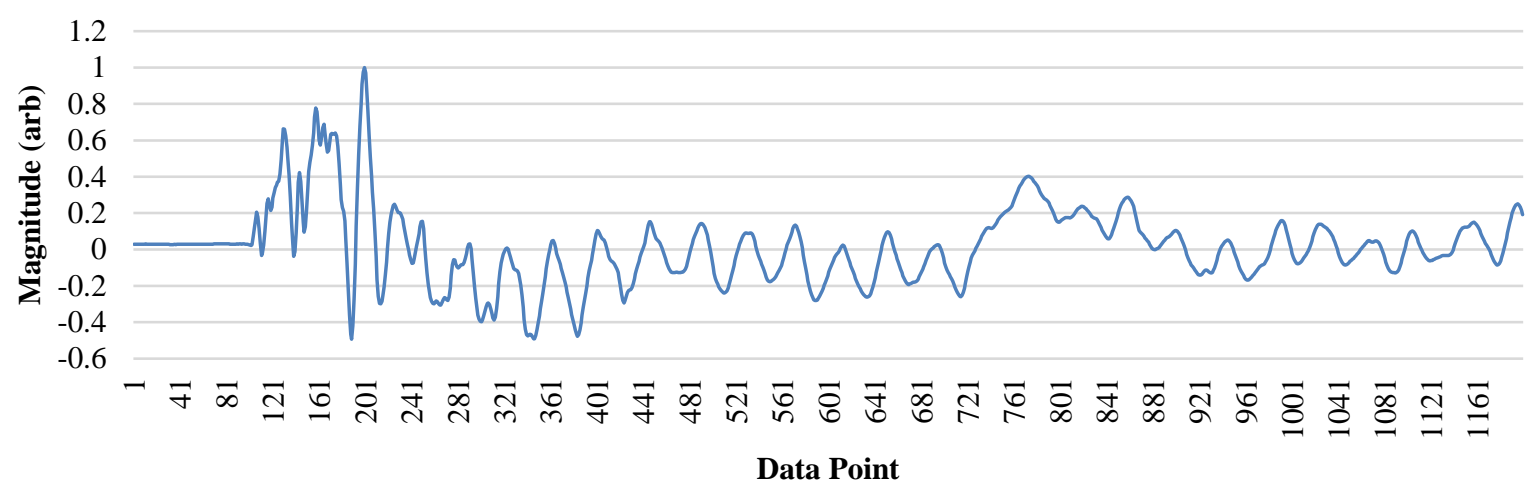

(a) Recorded signal

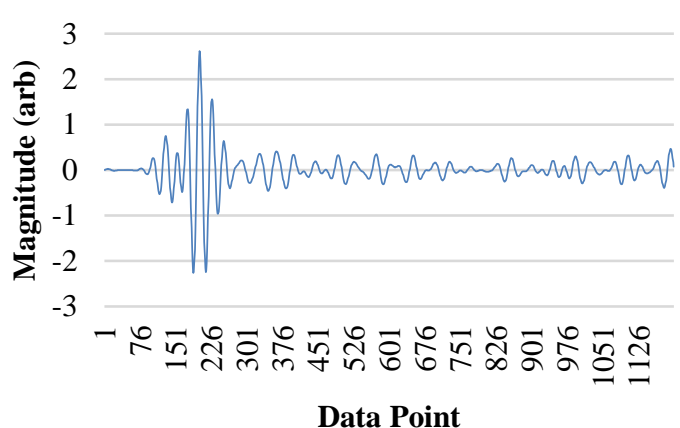

(b) Wavelet scale 21

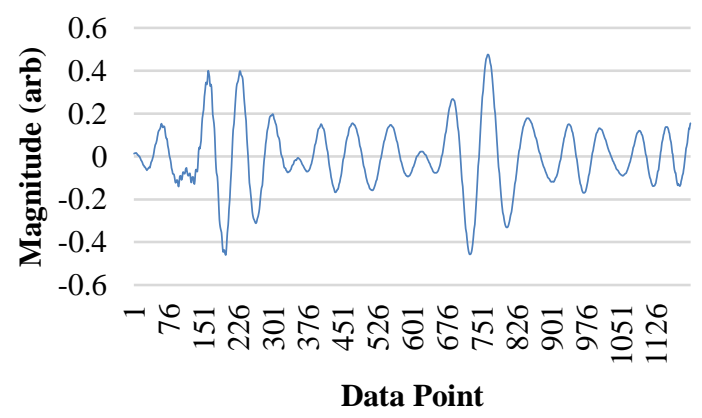

(d) Wavelet scale 27.5

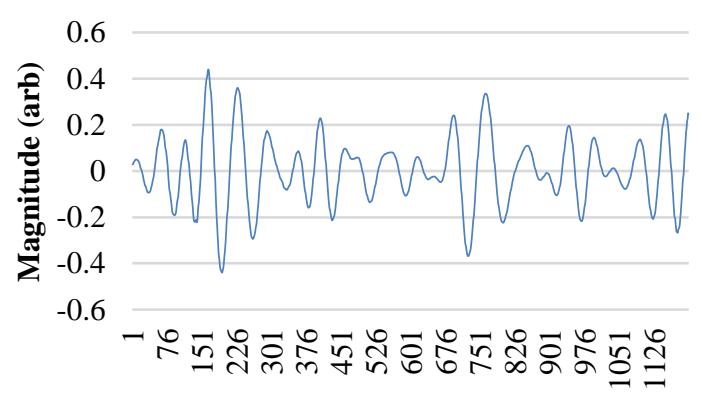

Data Point

(c) Wavelet scale 25

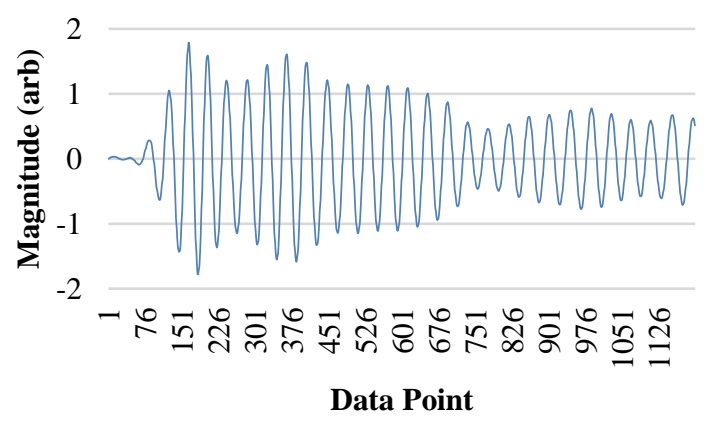

(e) Wavelet scale 34

Figure 19: Morlet wavelet transform for Point 157 MIC 1 Test 1

\subsubsection{Relative Energy of Coefficients Produced from Wavelet Transforms}

A comparison of the percentage of energy obtained from the use of different scales of the same mother wavelet was used as a damage sensitive feature to produce a damage distribution within the RC beam. A broad scalogram was used to look at the structure of the signal over a range of 
wavelet scales and to confirm the region of interest. Figure 20 shows three of the wavelets used and the percentage of energy of each coefficient relative to all of the coefficients in the specific scalogram.
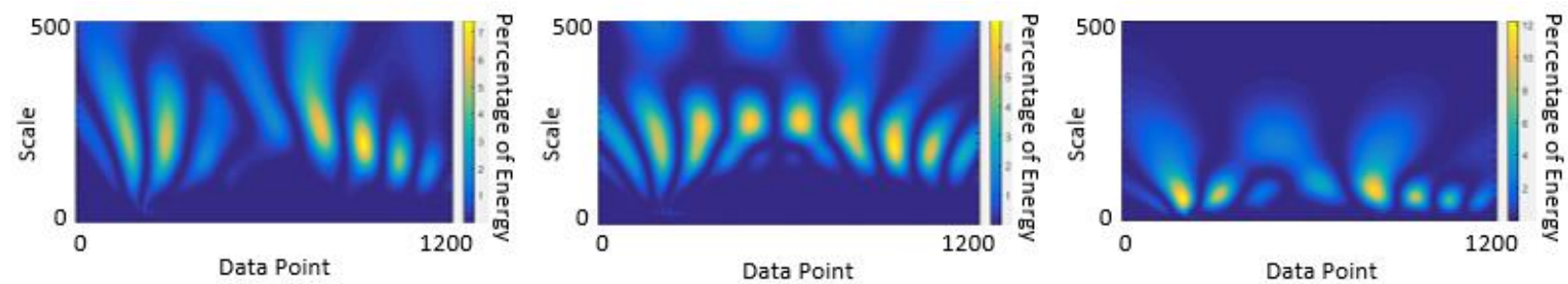

(a) MIC 1 point 4 (intact)
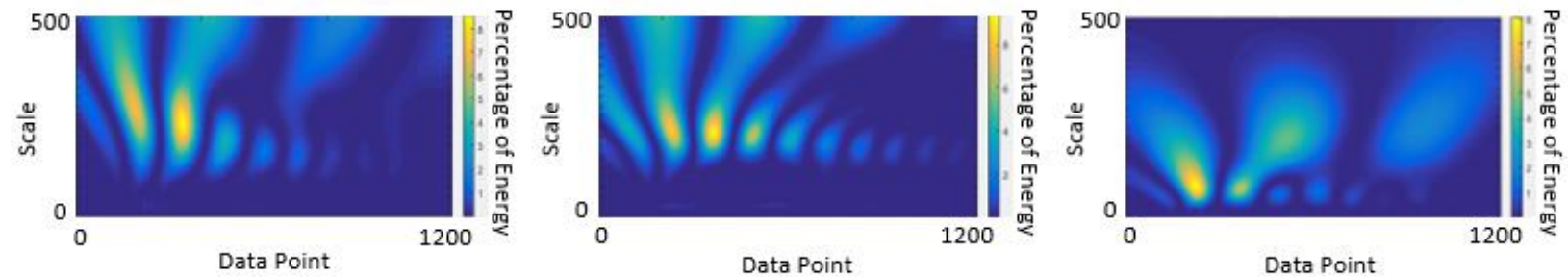

(b) MIC 2 point 4 (intact)
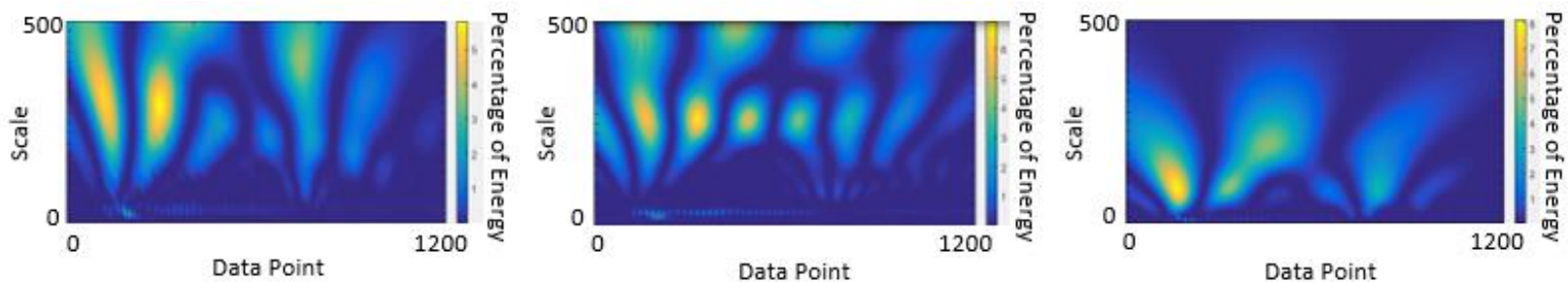

(c) MIC 1 point 157 (damaged)
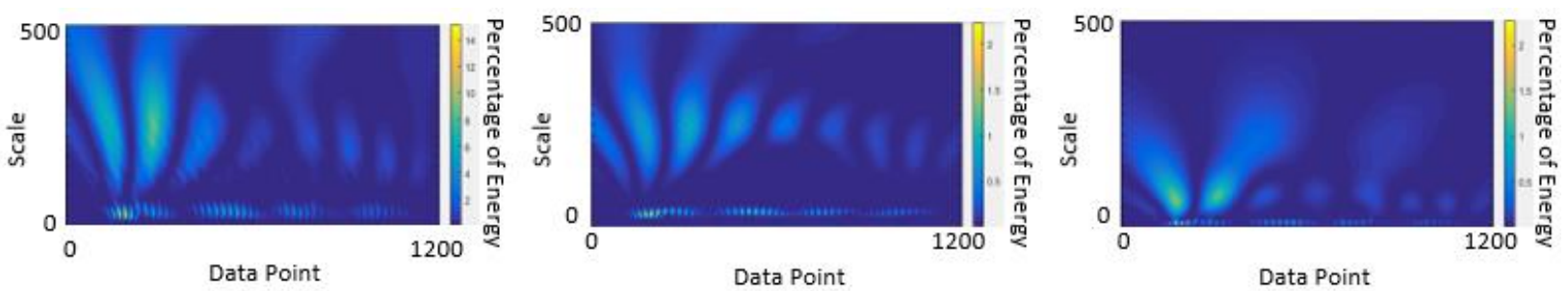

(c) MIC 2 point 157 (damaged)

Figure 20: DB 4 (left), Morlet (center), and Mexican Hat (right) wavelet scalogram for scales 0.5:0.5:500 
It is evident from Figure 20 that the percentage of energy increases in the low end of the scale range between point 4 and point 157 . This acts as the part of the damage sensitive feature of the wavelet transforms, akin to the magnitude increase above $4 \mathrm{kHz}$ of the FFT at damaged locations, and is consistent with the expected range from Figure 17 and Figure 18.

Initially, the percentage of energy value as a means of damage detection was used from the band corresponding to the frequency range of interest only. The strength of the percentage of energy approach, however, comes from the ability of the method to present the results from each scale with respect to information from other scales. Equation 26 gives the value at each point in the scalogram:

$$
\text { Percentage of energy } y_{i, j}=\frac{100 \times C_{i, j}^{2}}{\sum_{1}^{n} \sum_{1}^{m} C_{n, m}^{2}}
$$

where $C_{i, j}$ is the wavelet coefficient at a discrete data point, $i$, and scale, $j$. The bottom term sums the wavelet coefficients over every data point, $n$, and scale, $m$. Figure 21 is provided to illustrate the results of wavelet analysis looking at the signal over a broad scale range and at the scale range representing the damage sensitive frequency found from employing the FFT. 

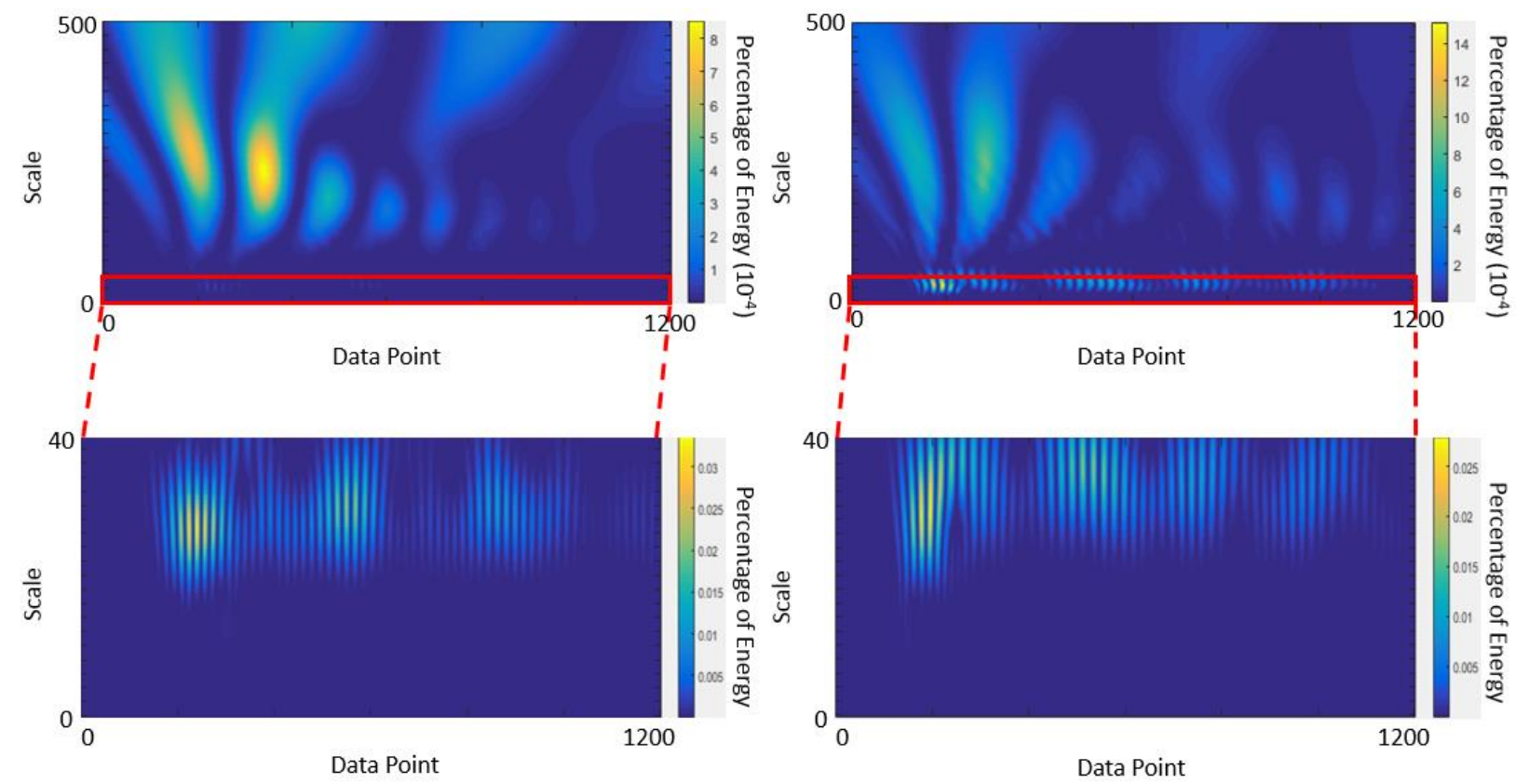

Figure 21: Focused view of DB4 wavelet with a scale range of 0.5:0.5:40 for MIC 2 point 4 (left) and MIC 2 point 157 (right) Test 1

By analyzing images like those in Figure 21, it was apparent that there are two bands of interest for the data gathered. The maximum value of the percentage of energy was extracted from each band and the value from the damage sensitive band was divided by the maximum in the second band to create an influence factor noted in Equation 27:

$$
\text { Energy impact factor }=\frac{\text { Maximum percent of energy band } 1}{\text { Maximum percent of energy band } 2}
$$

The two bands of scales were developed, as mentioned, using the scale-to-frequency relationship observed in Figure 18. Band 1 contains high frequency information in the damage sensitive range defined previously, and band 2 contains low frequency information. For the DB4 wavelet, the damage sensitive band was taken from a scale range of 20-35, and the band corresponding to lower frequencies was taken from a scale range of 36-300. The scale value of 300 was chosen for analysis as the corresponding frequency changed very little from the scale 
300 to the scale 500 and the maximum percentage of energy value for the second band fell below the scale of 300 . Further, the scale was capped at 300 as a means of limiting computational cost.

Once the energy impact factor (EIF) was calculated, it was filtered using a cutoff value to distinguish between damaged and intact data. The analysis process for building $\mathrm{C}$-scans using the EIF is provided in Figure 22.

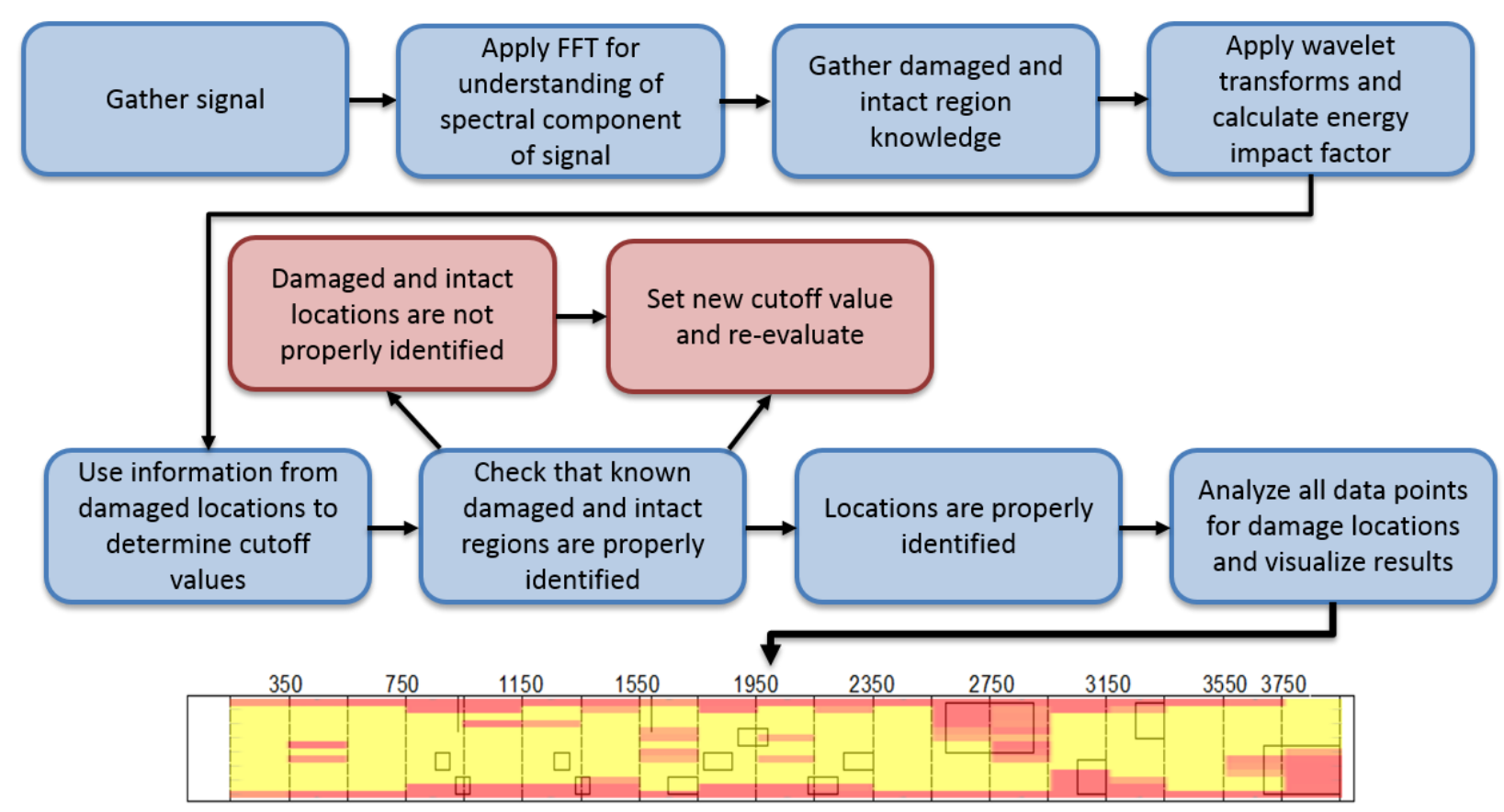

Figure 22: Flow chart of EIF implementation to visualize internal structural state

The use of the EIF for Test 1 analysis gives good resolution of the larger damages and has far fewer false positives for damage than the FFT results. Specifically, using the Mexican Hat wavelet to analyze the data produced 40 false positives compared to 60 from the FFT analysis. All five wavelets perform similarly, although the Mexican Hat wavelet has the fewest erroneous results as shown in Figure 13. The results were filtered in a similar way to the FFT results, although the ratio found was biased by a factor of ten to increase the contrast in the image of damaged and intact regions. A cutoff value was used in order to distinguish damaged 
areas from intact areas. These cutoff values are found in Table 4, along with the scale range for band 1 used to compute the EIF. Equation 28 was used for the classification of the EIF as representing a damaged or intact location:

$$
\begin{aligned}
\text { Energy impact factor }= \\
0, \text { Energy impact factor }<\text { cutoff } \\
\qquad \begin{aligned}
\text { Energy impact factor } \times 10, \text { Energy impact factor } & \geq \text { cutoff } \\
10, \text { Energy impact factor } & \geq 10
\end{aligned}
\end{aligned}
$$

Equation 28

The large in-situ damages $(>100 \mathrm{~mm})$ are located well by the impact energy factor method in both Tests 1 and 2. This was expected as the depth of the damage $(50 \mathrm{~mm})$ was suitable for the frequency range of the microphones and the size of the damages was roughly equal to the spacing of the sensors. The smaller damages, damages below $50 \mathrm{~mm}$ depth in the specimen, and cracks, were not well identified. This may have been caused by movement of the damages during casting, or in the case of damages below $50 \mathrm{~mm}$, the locating frequency may be higher than the microphones are able to detect. Cracks are more readily discovered using shear waves that are not collected by non-contact sensors as discussed previously in Section 2.10.3. The smaller damages may also be difficult to identify when the sensor spacing is larger than the damage because of a smaller reflection of the waves, a less prominent flexural vibration response created by the damage, and the microphone collects less relative energy from the frequency range of interest.

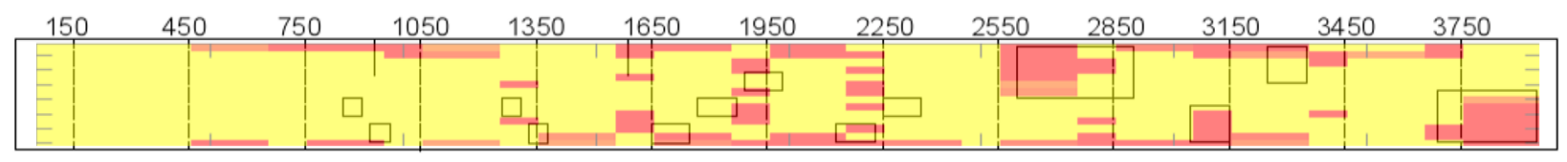

(a) DB 4 wavelet 


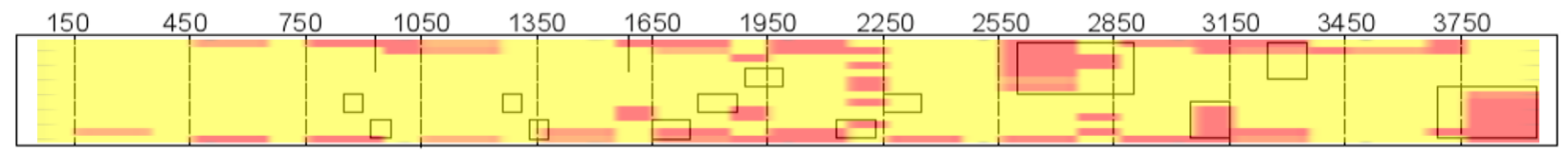

(b) DB 6 wavelet

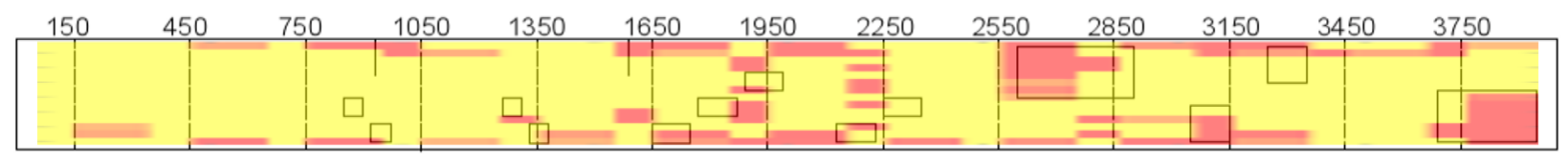

(c) DB 7 wavelet

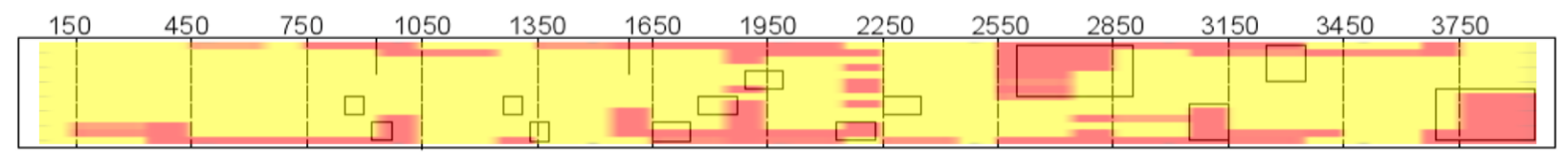

(d) Morlet wavelet

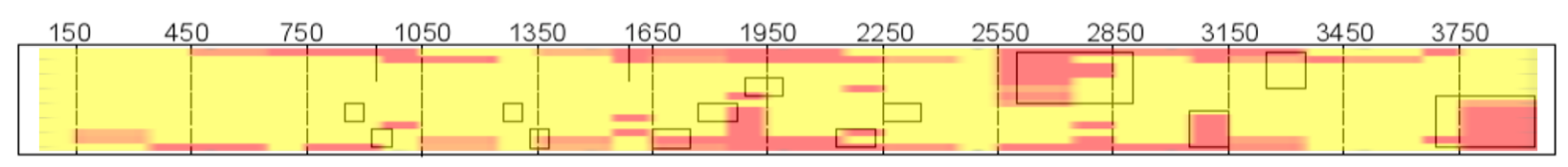

(e) Mexican Hat wavelet

Figure 23: Imaging of damage using EIF from wavelet transforms for Test 1

Table 4: Scale and cutoff range for EIF using wavelet transforms Test 1

\begin{tabular}{cccc}
\hline Wavelet & $\begin{array}{c}\text { MIC 1 and 2 Damage } \\
\text { Sensitive Scale }\end{array}$ & MIC 1 Cutoff (-) & MIC 2 Cutoff (-) \\
\hline DB 4 & $20: 35$ & 1.7 & 0.55 \\
DB 6 & $21: 36$ & 2.2 & 0.5 \\
DB 7 & $19: 34$ & 2 & 0.5 \\
Morlet & $22: 40$ & 1.9 & 0.6 \\
Mexican Hat & $7: 12$ & 1.9 & 0.5 \\
\hline
\end{tabular}

Test 2 gives similar results to Test 1, especially when identifying the larger damages. The results from Test 2 continue to show a propensity for erroneous results along the first two rows of each the top and bottom edge of the specimen with a microphone spacing of $200 \mathrm{~mm}$. These false positives occur because the reflection of the longitudinal waves reflects off the concrete-air interface at the edges in the same way they reflect at an internal delamination or void. The use of this technique on a bridge slab would have fewer false positives from this effect because of the 
increased surface area to perimeter ratio of the bridge deck compared to the specimen used in this study. The results and scale information for Test 2 are seen in Figure 24 and Table 5, respectively. The large damages are readily identified and the false positives are limited to the edge of the beam in most cases. The same cutoff values of MIC 2 in Test 1 were used in Test 2. The results show good replicability, consistent discovery of the large damages, and far fewer instances of false positives for damage than the FFT results presented in Figure 16 in Section 3.4.1. For example, the comparison was quantified using the Mexican Hat wavelet analysis. The wavelet analysis yielded 39 false positives compared to 55 for the FFT analysis of Test 2 .

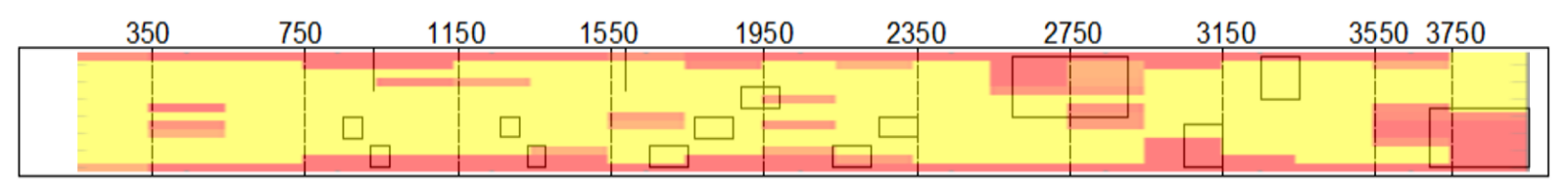

(a) DB 4 wavelet

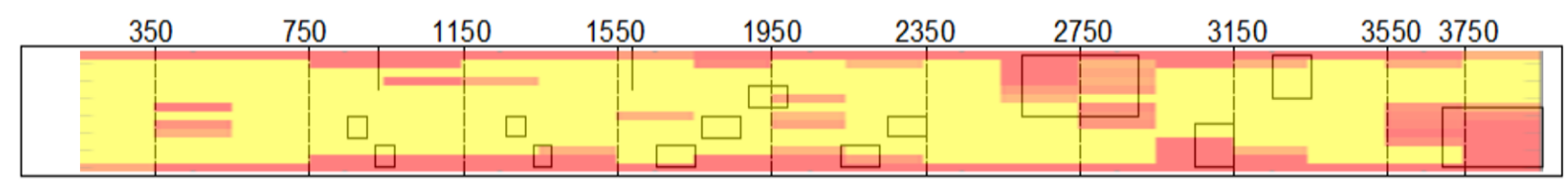

(b) DB 6 wavelet

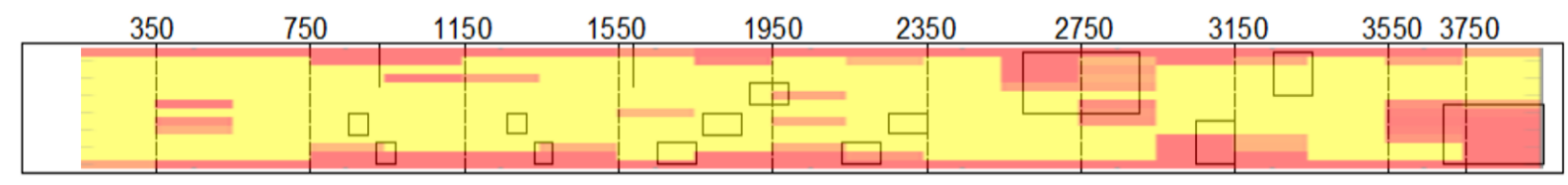

(c) DB 7 wavelet

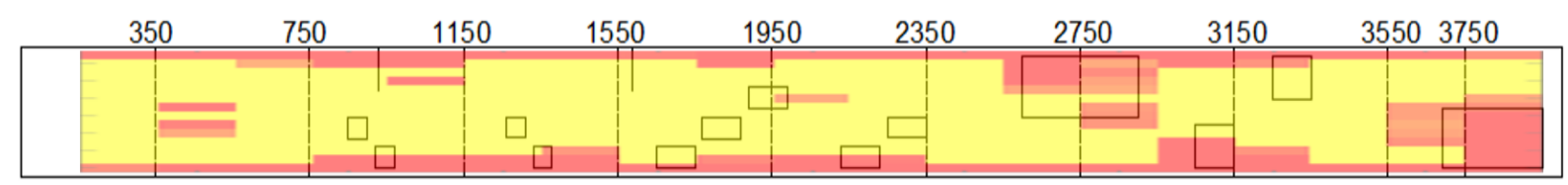

(d) Morlet wavelet

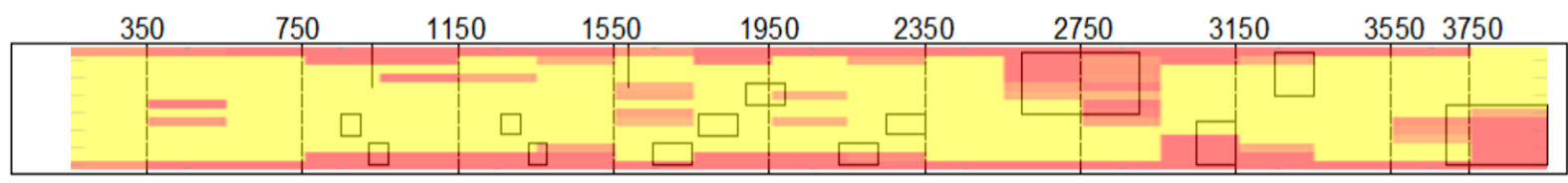

(e) Mexican Hat wavelet

Figure 24: Imaging of damage using EIF from wavelet transforms for Test 2 
The wavelet transform results also do not clearly indicate the small damages in the center and left portion of the beam or the two in-situ cracks. The largest damages are clearly identified and the $100 \times 100 \mathrm{~mm}$ void was also found in both Test 1 and Test 2 . This is expected due to the sensor spacing and it is expected that closer spacing would enable the method to be used for smaller damages.

Table 5: Scale and cutoff range for EIF using wavelet transforms Test 2

\begin{tabular}{ccc}
\hline Wavelet & $\begin{array}{c}\text { MIC 1 and 2 Damage } \\
\text { Sensitive Scale }\end{array}$ & $\begin{array}{c}\text { MIC 1 and 2 Cutoff } \\
(-)\end{array}$ \\
\hline DB 4 & $20: 35$ & 0.55 \\
DB 6 & $21: 36$ & 0.5 \\
DB 7 & $19: 34$ & 0.5 \\
Morlet & $22: 40$ & 0.6 \\
Mexican Hat & $7: 12$ & 0.5 \\
\hline
\end{tabular}

\subsection{Comparison of Performance Using FFT and Wavelet Transforms}

The comparison of the FFT and wavelet transforms uncovers several interesting features. Figure 25-Figure 29 compare counts of true positives and F1 scores from different areas of the two tests, allowing the strengths and weaknesses of each method to be observed. The large damage region was taken as the area from $2750 \mathrm{~mm}$ to the right hand edge of the structure in all cases. 


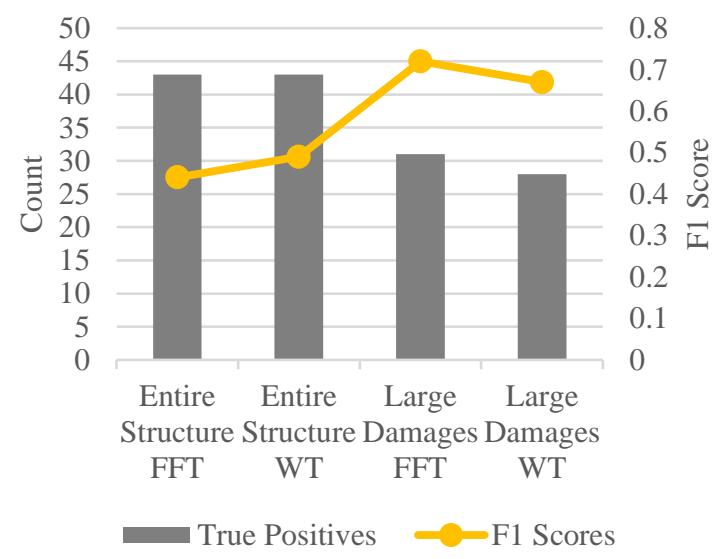

(a)

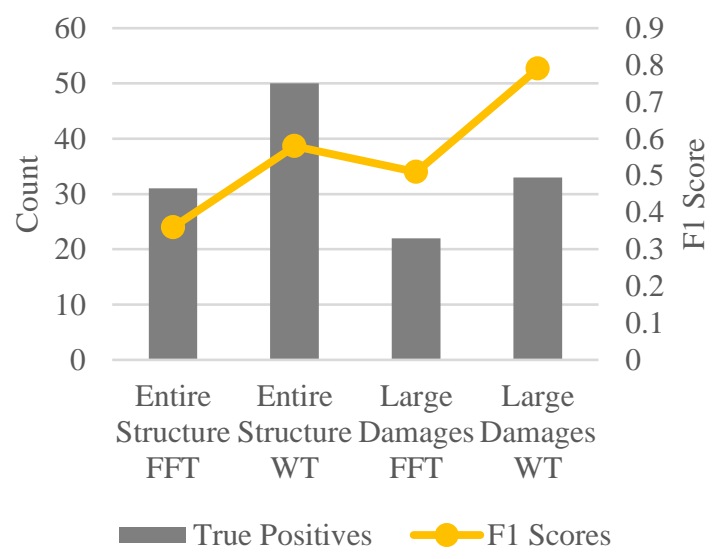

(b)

Figure 25: Comparison of performance between FFT and Mexican Hat wavelet analysis for (a) Test 1 and (b) Test 2

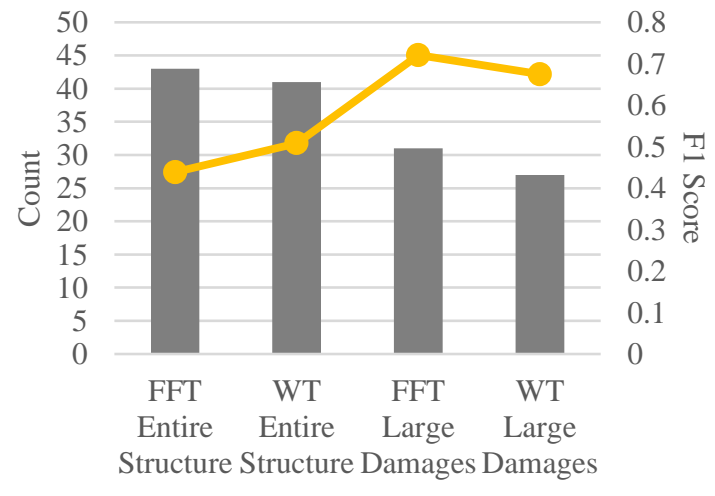

(a)

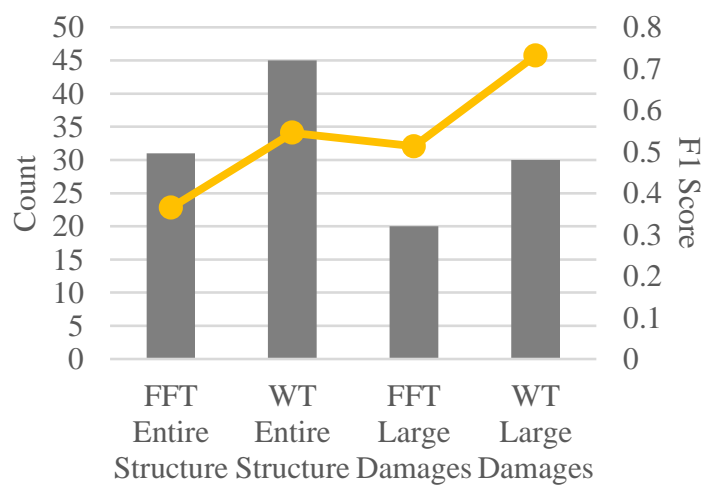

(b)

Figure 26: Comparison of performance between FFT and DB4 wavelet analysis for (a) Test 1 and (b) Test 2 


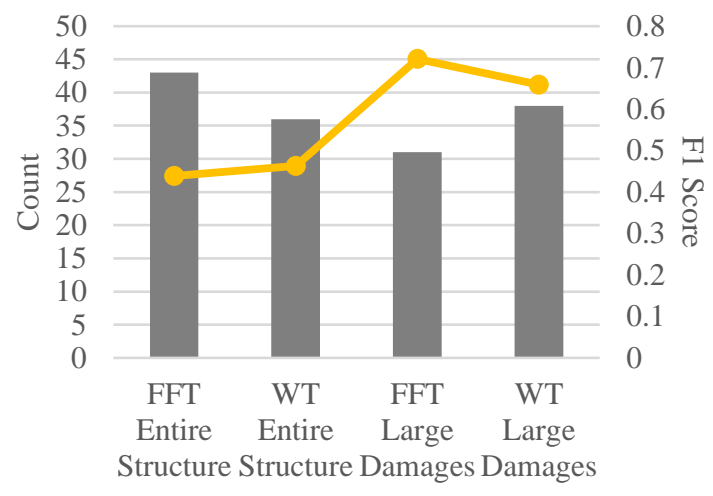

True Positives $\quad-$ F1 Score

(a)

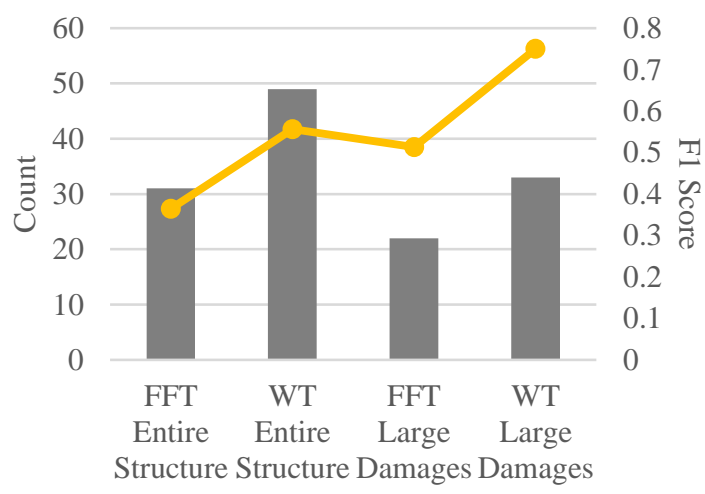

True Positives $\longrightarrow$ F1 Score

(b)

Figure 27: Comparison of performance between FFT and DB6 wavelet analysis for (a) Test 1 and (b) Test 2

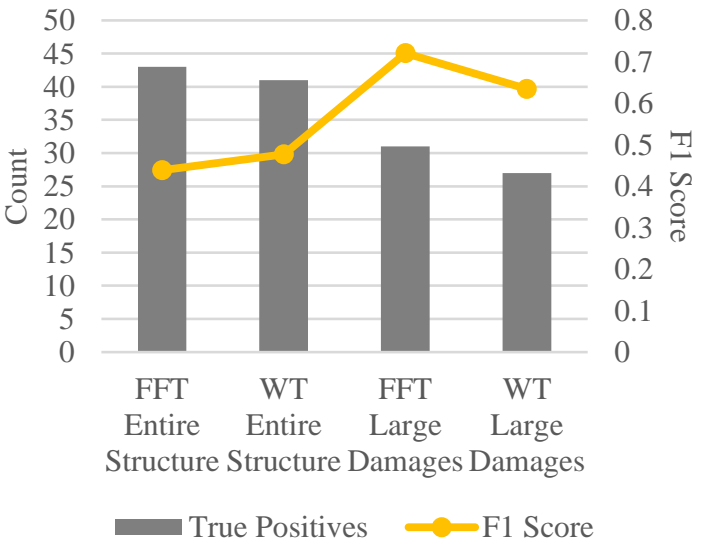

(a)

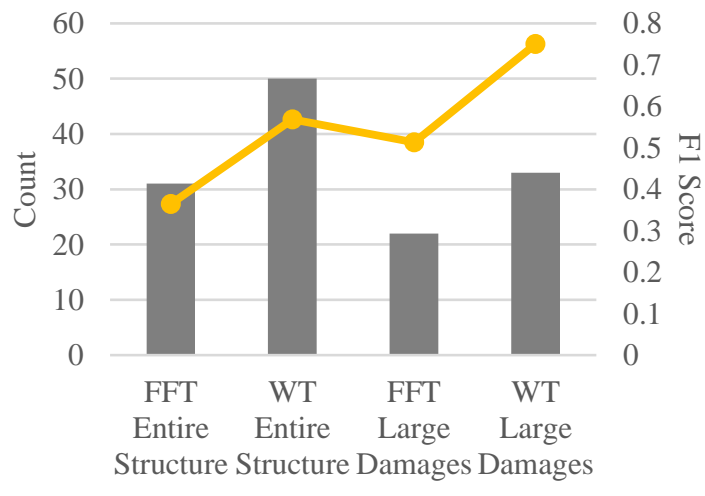

True Positives $\quad$ F1 Score

Figure 28: Comparison of performance between FFT and DB7 wavelet analysis for (a) Test 1 and (b) Test 2 


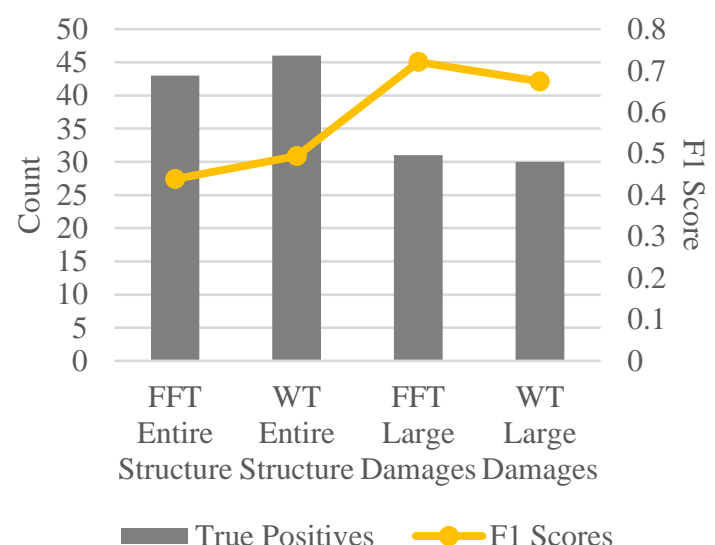

(a)

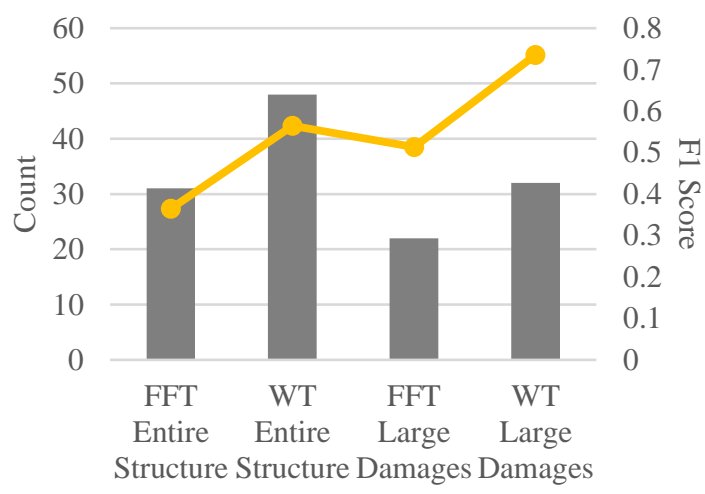

True Positives $\longrightarrow$ F1 Scores

(b)

Figure 29: Comparison of performance between FFT and Morlet wavelet analysis for (a) Test 1 and (b) Test 2

Table 6: F1 score comparison for full lab specimen

\begin{tabular}{|c|c|c|c|}
\hline Test & FFT F1 Score & $\begin{array}{c}\text { Wavelet Transform F1 } \\
\text { Score }\end{array}$ & Wavelet Type \\
\hline 1 & 0.44 & 0.46 & \multirow{2}{*}{ DB4 } \\
\hline 2 & 0.36 & 0.54 & \\
\hline 1 & 0.44 & 0.49 & \multirow{2}{*}{ DB6 } \\
\hline 2 & 0.36 & 0.56 & \\
\hline 1 & 0.44 & 0.47 & \multirow{2}{*}{ DB7 } \\
\hline 2 & 0.36 & 0.57 & \\
\hline 1 & 0.44 & 0.49 & \multirow{2}{*}{ Morlet } \\
\hline 2 & 0.36 & 0.56 & \\
\hline 1 & 0.44 & 0.49 & \multirow{2}{*}{ Mexican Hat } \\
\hline 2 & 0.36 & 0.58 & \\
\hline
\end{tabular}

Figure 25-Figure 29 show that Test 1 has similar results for both methods, which was in part caused by the FFT performing poorly for MIC 2 and the wavelet transforms producing false positives along the transverse edge of the specimen for MIC 2 data. The false positives were reduced by the use of wavelet transforms from 60 to 40 and from 55 to 39 for the entire structure by use of wavelet transforms for Test 1 and 2, respectively. The wavelet transform performs slightly worse than the FFT when the large damages are isolated, with 11 false positives 
compared to the FFTs 10 in Test 1 . The wavelet transform produces 7 fewer false positives than the FFT for the large damages in Test 2, yielding 9 and 16 respectively. The F1 scores, produced from precision and recall of the method, are indicators of the ability of the method to correctly identify damage. The F1 scores from Test 2 show that wavelet transforms far outperform FFT analysis for each case, despite the noted susceptibility of the wavelet transforms to return false positives along the top and bottom edges. It should be noted that the small damages that were included in the statistical analysis as expected positives, although they were not expected to be located. This fact was a contributor to the large damages being inspected independently.

False positives were found along the edge of the specimen from earlier figures of the detected damages. The inability of both methods to determine small damages was also apparent. This could potentially be overcome by closer microphone spacing. It is also possible that the damages shifted during casting, making detection difficult.

\section{FIELD TESTING OF IMPACT-ECHO METHOD}

Testing was carried out at a parking garage where beams showed signs of delamination and corrosion in order to validate the analysis method used in the lab. Destructive testing was not permitted at the site, so a location was chosen where visible markers existed to delineate the damage for validation. Sounding was also used to approximate damaged and intact areas for analysis. The location of the structure created difficult testing conditions with high levels of random ambient noise from the traffic in the area and the parking garage itself, conversation of passerby's, and from the sensor setup because of the spring-loaded impactor. 


\subsection{Experimental Setup}

The experimental setup was adjusted for the orientation of the structure. A rail was used to hang the sensor chassis to avoid the need to hold the microphones and impactor overhead and to guide the chassis. A roller support was used on the opposite side of the rail to prevent vibration upon impact of the beam from the spring-mounted bearing ball. The nominal beam dimension in the parking garage is $356 \times 561 \times 17000 \mathrm{~mm}$, however testing was limited to a 2.8 -meter area around the visibly damaged area on beam B2. The microphones were placed $20 \mathrm{~mm}$ from surface of the concrete and $200 \mathrm{~mm}$ from the source of excitation on either side. The beam was tested at seven rows across the short direction of the beam. Each row was tested at 11 locations in increments of $30 \mathrm{~mm}$ starting $30 \mathrm{~mm}$ from the edge of the beam. Foam was used to dampen the ambient noise and isolate surface waves from the excitation. The sensor setup is visible in Figure 30.

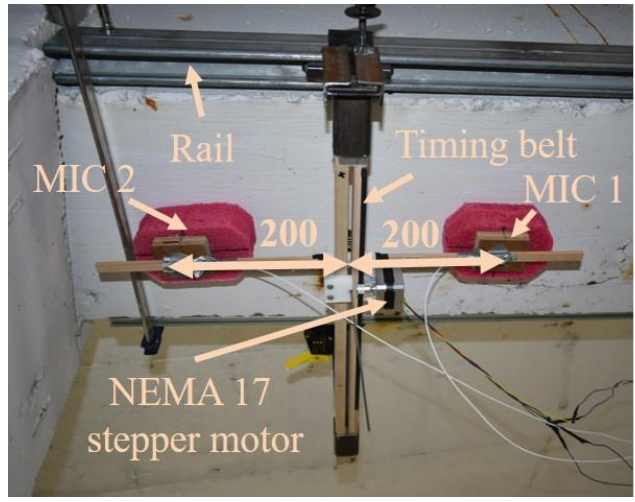

(a)

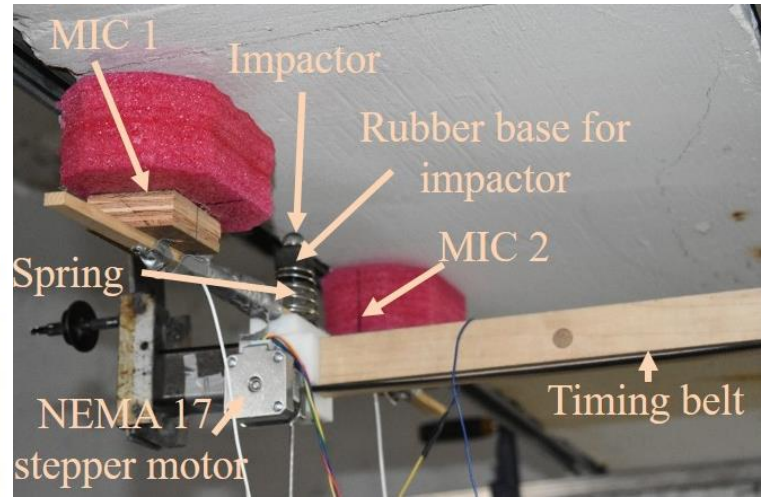

(b)

Figure 30: Parking garage test setup (a) bottom view (b) side view

\subsubsection{Automation of the Experimental Setup}

In order to improve the efficiency and precision of the test setup, a motor was installed to drive the sensor chassis. A NEMA 17 stepper motor was used with a timing belt in order to enable 
precise movement of the impactor and microphones. The motor was used to drive the chassis in $30 \mathrm{~mm}$ increments and then return it to the initial starting point at the end of each row. An Arduino Uno R3 was used with an Easy Driver to power the stepper motor and provide direction and step count. The configuration of the setup is shown in Figure 31.

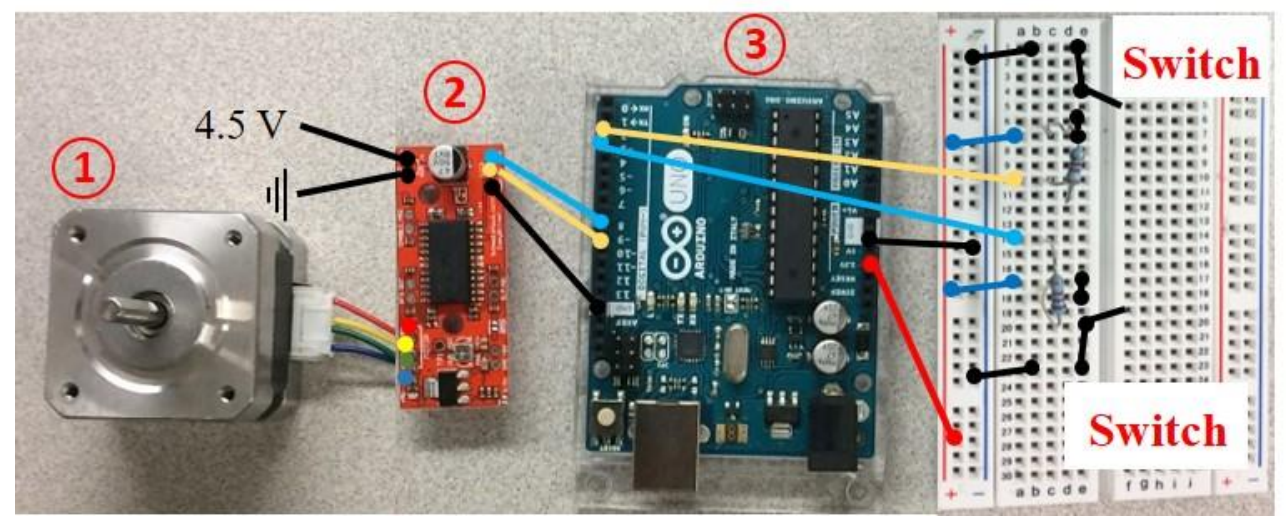

Figure 31: NEMA 17 motor setup

The first object is the NEMA 17 stepper motor, the second is the Easy Driver, and the third is the Arduino Uno R3 board. The configuration in Figure 31 shows the interaction between the modules and was based on an EasyDriver setup [80]. Along with the Arduino Uno R3, motor, and Easy Driver, the setup includes two pull-down resistors in series with the second and third pins of the Arduino board. The circuits with the pull-down resistors keep the second and third pins high unless the switch is closed, connecting the circuit to ground and putting whichever pin the circuit is connected to low. When the second pin goes low, the stepper motor is driven 1200 steps, correlating to $30 \mathrm{~mm}$. When the third pin goes low, the stepper motor goes in the opposite direction 12000 steps, bringing the sensor chassis back to the initial position. The process by which testing was carried out is displayed in Figure 32 . 


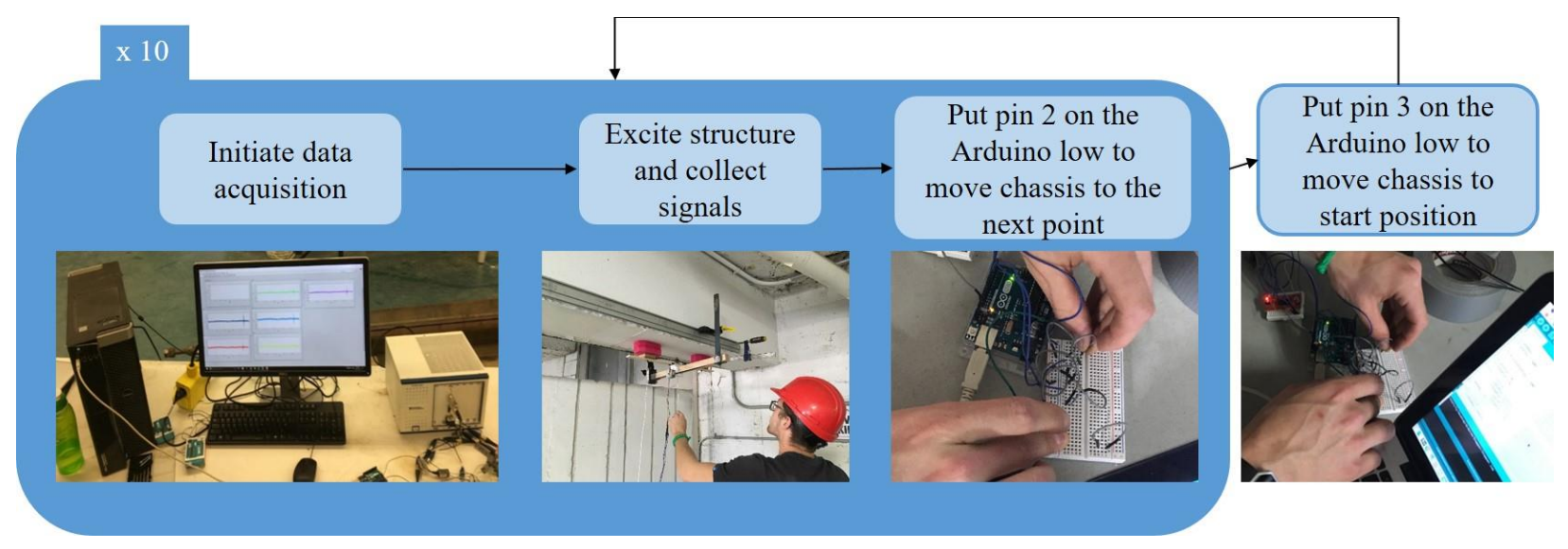

Figure 32: Automated test setup

\subsubsection{Condition of the Structure at Testing}

The beam was visibly damaged at the time of testing. This, along with sounding of the structure using the impactor, allowed for an approximation of damage locations to be made that could be compared to the analyzed results. The state of the beam at each of the rows is seen in Figure 33.

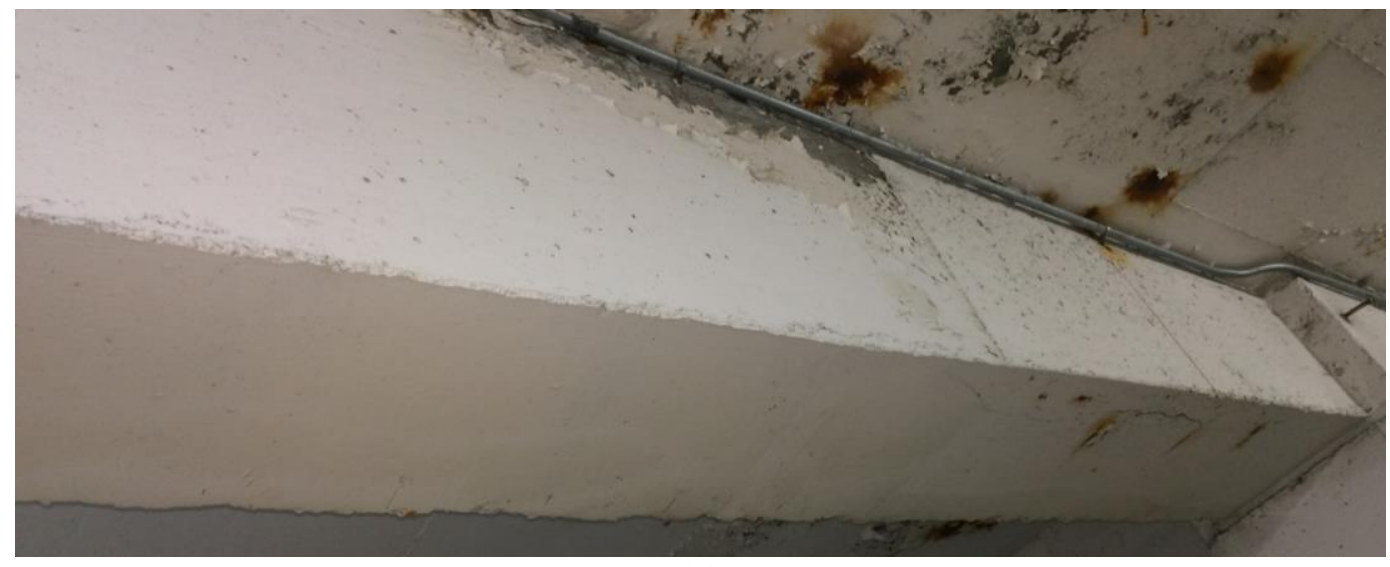

(a)

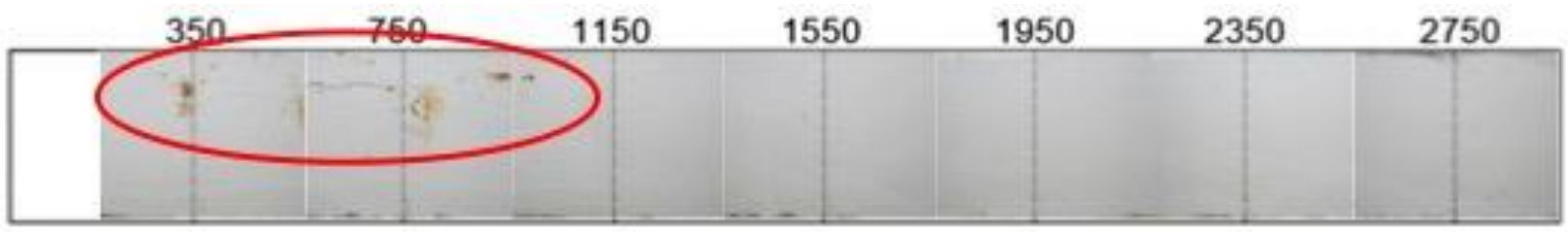

(b) 


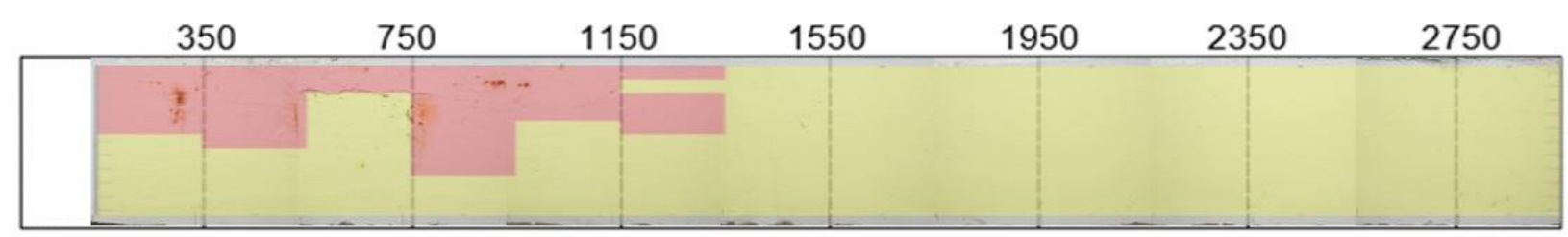

(c)

Figure 33: Condition of beam (a) comprehensive view and (b) compilation of test area images for analysis

Figure 33 (b) is a compilation of images taken at each of the test rows. The figure was used to underlie the images computed using the acoustic signals. The region denoted by the red ellipse was the identified damaged region. The figure is used to underlie the images computed using the acoustic signals. Figure 33 (c) is the visual representation of the expected results where the red region denotes the delaminated region in the structure.

\subsection{Results and Discussion}

The same post-processing was carried out on the acoustic signals from the parking garage as the lab specimen. The EIF was used in order to visualize the damages with a chosen cutoff value. The F1 score was again used as an indicator of the ability of the method to determine damage accurately and compare between variations of the post-processing method. The expected damaged locations used for analysis are visible in Figure 33(c). The expected damage locations were defined using the visual appearance of the area along with the sounding of the structure before testing using the impactor to strike the structure to discern differences in the acoustic response.

An obstacle of the method relying on acoustic signals was the environmental noise and noise intrinsic to the impactor setup. The noise in the impactor setup includes vibrational noise from the spring and the impactor itself. The noise must be de-coupled from the information of interest by separating it temporally or spectrally. The signals collected in the lab testing in 
Chapter 3 were 1200 samples long, corresponding to a time of 6 milliseconds, and accounted for the entirety of the surface wave from the concrete. It was less evident which data must be kept and processed to find meaningful results by viewing the signals from the field-testing in Figure 34 and Figure 35. Indeed, the signal-to-noise ratio (SNR) was roughly unity after the structure was excited.

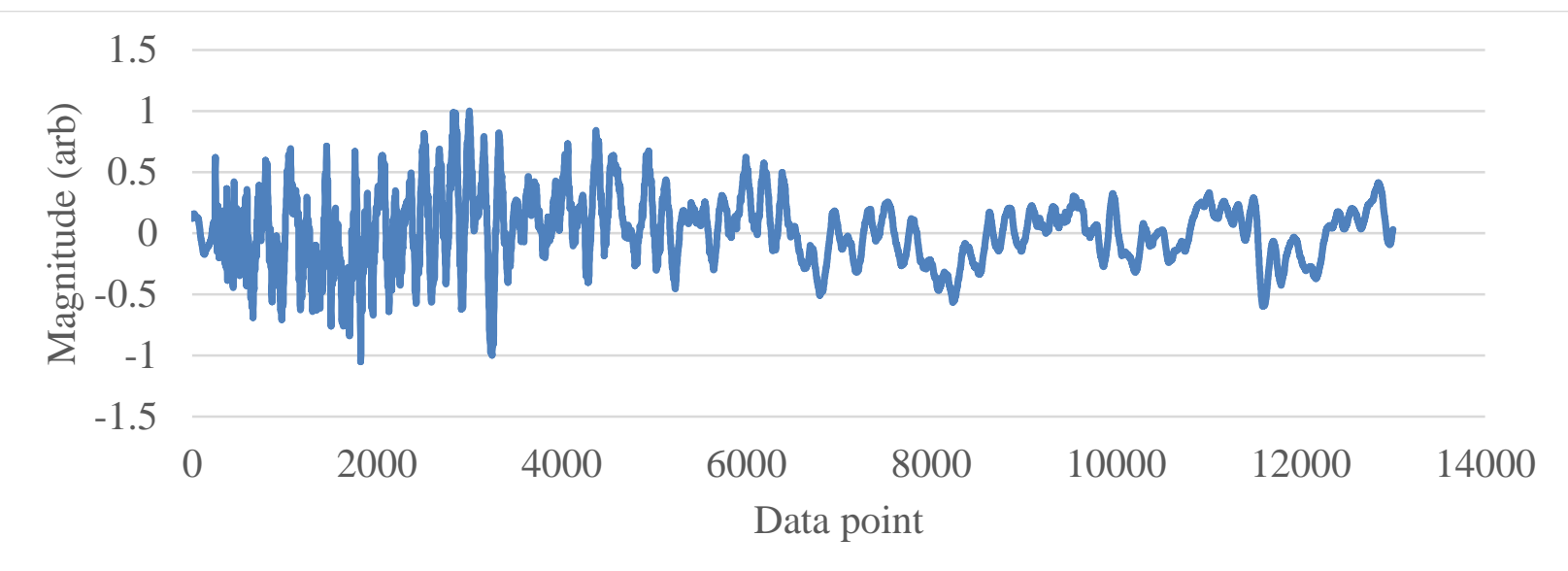

Figure 34: MIC 2 at location 1 (damaged)

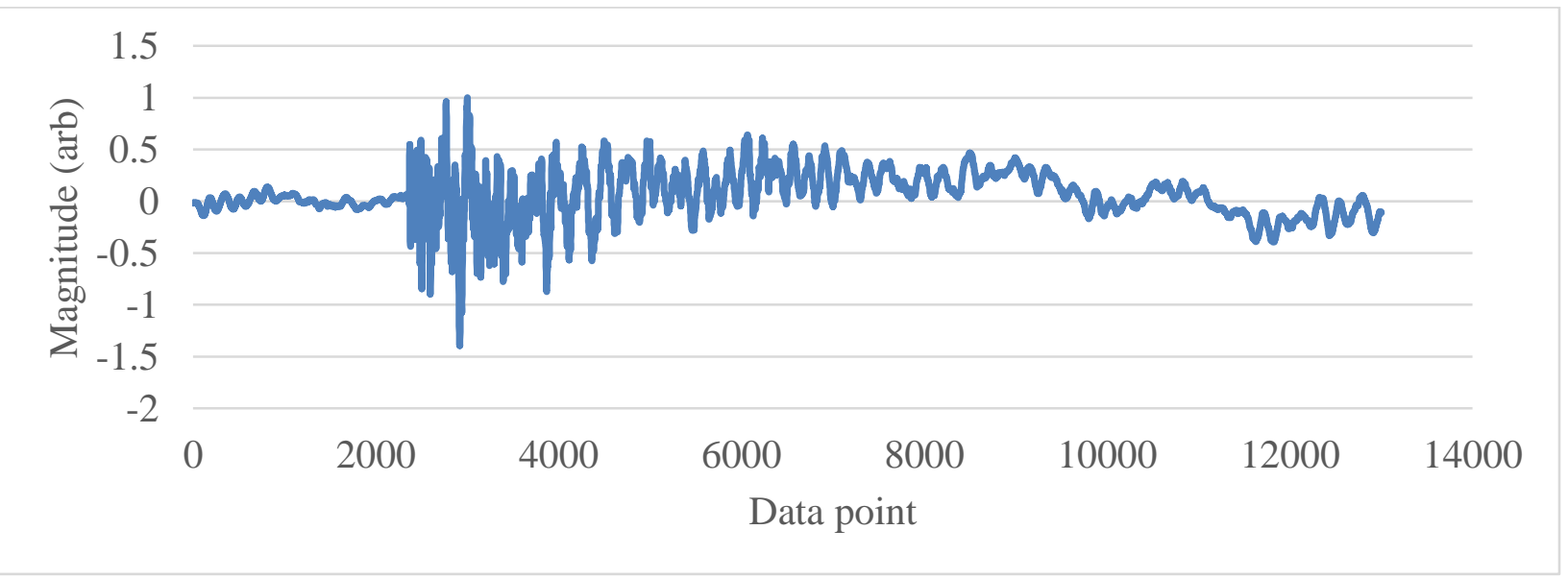

Figure 35: MIC 2 at location 11 (intact) 
Several data lengths were used to identify the region of interest in the signal. The high prevalence of noise, obvious in Figure 34 and Figure 35, made it necessary to use a substantially longer duration than the tests carried out in the lab. The spectrograms of the acquired signals were also used to determine high relative energy portions of the signal and the spectral location of the energy. Figure 36 shows the relative energy areas occurring at in the signal overall and strictly in the high frequency band.

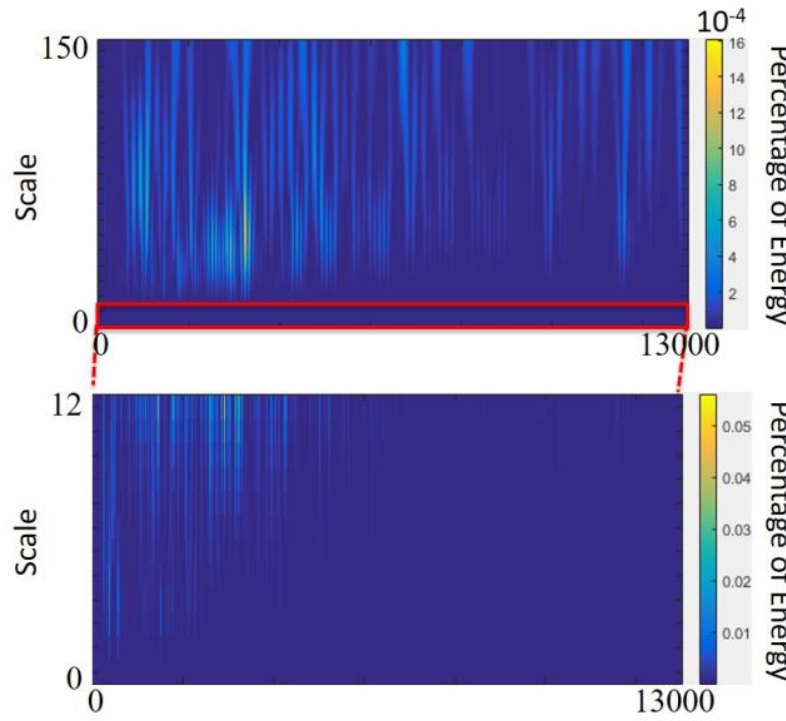

(a)

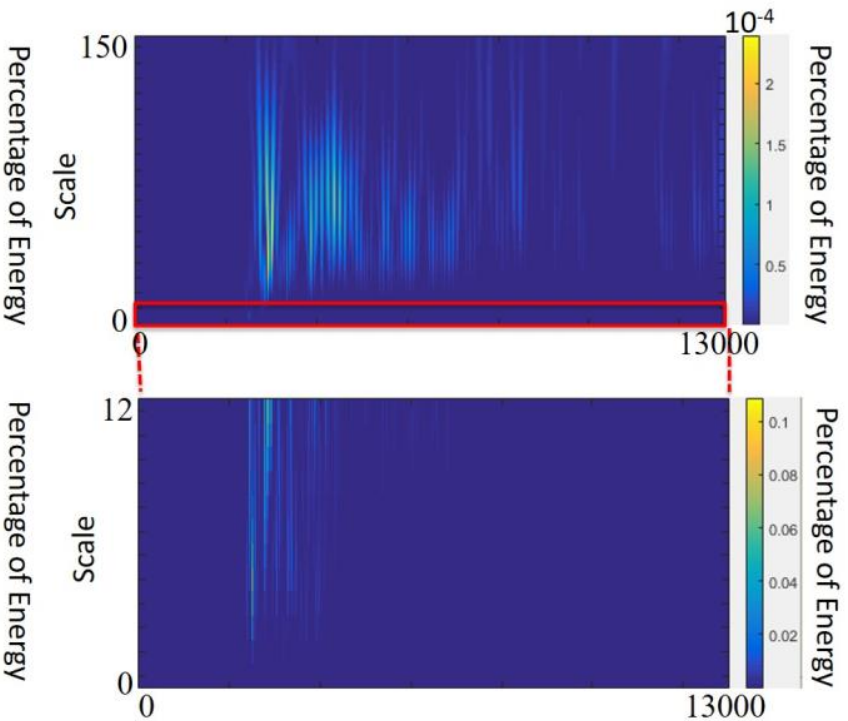

(b)

Figure 36: Percentage of energy comparison of MIC 2 at location 1 (damaged) (a) and 11 (intact) (b)

The visual representation of the damage for parking garage beam B2 is given for three different cutoff values in Figure 37-Figure 39. The trials and results using the Mexican Hat wavelet over a scale range of 1:1:300 are presented in Table 7. 


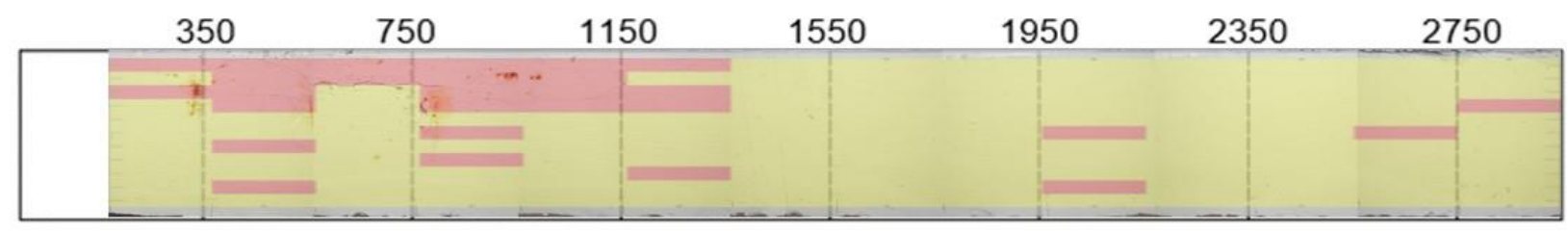

Figure 37: Parking garage delamination and cracking using Mexican Hat wavelet with cutoff of 0.12

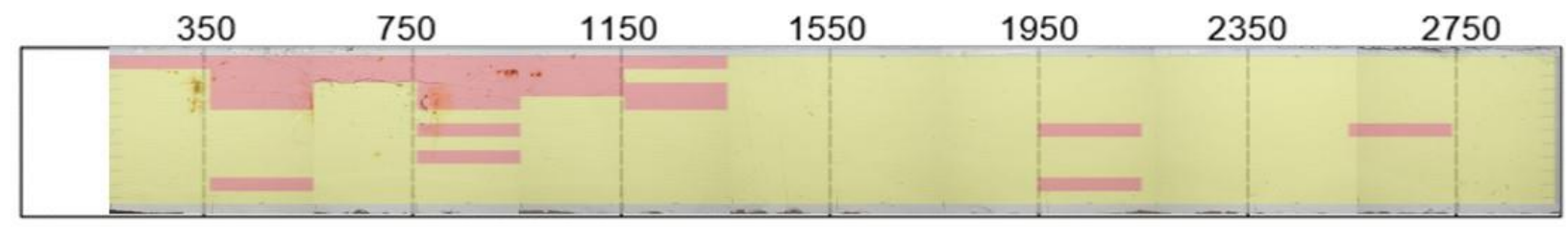

Figure 38: Parking garage delamination and cracking using Mexican Hat wavelet with cutoff of 0.15

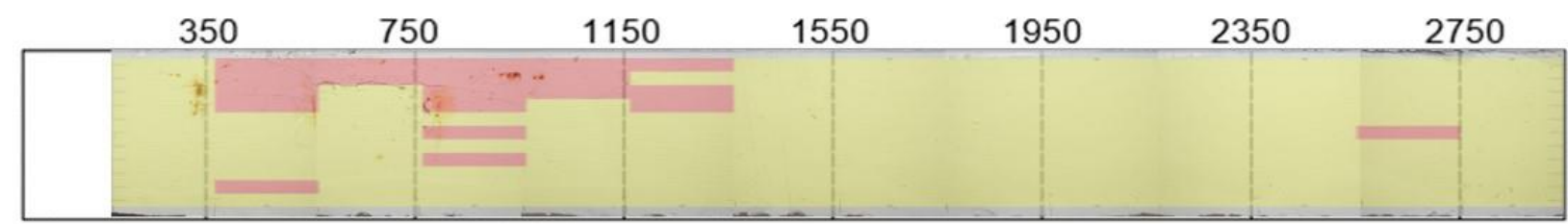

Figure 39: Parking garage delamination and cracking using Mexican Hat wavelet with cutoff of 0.18

Table 7: F1 score comparison for beam B2

\begin{tabular}{cccc}
\hline Test & $\begin{array}{c}\text { Wavelet Transform F1 } \\
\text { Score }\end{array}$ & Cutoff Value & Data Length \\
\hline B2 & 0.7077 & 0.1 & 13000 \\
\hline B2 & 0.7368 & 0.12 & 13000 \\
\hline B2 & 0.7308 & 0.15 & 13000 \\
\hline B2 & 0.72 & 0.17 & 13000 \\
\hline B2 & 0.7347 & 0.18 & 13000 \\
\hline
\end{tabular}

The method shows excellent robustness against noise. The parking garage is located at a major intersection and was subjected to noise that can be visibly seen in the data, but through the implementation of the EIFs as the damage sensitive feature, the expected damage location was readily identified. 


\section{ARTIFICIAL NEURAL NETWORK ANALYSIS}

An objective of this work was to provide a way that the user-input can be reduced in the process of identifying damaged locations in the specimens studied. One option to this end was to implement a machine learning approach in order to have a tailor-made algorithm for the particular test and the nuances that may be present. In the analysis in prior sections, cutoff values were introduced as defined by the user in order to make the distinction between intact and damaged locations. The cutoff values were chosen based on visual fit of the expected data and the quantitative performance of the results. ANNs are commonly used to analyze data and dissect inherent patterns [5-10, 28, 64]. A single-layer ANN was subsequently applied to the Test 2 and parking garage beam B2 data in an attempt to localize damage in the respective structures. Further, several parameters are manipulated in an attempt to determine the most effective algorithm.

\subsection{Artificial Neural Network Theory}

ANNs can take many forms, depending on the application. In general, ANNs use features extracted from the data in order to develop a training network that can be used to categorize subsequent feature sets. They are particularly common in the field of artificial intelligence and are becoming more prevalent in coding behind websites and apps. ANNs are used in many fields as knowledge of the general algorithm has disseminated and the strengths of the method have been repeatedly proven and improved upon. The strengths of ANNs include pattern recognition in statistical models [11].

The schematic view of the framework for the semi-autonomous method with the application of an ANN is presented as shown in Figure 1. For semi-automated testing, the aircoupled impact echo method was modified to include a testing framework of testing devices 
using an Arduino circuit for automatic movement of the sensor chassis with microphones. Once testing was carried out and the acoustic signals are gathered, post-processing using wavelet transforms is implemented and the EIF for each signal is determined from spectral bands. For automated detection of damage, the ANN was then trained using several intact and damaged locations that are identified prior to testing. Following training, the global set of EIFs was classified using the trained ANN in conjunction with a least-squared distance calculation. The results was then visualized to aid in damage detection.

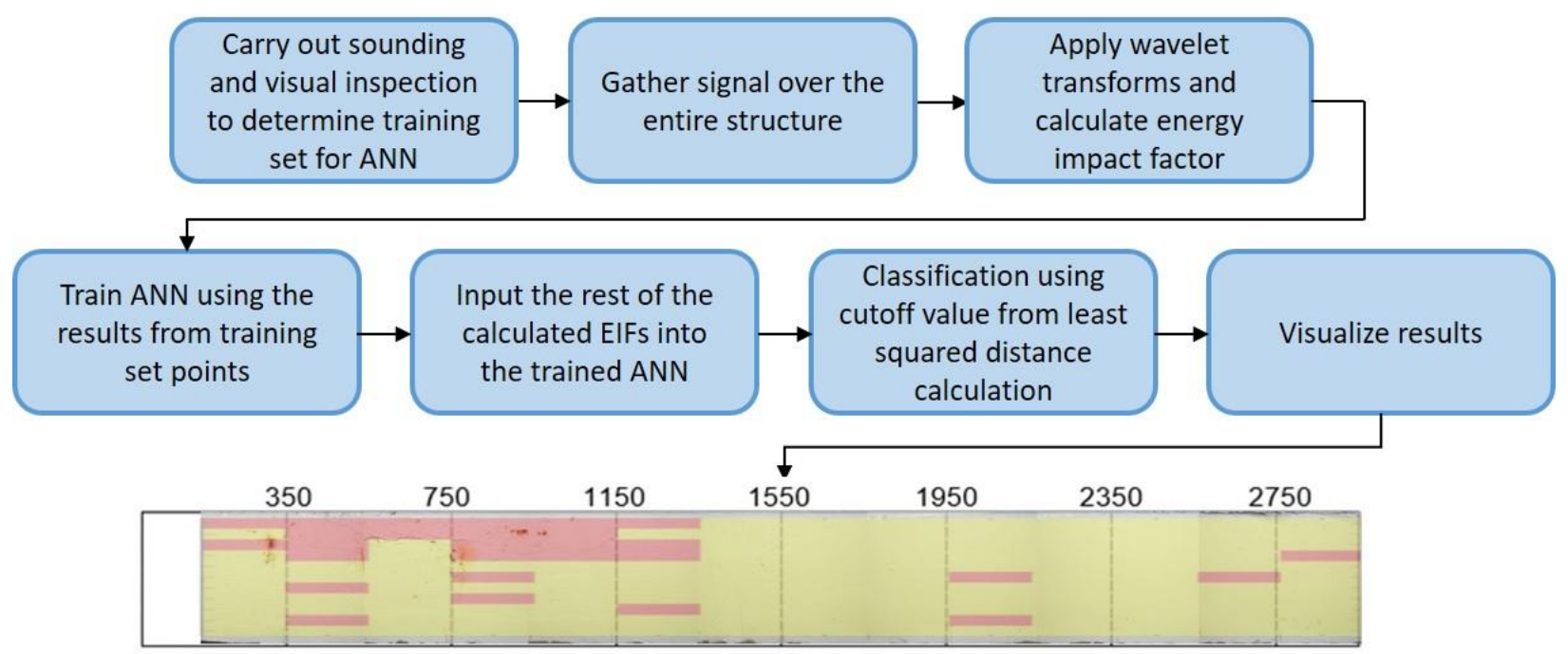

Figure 40: Schematic view of semi-automated damage detection using air-coupled impact-echo testing

The dashed lines in Figure 40 align with the measurements corresponding to impact sites. Impacts and measurements alike wer carried out every $20 \mathrm{~mm}$ in the short dimension of the beam with a spacing of $200 \mathrm{~mm}$ from the impact for both MIC 1 and MIC 2. The subsequent sections describe the process in detail. 


\subsubsection{Feature Extraction}

The use of ANNs does depend on some degree to the quality of information being used. Several features were identified as potential inputs into the ANN. Ultimately the EIF was used as the lone feature because of the ease stemming from the use a single feature and the quality of results obtained from the EIFs shown previously.

\subsubsection{Artificial Neural Networks}

The use of ANNs has lead to many different architectures [5-11]. The architecture of the ANN used in this work is discussed below.

Weights were initialized scaling a random number to the range of $0-0.5$ for both the input and hidden layer of the network. The back propagation and bias were also initialized and are set to 0 and 1 , respectively. Once the needed parameters were set, the neural network was trained using a small subset of the collected data. For this study, a feed-forward network was used with back propagation in order to incorporate the gradient of the calculated error into the training [11].

The feed-forward portion of the neural network has three main components: summation of hidden and output layer neurons from inputs, calculation of the output of the hidden and output layers, and error calculation. The summation of hidden neurons was found with Equation 29:

$$
S_{j}^{(1)}=\sum_{i=1}^{N}\left(w_{j, 1}^{(1)} x_{i}^{(1)}+w_{j, 2}^{(1)} b_{i}^{(1)}\right)
$$

Equation 29

where $w_{j, 1}^{(1)}$ and $w_{j, 2}^{(1)}$ are the weight of the hidden layer neuron for the input value and bias value, respectively, $x_{i}^{(1)}$ is the input at $i$, and $b_{i}^{(1)}$ is the bias at $i$. The value of $N$ was found from the size of the training set. The summation was carried out over all of the inputs, $i$, and applied to 
each hidden layer neuron, $j$. The superscript (1) distinguishes the hidden layer from output layer, denoted with a superscript (2) in subsequent equations.

The activation function used in neural networks can take many forms including linear, sigmoid, and softmax functions [11]. This work used the sigmoid activation function for the hidden and output layers. The sigmoid activation function was used to find the output of each neuron in each layer shown in general by Equation 30:

$$
O=1 /\left(1+e^{(-S)}\right)
$$

Equation 30

where $S$ is the summation at each neuron for the hidden or output layer given in Equation 29 and Equation 31, respectively. The error, $E_{j}$, of the output neurons compared to the expected value was calculated prior to back propagation.

The summation of the output layer at a particular neuron was found with Equation 31:

$$
S_{j}^{(2)}=w_{N+1}^{(2)} b^{(2)}+\sum_{j=1}^{N} w_{j}^{(2)} O_{j}^{(1)}
$$

where $N$ is the number of hidden layer neurons and $i$ is the particular output layer neuron. The summation of the output neurons was found with an updated $w_{i}^{(2)}$ and $b^{(2)}$ for each subsequent $S_{j}^{(2)}$. In other words, the $S_{j}^{(2)}$ value of $j=1$ was calculated with the initial weights and bias value, the weights and bias were updated using back propagation and the updated values were used for the $j=2$ output neuron.

$$
E_{i}=\left|y_{i}-O_{i}^{(2)}\right|
$$

where $y_{j}$ is the expected value of the output. 


\subsection{Back Propagation}

Back propagation allows the neural network to learn from the current iteration in order to enhance the direction of improvement of the subsequent iteration. It does this by updating the weights and bias values used in the feed-forward portion of the algorithm. Figure 41 provides details of the back propagation portion of the ANN.

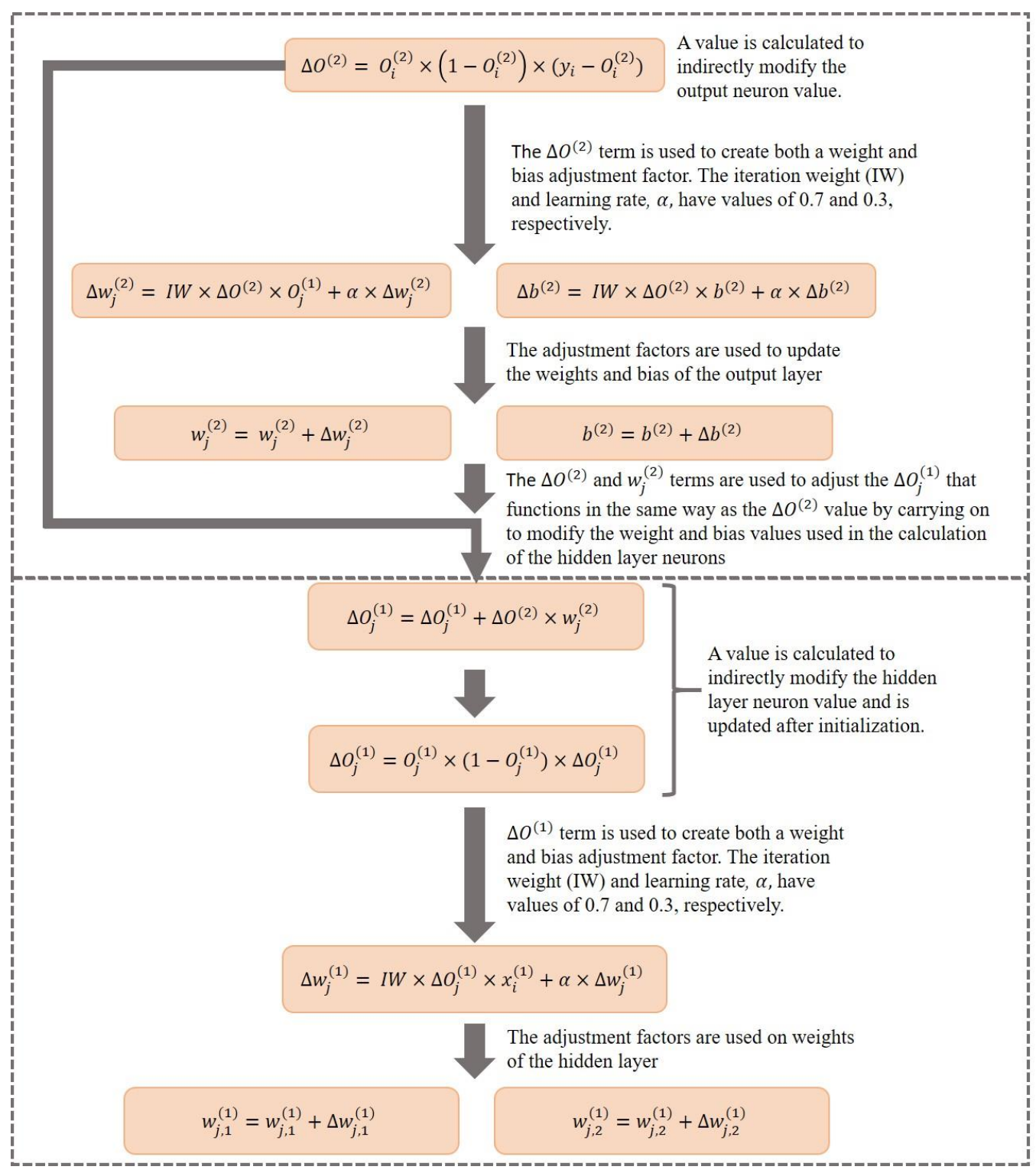

Output layer back propagation

Hidden layer back propagation

Figure 41: Back propagation algorithm for output and hidden layer 
Once the defined number of iterations was completed, the ANN was considered trained and used to classify the remaining locations on the specimen. The summation and output of the hidden layer were found using Equation 29 and Equation 30, respectively. The output of the hidden layer was then found with Equation 31 and was used in conjunction with the sigmoid function to find the outputs correlating to each input value, expressed in Equation 33:

$$
\text { Output }_{T}=1 /\left(1+e^{\left(-O_{T}^{2}\right)}\right)
$$

where $T$ is the number of test points.

\subsection{Cutoff Value Determination}

The cutoff value used was key to determining what information will signify intact and damaged locations. The cutoff value was automated based on the minimum squared distance from the cutoff value to the EIF values determined by using the wavelet transforms. This alleviated the need for a user-defined cutoff value but was predicated on the ability to separate the EIF values to a larger extent than those first found by the wavelet transforms. The ANN fosters this by definition and filters the EIFs towards 0 (intact) and 1 (damaged) poles. Figure 42 shows the squared distance of potential cutoff values (CVs) of 0.01 to 1 , increasing by 0.01 at every increment found using Equation 34; the equation for the squared distance calculation. 


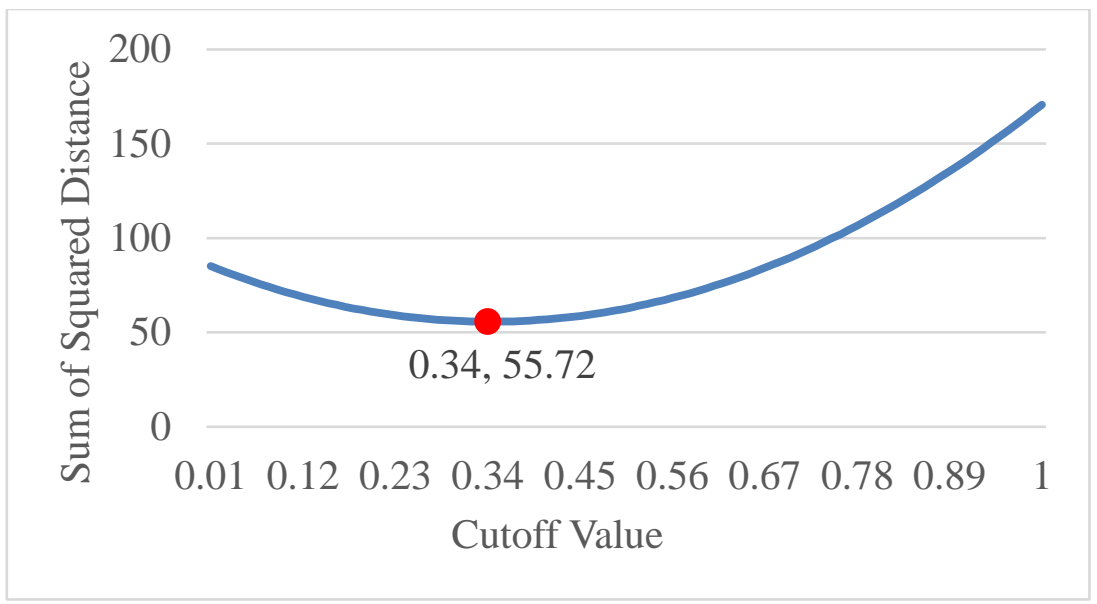

Figure 42: Cutoff value curve

$$
D=\sum_{k=1}^{T}\left(x_{k}-C V\right)^{2}
$$

Equation 34

where $x_{k}$ is the output of the ANN at location $k$ and $T$ is the total number of EIFs.

The cutoff value was used to bin the data into intact and damaged designations in order to produce a visual of the internal condition of the specimen.

\subsection{ANN Analysis Results and Discussion}

Several parameters are observed in an attempt to determine the most effective algorithm. Additionally, the iteration weight (IW), the learning rate (LR), number of features, number of neural nets, and number of output neurons are kept constant. The initial results are from data collected from Test 2 and the parking garage testing, described in Sections 3.4 and 4.2, respectively. The results from the ANN analysis are compared to the performance of the userdefined cutoff value and process.

\subsubsection{Analysis of Lab Test 2 Using an ANN}

The signals collected from the impact-echo testing were post-processed using the Mexican Hat wavelet transform using a scale range of 1-300. The EIF values were then calculated and used as 
the inputs for the ANN. The squared distance of the ANN outputs were calculated at 100 discrete values to determine the cutoff value to be used. The F1 score was then determined in order to compare the results from the use of an ANN and calculated cutoff value to those found from only the use of wavelet transforms. Table 8 outlines the fixed values throughout the various implementations of the ANN and Table 9 details the results when several parameters were adjusted to find an optimal form of the algorithm.

Table 8: Fixed values for ANN computation

\begin{tabular}{cccc}
\hline IW & LR ( $\boldsymbol{\alpha})$ & Number of Features & Number of Output Neurons \\
\hline 0.7 & 0.3 & 1 & 1 \\
\hline
\end{tabular}

Table 9: Results from preliminary parameter analysis

\begin{tabular}{ccccc}
\hline Training Set Size & $\begin{array}{c}\text { Number of Hidden } \\
\text { Neurons Per Layer }\end{array}$ & Iterations & F1 Score & Cutoff \\
\hline 10 & 20 & 100 & 0.5730 & 0.40 \\
10 & 20 & 1000 & 0.5829 & 0.34 \\
10 & 20 & 10000 & 0.5862 & 0.34 \\
10 & 40 & 100 & 0.5644 & 0.46 \\
10 & 40 & 1000 & 0.5829 & 0.34 \\
10 & 40 & 10000 & 0.5862 & 0.34 \\
20 & 40 & 100 & 0.5763 & 0.35 \\
20 & 40 & 1000 & 0.5604 & 0.36 \\
20 & 40 & 10000 & 0.5829 & 0.34 \\
20 & 100 & 100 & X (all points & - \\
& & & returned & \\
20 & 100 & damage $)$ & \\
& & 1000 & X (all points & - \\
20 & 100 & & returned & \\
34 & 40 & 10000 & 0.5683 & 0.37 \\
34 & 40 & 100 & 0.5616 & 0.59 \\
34 & 40 & 1000 & 0.5644 & 0.42 \\
& & 10000 & 0.5614 & 0.34 \\
\hline
\end{tabular}

The largest returned F1 score was found using 10 training points with 20 and 40 neurons in the hidden layer and 10000 iterations. However, comparable results were returned with several 
less computationally costly parametric arrangements. This is evidence the algorithm is sufficient with optimization within a range of parametric values.

Figure 43 and Figure 44 provide the results of damage detection with the use of the ANN in conjunction with the cutoff value calculation and only the use of the cutoff value calculation, respectively.

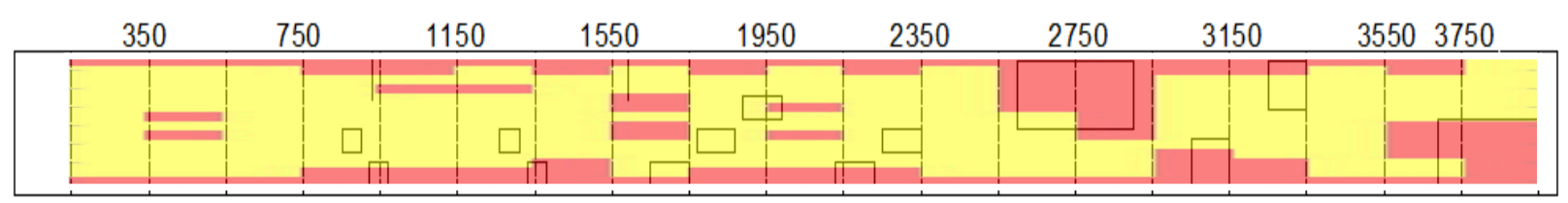

Figure 43: Damage detection with 10 training points, 40 hidden layer neurons, and 1000 iterations

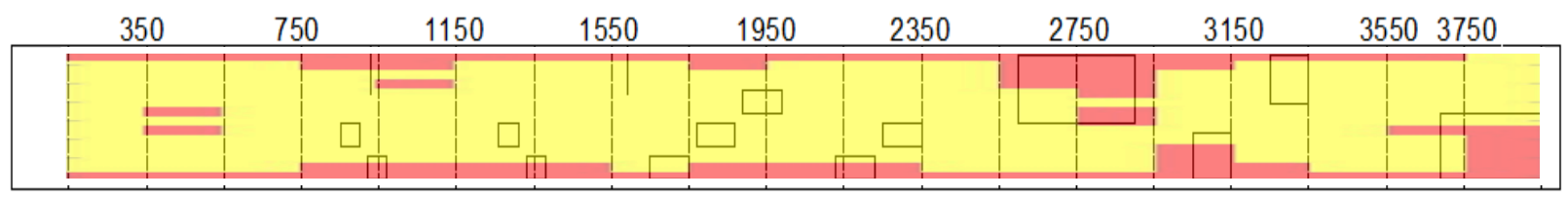

Figure 44: Damage detection with solely EIF and automated cutoff value

The F1 score of Figure 43 was 0.5829 and Figure 44 was 0.5065 , working out to a performance increase of $13.1 \%$ for the neural network approach over the direct EIF data processed with a cutoff value similarly found from the least squared distance of the data. The use of a sparse training set of only 10 data points shows excellent efficiency of the algorithm and decreases the front-end work that must be done to initialize the ANN for an SHM system.

The difference between the performance of the test with 1000 and 10000 iterations for the setup with a training data set of 10 points and 40 hidden-layer neurons was a single false positive. Therefore, while the increase in iterations provided a slightly better result, the runtime was greatly increased, with the 1000 iteration outcomes taking 13.56 seconds and the 10000 
iteration outcomes taking 134.23 seconds using MATLAB® and a Dell Precision Tower 5810 with $8 \mathrm{~GB}$ of ram.

A larger training set of 34 points was studied to analyze the effect of false positives and negatives on the ANN function and determine whether larger training sets provide improved stability in the method. Figure 45 features the image created from the ANN run with 34 training points, 40 hidden neurons, and 1000 iterations. Of the 34 test points, 12 of the expected intact points had a higher EIF value than the lowest expected damaged point and three points were false positives. The trained ANN was found to have a higher cutoff value and an increase in the number of damage locations.

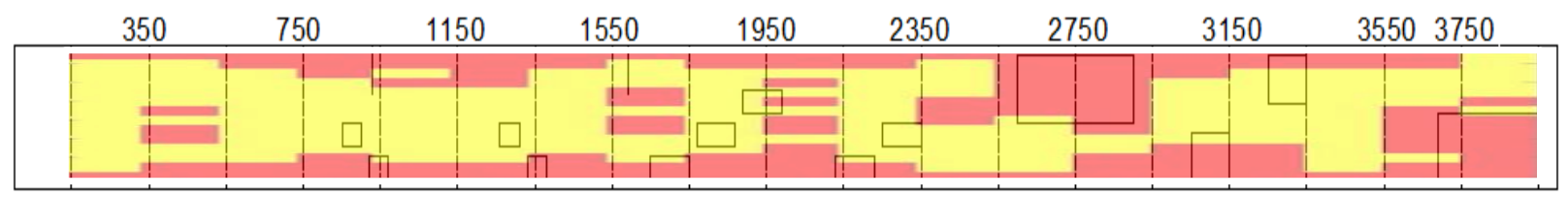

Figure 45: Damage detection with 34 training points, 40 hidden layer neurons, and 1000 Iterations

The F1 score of 0.5644 from the trial shown in Figure 45 is reasonable and shows the robustness of the ANN with respect to the size and quality of the training set.

\subsubsection{Analysis of Parking Garage Beam B2 Using an ANN}

The results from the parking garage test were also analyzed using an ANN with the same architecture as discussed previously. The constant values identified in Table 8 were held for the subsequent analysis. The cutoff value calculation used the squared distance described in Equation 34. Varying parametric values were used over multiple iterations of the analysis. Table 10 outlines the trials observed and the maximum F1 scores from each trial. 
Table 10: Results from preliminary parameter analysis from 13000 data points

\begin{tabular}{ccccc}
\hline Training Set Size & $\begin{array}{c}\text { Number of Hidden } \\
\text { Neurons Per Layer }\end{array}$ & Iterations & F1 Score & Cutoff \\
\hline 10 & 20 & 100 & 0.7368 & 0.11 \\
10 & 20 & 500 & 0.7368 & 0.16 \\
10 & 20 & 1000 & 0.7368 & 0.15 \\
20 & 20 & 100 & 0.7368 & 0.17 \\
20 & 20 & 500 & 0.7368 & 0.15 \\
20 & 20 & 1000 & 0.7368 & 0.15 \\
20 & 40 & 100 & 0.7119 & 0.16 \\
20 & 40 & 1000 & 0.7368 & 0.15 \\
36 & 40 & 100 & 0.7119 & 0.15 \\
36 & 40 & 1000 & 0.65 & 0.31 \\
\hline
\end{tabular}

The ten trials are quite consistent within the set and compare well to the maximum F1 score found when a user-defined cutoff was used, with an increase of $4.1 \%$ being observed. This value was lower than the improvement of the ANN over the squared distance in the lab specimen and can possibly be attributed to pronunciation of the delaminated region in the parking garage beam. Therefore, the damage features from the acoustic signals gathered would be expected to be much more dichotomous in nature in the parking garage.

Figure 46 and Figure 47 show the results of damage detection with the use of the ANN in conjunction with the cutoff value calculation and the use of the cutoff value calculation on solely the EIF values, respectively. The images in Figure 48 show a detailed view of the damaged location in the beam and the detected damage.

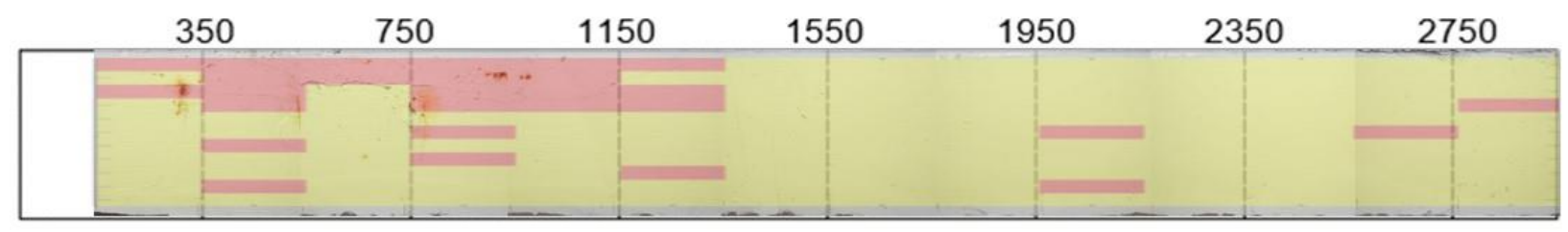

Figure 46: Damage detection with 10 training points, 20 hidden layer neurons, and 100 Iterations 


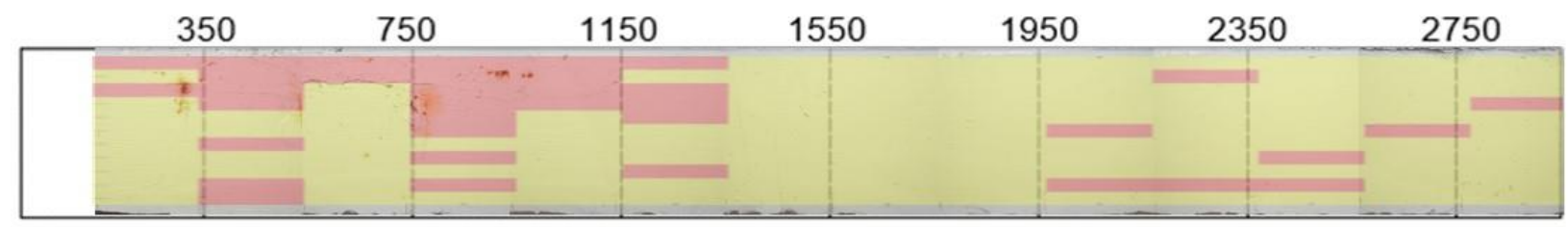

Figure 47: Damage detection with solely EIF and automated cutoff value

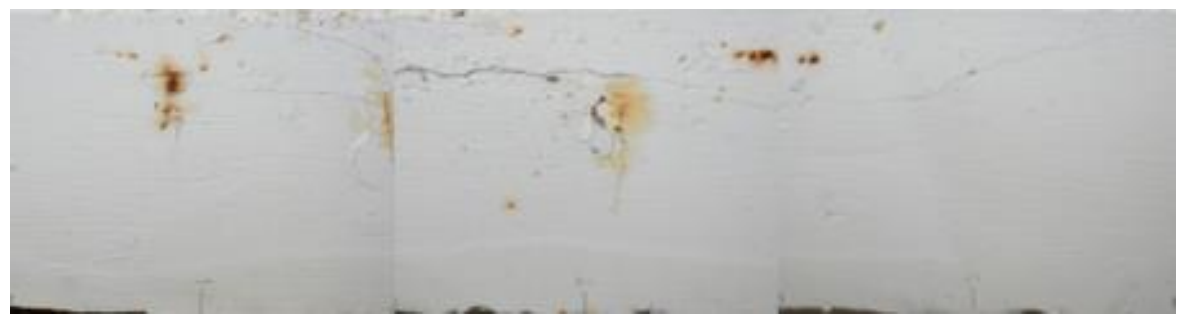

(a)

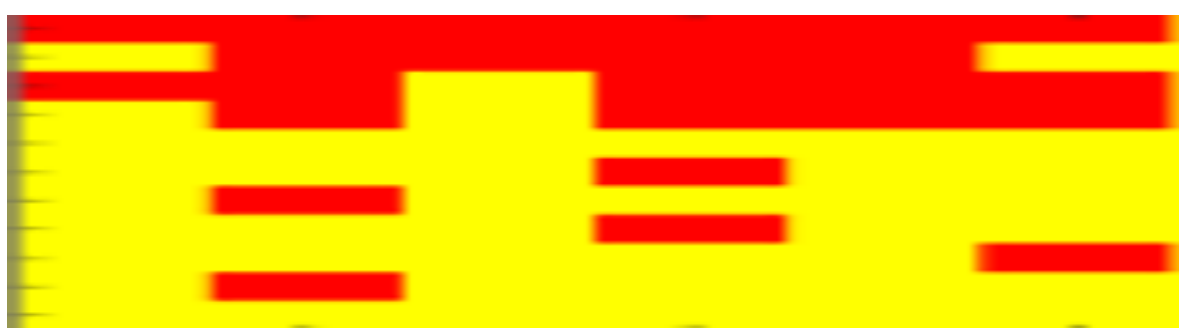

(b)

Figure 48: Comparison between damaged area of the beam (a) and the results of the damage detection analysis (b)

The 20 and 36 data point training sets contain two and five false negatives, respectively.

Both training sets contain three false positives. The results from the 36 data point training set, shown in Figure 49, shows robustness of the ANN to false positives and negatives.

\begin{tabular}{|r|r|r|r|r|r|}
\hline 350 & 750 & 1150 & 1550 & 1950 & 2350 \\
\hline \\
\hline
\end{tabular}

Figure 49: Damage detection with 34 training points, 40 hidden layer neurons, and 100 Iterations 
A total of eight of the 36 data points used in training are erroneous, based on the userdefined cutoff of 0.11 that yields the maximum result, but the results differ from the maximum of 0.7368 by only $3.3 \%$. The maximum F1 score of 0.7368 from the ANN analysis was the same result found with the user-defined cutoff value. This again shows promise in removing the need for a user-defined cutoff value.

Within the ANN trials for the parking garage beam, certain setups showed better stability in results than others. For example, the 500-iteration and 1000-iteration trial of the algorithm with 10 inputs and 20 neurons consistently produced results with an F1 score of 0.7368 . The trial with 100 iterations, on the other hand, fluctuated between F1 scores of 0.3432 and the maximum value of 0.7368 . It is possible that through adjusting the learning rate or iteration weight of the algorithm that the maximum F1 score could be found more regularly. It is important to note that the result never exceeds 0.7368 , but as it does reach it and it was taken to be the best possible result with the given data and therefore the maximum value may be used for comparison.

\section{CONCLUSION AND FUTURE WORK}

Damage detection using the frequency response of impact-echo testing was carried out on a large $\mathrm{RC}$ beam in the lab and a parking garage beam. This work proposed a wavelet transform-based damage detection method. In order to validate the performance of the proposed method, comparative studies were carried out using traditional FFT and the proposed wavelet transforms on the acoustic signals were collected from two microphones in an attempt to identify in-situ damage in the specimen. The coefficients resulting from the wavelet transforms were used to construct percentage of energy scalograms. The ratio of percentage of energy from the damage sensitive scale range and the low frequency scale range was used in order to create EIFs that 
correspond to the intact or damaged state of the RC structure.

The FFT analysis of the lab testing yielded the location of the largest damages but failed to determine the locations of the small damages or the cracks. Moving the microphones to a distance of $200 \mathrm{~mm}$ was found to be hindering to the performance of the method overall. The wavelet transform method was also unable to locate the small damages and cracks.

The overall performances of the proposed wavelet transform based damage detection method are superior to the traditional FFT based method in terms of accuracy and efficiency. The use of the Mexican Hat wavelet reduces the instances of false positives from 60 to 40, 55 to 39, and 16 to 9 when the entire structure is observed for Test 1 , the entire structure for Test 2 , and the large damages in Test 2, respectively, over the use of the FFT. The use of the Mexican Hat wavelet transform produces an increase in false positives observed for the large damages in Test 1, producing 11 compared to 10 from the FFT analysis. The intermediate damage cases with a size of $100 \times 50 \mathrm{~mm}$ were expected to be detected in at least some instances by both methods but may have moved during casting of the specimen, causing them to be unidentified by the testing. The large near-surface damages in the specimen are well identified by both the FFT and the wavelet transform using the calculated EIFs. The wavelet-based method, however, produces more accurate results with the extended sensor spacing when compared to the FFT method.

The sensor arrangement and post-processing allows more efficient testing to be carried out in the longitudinal dimension of the specimen. The transverse spacing of test points is cumbersome but could be remedied by the use of a sensor array or courser grid spacing.

The use of the ANN allows distinction between damaged and intact points that is binarized for imaging using a squared distance algorithm. The trials run in this study show that small training sets of 10 data points with 20 hidden neurons iterated 1000 times to update the 
weight and bias parameters is sufficient to create a visual of the internal condition of the beam. Further, the trained ANN showed reasonable robustness when larger training sets were used that contained false positives and negatives. The use of a training set with 34 data points with 12 values of intact locations larger than the smallest damaged location and three false positives returns an F1 score of 0.5644 compared to a result of 0.5829 when 10 training points are used and iterated 1000 times.

Semi-automation of impact-echo testing is demonstrated through the use of a NEMA 17 stepper motor controlled by an Arduino Uno R3 board and Easy Driver. The use of an ANN to classify results from the parking garage reports the same F1 score of 0.7368 as the algorithm run with the user-defined cutoff value. The ANN improves the results from the parking garage by $4.1 \%$ over the cutoff value used on the EIF values alone and provides the same result as the userdefined cutoff value method.

Future research should utilize larger sensor arrays with increased resolution and excitation frequencies in order to further utilize the wavelet transforms ability to analyze frequency with reference to temporal information and the robustness of the EIF in determining damage locations. The application of sensor arrays will further increase efficiency of testing and allow more accurate damage detection.

Further automation could be used to decrease user interaction with the test method. A cam on the impactor that draws the spring down to a constant compression for every test point is one example of an improvement that could be made. An actuator could be used as an alternative excitation source if the contact time with the structure is able to remain low. The lateral motion on the rails clamped to the beam could also be automated. Ultimately, a fully automated, mobile system would be desired to provide timely monitoring of the structure. 
As part of a long-term inspection, regiment further features, such as environmental factors, could be used in order to improve the performance of the method and prevent overly sensitive analysis, or more importantly, analysis that is not sensitive enough to the conditions of the structure. Future research should include testing on a field structure at varying temperatures to study the impact of environmental effects and to identify any need for an improvement to the robustness of the ANN. A longitudinal study would also be useful because it would allow data to be compared over time and determine the feasibility of using an ANN with data collected from different stages of the structure's life cycle.

Finally, the use of non-contact NDT should be investigated to further improve the efficiency and replicability of testing. This could be carried out in some capacity using drone mounted applications of ultrasonic sensors in conjunction with, or as an alternative to, thermal cameras. 


\section{REFERENCES}

[1] In, C., Schempp, F., Kim, J., \& Jacobs, L. J. (2014). A Fully Non-contact, Air-Coupled Ultrasonic Measurement of Surface Breaking Cracks in Concrete. Journal of Nondestructive Evaluation, 34(1). doi:10.1007/s10921-014-0272-6

[2] Ham, S., Song, H., Oelze, M. L., \& Popovics, J. S. (2017). A contactless ultrasonic surface wave approach to characterize distributed cracking damage in concrete. Ultrasonics, 75, 4657. doi:10.1016/j.ultras.2016.11.003

[3] Kim, G., In, C., Kim, J., Kurtis, K. E., \& Jacobs, L. J. (2014). Air-coupled detection of nonlinear Rayleigh surface waves in concrete-Application to microcracking detection. NDT \& E International, 67, 64-70. doi:10.1016/j.ndteint.2014.07.004

[4] Shokouhi, P., Wolf, J., \& Wiggenhauser, H. (2014). Detection of Delamination in Concrete Bridge Decks by Joint Amplitude and Phase Analysis of Ultrasonic Array Measurements. Journal of Bridge Engineering, 19(3), 04013005. doi:10.1061/(asce)be.1943-5592.0000513

[5] Stavroulakis, G. (1999). Impact-echo from a unilateral interlayer crack. LCP-BEM modelling and neural identification. Engineering Fracture Mechanics, 62(2-3), 165-184. doi:10.1016/s0013-7944(98)00107-6

[6] Xiang, Y., \& Tso, S. K. (2002). Detection and classification of flaws in concrete structure using bispectra and neural networks. Ndt \& E International, 35(1), 19-27. 
[7] Paulraj, M., Yaacob, S., Majid, M. A., Kazim, M. N., \& Krishnan, P. (2013). Structural Steel Plate Damage Detection using Non Destructive Testing, Frame Energy based Statistical Features and Artificial Neural Networks. Procedia Engineering, 53, 376-386. doi:10.1016/j.proeng.2013.02.049

[8] Legendre, S., Massicotte, D., Goyette, J., \& Bose, T. (2001). Neural classification of Lamb wave ultrasonic weld testing signals using wavelet coefficients. IEEE Transactions on Instrumentation and Measurement, 50(3), 672-678. doi:10.1109/19.930439

[9] Singh, T. J., Samanta, S., \& Chandrasekaran, M. (2011). Modeling of Ultrasonic Imaging of an Impacted Composite Domain Using the Artificial Neural Network and its Evaluation. Journal of Mechatronics and Intelligent Manufacturing, 2(3/4), 201.

[10] Mei, X., Gunaratne, M., \& Lu, J. J. (2004). Neural Network for Rapid Depth Evaluation of Shallow Cracks in Asphalt Pavements. Computer-Aided Civil and Infrastructure Engineering, 19, 223-230.

[11] Bishop, C. M. (2013). Pattern recognition and machine learning. New Delhi: Springer.

[12] Shateri, M. (2017). Acoustic Emission Monitoring of Damage Progression in Fiber Reinforced Polymer Rods (Unpublished master's thesis). University of Manitoba, Winnipeg, Canada.

[13] Hoegh, K., Kahzanovich, L., Ferraro, C., \& Clayton, D. (2015). Ultrasonic linear array validation via concrete test blocks. Doi: 10.1063/1.4914579 
[14] Behnia, Arash, Hwa Kian Chai, and Tomoki Shiotani. "Advanced Structural Health Monitoring of Concrete Structures with the Aid of Acoustic Emission." Construction and Building Materials (2014): 282-302. Print.

[15] Schickert, M., Krause, M., \& Müller, W. (2003). Ultrasonic Imaging of Concrete Elements Using Reconstruction by Synthetic Aperture Focusing Technique. Journal of Materials in Civil Engineering, 15(3), 235-246. doi:10.1061/(asce)0899-1561(2003)15:3(235)

[16] Ganguli, A., Rappaport, C. M., Abramo, D., \& Wadia-Fascetti, S. (2012). Synthetic aperture imaging for flaw detection in a concrete medium. NDT \& E International, 45(1), 79-90. doi:10.1016/j.ndteint.2011.09.004

[17] Hoegh, K., \& Khazanovich, L. (2015). Extended synthetic aperture focusing technique for ultrasonic imaging of concrete. NDT \& E International, 74, 33-42. doi:10.1016/j.ndteint.2015.05.001

[18] Beniwal, S., \& Ganguli, A. (2015a). Localized Condition Monitoring Around Rebars using Focused Ultrasonic Field and SAFT. Research in Nondestructive Evaluation,27(1), 48-67.

[19] Schickert, M. (2012). Automated ultrasonic scanning system for three-dimensional SAFT imaging of concrete elements using an electronically switched transducer array. 2012 IEEE International Ultrasonics Symposium. doi:10.1109/ultsym.2012.0011

[20] Flaherty, J. J., and Erikson, K. R., and Lund, V. M. (1970). U.S. Patent No. 3,548,642. Washington, DC: U.S. 
[21] Mohammed, T. U., \& Mahmood, A. H. (2016). Effects of maximum aggregate size on UPV of brick aggregate concrete. Ultrasonics, 69, 129-136. doi:10.1016/j.ultras.2016.04.006

[22] Hugenschmidt, J., \& Mastrangelo, R. (2006). GPR inspection of concrete bridges. Cement and Concrete Composites, 28(4), 384-392. doi:10.1016/j.cemconcomp.2006.02.016

[23] Saarenketo, T., \& Scullion, T. (2000). Road evaluation with ground penetrating radar. Journal of Applied Geophysics, 43(2-4), 119-138. doi:10.1016/s0926-9851(99)00052-х

[24] Varnavina, A. V., Khamzin, A. K., Torgashov, E. V., Sneed, L. H., Goodwin, B. T., \& Anderson, N. L. (2015). Data acquisition and processing parameters for concrete bridge deck condition assessment using ground-coupled ground penetrating radar: Some considerations. Journal of Applied Geophysics, 114, 123-133. doi:10.1016/j.jappgeo.2015.01.011

[25] Cassidy, N. J., Eddies, R., \& Dods, S. (2011). Void detection beneath reinforced concrete sections: The practical application of ground-penetrating radar and ultrasonic techniques. Journal of Applied Geophysics, 74(4), 263-276. doi:10.1016/j.jappgeo.2011.06.003

[26] Mirmiran, A., \& Wei, Y. (2001). Damage Assessment of FRP-Encased Concrete Using Ultrasonic Pulse Velocity. Journal of Engineering Mechanics, 127(2), 126-135. doi:10.1061/(asce)0733-9399(2001)127:2(126)

[27] Liu, P., \& Yeh, P. (2011). Spectral tomography of concrete structures based on impact echo depth spectra. NDT \& E International, 44(8), 692-702. doi:10.1016/j.ndteint.2010.09.013 
[28] Dworakowski, Ziemowit, Lukasz Ambrozinski, Pawel Packo, Krzysztof Dragan, and Tadeusz Stepinski. "Application of Artificial Neural Networks for Compounding Multiple Damage Indices in Lamb-wave-based Damage Detection." Structural Control and Health Monitoring Struct. Control Health Monit. (2014): 50-61. Print.

[29] Modes of Sound Wave Propagation. (n.d.). Retrieved March 15, 2017, from https://www.ndeed.org/EducationResources/CommunityCollege/Ultrasonics/Physics/modepropagation.htm

[30] Clark, M., Mccann, D., \& Forde, M. (2003). Application of infrared thermography to the non-destructive testing of concrete and masonry bridges. NDT \& E International, 36(4), 265-275. doi:10.1016/s0963-8695(02)00060-9

[31] Cha, Y., You, K., \& Choi, W. (2016). Vision-based detection of loosened bolts using the Hough transform and support vector machines. Automation in Construction, 71, 181-188. doi:10.1016/j.autcon.2016.06.008

[32] Yeum, C. M., \& Dyke, S. J. (2015). Vision-Based Automated Crack Detection for Bridge Inspection. Computer-Aided Civil and Infrastructure Engineering, 30(10), 759-770. doi:10.1111/mice. 12141

[33] Nishikawa, T., Yoshida, J., Sugiyama, T., \& Fujino, Y. (2011). Concrete Crack Detection by Multiple Sequential Image Filtering. Computer-Aided Civil and Infrastructure Engineering, 27(1), 29-47. doi:10.1111/j.1467-8667.2011.00716.x 
[34] Ziou, D., \& Tabbone, S. (1998). Edge detection techniques-an overview. Pattern Recognition and Image Analysis C/C of Raspoznavaniye Obrazov I Analiz Izobrazhenii, 8, 537-559.

[35] Cha, Y. J., Choi, W., \& Buyukozturk, O. (2017). Deep learning-based crack damage detection using convolutional neural network". Computer-Aided Civil and Infrastructure Engineering, 32(3), 2013-2014.

[36] Mutlib, Nadom Khalifa, Shahrizan Bin Baharom, Ahmed El-Shafie, and Mohd Zaki Nuawi. "Ultrasonic Health Monitoring in Structural Engineering: Buildings and Bridges." Structural Control and Health Monitoring Struct. Control Health Monit. Print.

[37] Beattie, A. (2013). Acoustic emission non-destructive testing of structures using source location techniques. doi:10.2172/1096442

[38] Berriman, J., Hutchins, D., Neild, A., Gan, T., \& Purnell, P. (2006). The application of timefrequency analysis to the air-coupled ultrasonic testing of concrete. IEEE Transactions on Ultrasonics, Ferroelectrics and Frequency Control IEEE Trans. Ultrason., Ferroelect., Freq. Contr., 53(4), 768-776.

[39] Carino, N. J. (2001). T he Impact-Echo Method: An Overview. Structures 2001.

[40] Elbatanouny, M., Larosche, A., Mazzoleni, P., Ziehl, P., Matta, F., \& Zappa, E. (2012). Identification of Cracking Mechanisms in Scaled FRP Reinforced Concrete Beams using Acoustic Emission. Exp Mech Experimental Mechanics, 69-82. 
[41] Epp, T., \& Cha, Y. J. (2016). Air-coupled impact-echo damage detection in reinforced concrete using wavelet transforms. Smart Materials and Structures, 26(2), 025018.

[42] Media, M. (n.d.). Ultrasonic Sensors. Retrieved May 03, 2017, from http://www.senscomp.com/ultrasonic-sensors/

[43] Data Presentation. (n.d.). Retrieved March 13, 2017, from https://www.ndeed.org/EducationResources/CommunityCollege/Ultrasonics/EquipmentTrans/DataPres.htm

[44] Ghiassi, B., Verstrynge, E., Lourenço, P., \& Oliveira, D. (2014). Characterization of debonding in FRP-strengthened masonry using the acoustic emission technique. Engineering Structures, 24-34.

[45] Grabowska, J., Palacz, M., \& Krawczuk, M. (2008). Damage identification by wavelet analysis. Mechanical Systems and Signal Processing, 22 (7), 1623-1635.

[46] Groschup, R., \& Grosse, C. (2015). MEMS Microphone Array Sensor for Air-Coupled Impact-Echo. Sensors, 14932-14945.

[47] Gibson, A., \& Popovics, J. S. (2005). Lamb Wave Basis for Impact-Echo Method Analysis. Journal of Engineering Mechanics, 131(4), 438-443. doi:10.1061/(asce)07339399(2005)131:4(438)

[48] Sugimoto, K., Sugimoto, T., Utagawa, N., \& Katakura, K. The Non-contact Acoustic Inspection Method for Concrete Structures using the Defect Detection Algorithm that Combined Spectrum Entropy with Vibrational Energy Ratio. 
[49] Ham, S., \& Popovics, J. (2015a). Application of contactless ultrasound toward automated inspection of concrete structures. Automation in Construction, 155-164.

[50] Ham, S., \& Popovics, J. (2015b). Application of Micro-Electro-Mechanical Sensors Contactless NDT of Concrete Structures. Sensors, 9078-9096.

[51] Henault, J., Quiertant, M., Delepine-Lesoille, S., Salin, J., Moreau, G., Taillade, F., \& Benzarti, K. (2012). Quantitative strain measurement and crack detection in RC structures using a truly distributed fiber optic sensing system. Construction and Building Materials, 37, 916-923.

[52] Hévin, G., Abraham, O., Pedersen, H., \& Campillo, M. (1998). Characterization of surface cracks with Rayleigh waves: A numerical model. NDT \& E International, 289-297.

[53] Jacobs, L., \& Owino, J. (2000). Effect of Aggregate Size on Attenuation of Rayleigh Surface Waves in Cement-Based Materials. J. Eng. Mech. Journal of Engineering Mechanics, 1124-1130.

[54] Kalamkarov, A., Saha, G., Rokkam, S., Newhook, J., \& Georgiades, A. (2005). Strain and deformation monitoring in infrastructure using embedded smart FRP reinforcements. Composites Part B: Engineering, 455-467.

[55] Kee, S., \& Zhu, J. (2009). Using air-coupled sensors to determine the depth of a surfacebreaking crack in concrete. The Journal of the Acoustical Society of America J. Acoust. Soc. Am., 1279-1279. 
[56] Staszewski, W. J. (1998). Structural and mechanical damage detection using wavelets. The Shock and Vibration Digest, 30(6), 457-472.

[57] Kim, H., \& Melhem, H. (2003). Damage detection of structures by wavelet analysis. Engineering Structures, 26(3), 347-362.

[58] Kundu, T., Ehsani, M., Maslov, K., \& Guo, D. (1999). C-scan and L-scan generated images of the concrete/GFRP composite interface. NDT \& E International, 61-69.

[59] Li, H., Huang, Y., Chen, W., Ma, M., Tao, D., \& Ou, J. (2011). Estimation and Warning of Fatigue Damage of FRP Stay Cables Based on Acoustic Emission Techniques and Fractal Theory. Computer-Aided Civil and Infrastructure Engineering, 500-512.

[60] Mirmiran, A., \& Philip, S. (2000). Comparison of acoustic emission activity in steelreinforced and FRP-reinforced concrete beams. Construction and Building Materials, 299310.

[61] Nogueira, C. L. (2011). Wavelet-based analysis of ultrasonic longitudinal and transverse pulses in cement-based materials. Cement and Concrete Research, 41(11), 1185-1195.

[62] Oh, T., \& Popovics, J. (2014). Application of Impact Resonance C-Scan Stack Images to Evaluate Bridge Deck Conditions. J. Infrastruct. Syst. Journal of Infrastructure Systems, 04014029-04014029.

[63] Zhang, H. (2003). Automatic P-Wave Arrival Detection and Picking with Multiscale Wavelet Analysis for Single-Component Recordings. Bulletin of the Seismological Society of America, 93(5), 1904-1912. doi:10.1785/0120020241 
[64] Oliveira, R., \& Marques, A. (2007). Health monitoring of FRP using acoustic emission and artificial neural networks. Computers \& Structures, 367-373.

[65] Quek, S. T., Wang, Q., Zhang, L., \& Ong, K. H. (2001). Practical issues in the detection of damage in beams using wavelets. Smart Mater. Struct. Smart Materials and Structures, 10(5), 1009-1017.

[66] Xidong, L., Ju, F., \& Ling, C. (2000). Research on the brittle fracture of FRP rods and its acoustic emission detection. 2000 IEEE Power Engineering Society Winter Meeting. Conference Proceedings (Cat. No.00CH37077).

[67] Zhu, J. (2005). Non-contact NDT of concrete structures using air-coupled sensors (Unpublished doctoral dissertation). University of Illinois at Urbana-Champaign.

[68] Zhu, J., Popovics, J., \& Schubert, F. (2004). Leaky Rayleigh and Scholte waves at the fluidsolid interface subjected to transient point loading.The Journal of the Acoustical Society of America J. Acoust. Soc. Am., 2101-2101.

[69] The Age of Public Infrastructure in Canada. (2009). Retrieved April 26, 2016, from http://www.statcan.gc.ca/pub/11-621-m/11-621-m2006035-eng.htm

[70] 2013 Report Card for American Infrastructure. (2013). Retrieved April 26, 2016, from http://www.infrastructurereportcard.org/bridges/ 
[71_Chen, J.; Wadhwa, N.; Cha, Y.J.; Durand, F.; Freeman, F.; Büyüköztürk, O. Modal identification of simple structures with high-speed video using motion magnification. $J$. Sound Vib. 2015, 345, 58-71.

[72_Cha, Y.J.; Chen, J.; Büyüköztürk, O. Motion magnification based damage detection using high speed video. In Proceedings of the 10th International Workshop on Structural Health Monitoring (IWSHM), Stanford, CA, USA, 1-3 September 2015.

[73] Qiu, Q., \& Lau, D. (2016). The Sensitivity of Acoustic-Laser Technique for Detecting the Defects in CFRP-Bonded Concrete Systems. Journal of Nondestructive Evaluation J Nondestruct Eval, 35(2).

[74] Sadri, A., \& Mirkhani, K. (2009). Wave Propagation Concrete NDT Techniques for Evaluation of Structures and Materials.

[75] Yang, Y., Dorn, C., Mancini, T., Talken, Z., Kenyon, G., Farrar, C., \& Mascareñas, D. (2017). Blind identification of full-field vibration modes from video measurements with phase-based video motion magnification. Mechanical Systems and Signal Processing, 85, $567-590$.

[76] Beniwal, S., \& Ganguli, A. (2015b). Defect detection around rebars in concrete using focused ultrasound and reverse time migration. Ultrasonics, 62, 112-125.

[77] Zhang, Y., Wei, X., Tsai, Y., Zhu, J., Fetrat, F. A., \& Gucunski, N. (2012). Multisensor data fusion for impact-echo testing of concrete structures. Smart Mater. Struct. Smart Materials and Structures, 21(7), 075021. 
[78] ASTM C469 / C469M-14, Standard Test Method for Static Modulus of Elasticity and Poisson's Ratio of Concrete in Compression, ASTM International, West Conshohocken, PA, 2014.

[79] ASTM C597-09, Standard Test Method for Pulse Velocity Through Concrete, ASTM International, West Conshohocken, PA, 2009.

[80] Schmalz, B. (n.d.). EasyDriver. Retrieved May 31, 2017, from http://www.schmalzhaus.com/EasyDriver/Examples/EasyDriverExamples.html

[81] Shateri, M., Ghaib, M., Svecova, D., \& Thomson, D. (2017). On acoustic emission for damage detection and failure prediction in fiber reinforced polymer rods using pattern recognition analysis. Smart Materials and Structures, 26(6), 065023. doi:10.1088/1361$665 x / a a 6 e 43$ 


\section{APPENDIX A: WAVELET COEFFICIENT CALCULATIONS}


Table 11: Continuous wavelet transforms coefficient calculations using linear integration

\begin{tabular}{ccc}
\hline Location & Calculation & Coefficient \\
\hline 1 & $\frac{(1 \times 2)+(0 \times 1)}{2}+\frac{(0 \times 1)+(-1 \times 0)}{2}$ & 1 \\
2 & $\frac{(1 \times 1)+(0 \times 0)}{2}+\frac{(0 \times 0)+(-1 \times 2)}{2}$ & -0.5 \\
3 & $\frac{(1 \times 0)+(0 \times 2)}{2}+\frac{(0 \times 2)+(-1 \times 3)}{2}$ & -1.5 \\
4 & $\frac{(1 \times 2)+(0 \times 3)}{2}+\frac{(0 \times 3)+(-1 \times-1)}{2}$ & 1.5 \\
5 & $\frac{(1 \times 3)+(0 \times-1)}{2}$ & 1.5 \\
6 & $\frac{(1 \times-1)+0}{2}$ & -0.5 \\
\hline
\end{tabular}

Table 12: Discrete wavelet transforms

\begin{tabular}{ccc}
\hline Location & Equation & Coefficient \\
\hline 1 & $\frac{[(1 \times 2)+(1 \times 0)]}{2}+\frac{[(0 \times 0)+(-1 \times 2)]}{2}$ & 0 \\
2 & $\frac{[(1 \times 0)+(0 \times 2)]}{2}+\frac{[(0 \times 3)+(-1 \times-1)]}{2}$ & 0.5 \\
3 & $\frac{[(1 \times 3)+(0 \times-1)]}{2}+\frac{[(0 \times-2)+(-1 \times 1)]}{2}$ & 1 \\
4 & $\frac{[(1 \times-2)+(0 \times 1)]}{2}$ & -1 \\
\hline
\end{tabular}

